\title{
Morita-Baylis-Hillman Reaction of $\alpha, \beta$-Unsaturated Ketones with Allylic Acetates by the Combination of Transition-Metal Catalysis and Organomediation
}

\author{
Ya-Qiong Li, ${ }^{\text {a }}$ Hai-Jun Wang, ${ }^{\text {b }}$ and Zhi-Zhen Huang*, \\ ${ }^{\mathrm{a}}$ Department of Chemistry, Zhejiang University, Hangzhou 310028, China \\ ${ }^{\mathrm{b}}$ College of Pharmaceutical Sciences, Qiqihar Medical University, Qiqihar 161006, P. \\ R. China
}

Supporting Information

Contents

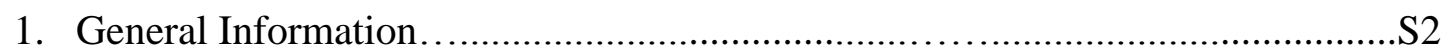

2. Optimization of the MBH Reaction Conditions...........................................S2

3. ${ }^{1} \mathrm{H}$ NMR, ${ }^{13} \mathrm{C}$ NMR, MS and HR-MS Spectra of $\alpha$-Allylated Products 3...........S4 


\section{General Information}

Unless otherwise indicated, all reagents were purchased from commercial distributors and used without further purification. ${ }^{1} \mathrm{H}$ NMR and ${ }^{13} \mathrm{C}$ NMR were recorded at $400 \mathrm{MHz}$ and $100 \mathrm{MHz}$ respectively, using tetramethylsilane as an internal reference. Mass spectroscopy data were collected on HRMS-EI, EI-MS and ESI-MS instrument. Column chromatography was performed over silica gel 200-300.

\section{Optimization of the MBH Reaction Conditions}
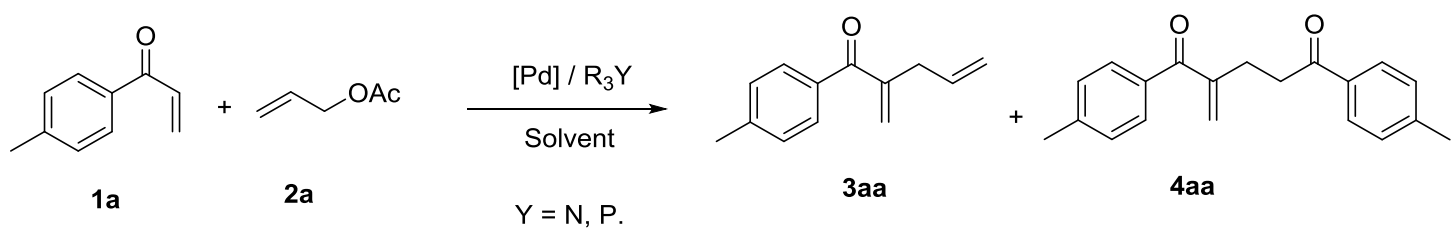

Table S1. Screening of Reaction Conditions. ${ }^{[a]}$

\begin{tabular}{|c|c|c|c|c|c|c|}
\hline \multirow{2}{*}{ Entry } & \multirow{2}{*}[\mathrm{Pd}]{} & \multirow{2}{*}{$\mathrm{R}_{3} \mathrm{Y}$} & \multirow{2}{*}{ Additive } & \multirow{2}{*}{ Solvent } & \multicolumn{2}{|c|}{ Yield $(\%)^{[\mathrm{b}]}$} \\
\hline & & & & & $3 \mathbf{a a}$ & $4 \mathbf{a a}$ \\
\hline 1 & $\mathrm{Pd}\left(\mathrm{PPh}_{3}\right)_{4}$ & $\mathrm{P}(n-\mathrm{Bu})_{3}$ & - & dioxane & 20 & 78 \\
\hline 2 & $\mathrm{Pd}(\mathrm{dba})_{2}$ & $\mathrm{P}(n-\mathrm{Bu})_{3}$ & - & dioxane & 11 & 82 \\
\hline 3 & $\mathrm{Pd}_{2}(\mathrm{dba})_{3} \cdot \mathrm{CHCl}_{3}$ & $\mathrm{P}(n-\mathrm{Bu})_{3}$ & - & dioxane & 14 & 77 \\
\hline 4 & $\operatorname{Pd}(\mathrm{dppe})_{2}$ & $\mathrm{P}(n-\mathrm{Bu})_{3}$ & - & dioxane & - & 89 \\
\hline 5 & $\mathrm{Pd}(\mathrm{OAc})_{2}$ & $\mathrm{P}(n-\mathrm{Bu})_{3}$ & - & dioxane & 9 & 82 \\
\hline 6 & $\mathrm{PdCl}_{2}$ & $\mathrm{P}(n-\mathrm{Bu})_{3}$ & - & dioxane & - & 79 \\
\hline 7 & $\mathrm{Pd}\left(\mathrm{PPh}_{3}\right)_{4}$ & $\mathrm{P}(t-\mathrm{Bu})_{3}$ & - & dioxane & - & 21 \\
\hline 8 & $\mathrm{Pd}\left(\mathrm{PPh}_{3}\right)_{4}$ & $\mathrm{P}(\text { Cyhex })_{3}$ & - & dioxane & trace & 23 \\
\hline 9 & $\mathrm{Pd}\left(\mathrm{PPh}_{3}\right)_{4}$ & $\mathrm{PPh}_{3}$ & - & dioxane & - & 13 \\
\hline 10 & $\mathrm{Pd}\left(\mathrm{PPh}_{3}\right)_{4}$ & $\mathrm{DABCO}$ & - & dioxane & - & 10 \\
\hline 11 & $\mathrm{Pd}\left(\mathrm{PPh}_{3}\right)_{4}$ & $\mathrm{DBU}$ & - & dioxane & - & 19 \\
\hline 12 & $\mathrm{Pd}\left(\mathrm{PPh}_{3}\right)_{4}$ & TEA & - & dioxane & - & 7 \\
\hline 13 & $\mathrm{Pd}\left(\mathrm{PPh}_{3}\right)_{4}$ & $\mathrm{P}(n-\mathrm{Bu})_{3}$ & $\mathrm{BzOH}$ & dioxane & 50 & 45 \\
\hline 14 & $\operatorname{Pd}(\mathrm{dba})_{2}$ & $\mathrm{P}(n-\mathrm{Bu})_{3}$ & $\mathrm{BzOH}$ & dioxane & 39 & 51 \\
\hline 15 & $\mathrm{Pd}_{2}(\mathrm{dba})_{3} \cdot \mathrm{CHCl}_{3}$ & $\mathrm{P}(n-\mathrm{Bu})_{3}$ & $\mathrm{BzOH}$ & dioxane & 43 & 50 \\
\hline 16 & $\operatorname{Pd}(\mathrm{dppe})_{2}$ & $\mathrm{P}(n-\mathrm{Bu})_{3}$ & $\mathrm{BzOH}$ & dioxane & - & 69 \\
\hline 17 & $\mathrm{Pd}(\mathrm{OAc})_{2}$ & $\mathrm{P}(n-\mathrm{Bu})_{3}$ & $\mathrm{BzOH}$ & dioxane & 45 & 53 \\
\hline 18 & $\mathrm{PdCl}_{2}$ & $\mathrm{P}(n-\mathrm{Bu})_{3}$ & $\mathrm{BzOH}$ & dioxane & - & 66 \\
\hline 19 & $\mathrm{Pd}\left(\mathrm{PPh}_{3}\right)_{4}$ & $\mathrm{P}(t-\mathrm{Bu})_{3}$ & $\mathrm{BzOH}$ & dioxane & - & trace \\
\hline 20 & $\mathrm{Pd}\left(\mathrm{PPh}_{3}\right)_{4}$ & $\mathrm{P}(\text { Cyhex })_{3}$ & $\mathrm{BzOH}$ & dioxane & trace & trace \\
\hline
\end{tabular}




\begin{tabular}{|c|c|c|c|c|c|c|}
\hline 21 & $\mathrm{Pd}\left(\mathrm{PPh}_{3}\right)_{4}$ & $\mathrm{PPh}_{3}$ & $\mathrm{BzOH}$ & dioxane & - & trace \\
\hline 22 & $\mathrm{Pd}\left(\mathrm{PPh}_{3}\right)_{4}$ & $\mathrm{P}(n-\mathrm{Bu})_{3}$ & PivOH & dioxane & 47 & 51 \\
\hline 23 & $\mathrm{Pd}\left(\mathrm{PPh}_{3}\right)_{4}$ & $\mathrm{P}(n-\mathrm{Bu})_{3}$ & $p$-TSA & dioxane & - & - \\
\hline 24 & $\mathrm{Pd}\left(\mathrm{PPh}_{3}\right)_{4}$ & $\mathrm{P}(n-\mathrm{Bu})_{3}$ & $\mathrm{CF}_{3} \mathrm{CO}_{2} \mathrm{H}$ & dioxane & - & - \\
\hline 25 & $\operatorname{Pd}\left(\mathrm{PPh}_{3}\right)_{4}$ & $\mathrm{P}(n-\mathrm{Bu})_{3}$ & $\mathrm{AcOH}$ & dioxane & 59 & 40 \\
\hline $26^{[\mathrm{c}]}$ & $\mathrm{Pd}\left(\mathrm{PPh}_{3}\right)_{4}$ & $\mathrm{P}(n-\mathrm{Bu})_{3}$ & $\mathrm{AcOH}$ & dioxane & 68 & 29 \\
\hline $27^{[\mathrm{d}]}$ & $\mathrm{Pd}\left(\mathrm{PPh}_{3}\right)_{4}$ & $\mathrm{P}(n-\mathrm{Bu})_{3}$ & $\mathrm{AcOH}$ & dioxane & $82(37)^{[\mathrm{g}]}$ & 12 \\
\hline $28^{[\mathrm{d}, \mathrm{e}]}$ & $\mathrm{Pd}\left(\mathrm{PPh}_{3}\right)_{4}$ & $\mathrm{P}(n-\mathrm{Bu})_{3}$ & $\mathrm{AcOH}$ & dioxane & 58 & 11 \\
\hline $29^{[\mathrm{d}, \mathrm{f}]}$ & $\mathrm{Pd}\left(\mathrm{PPh}_{3}\right)_{4}$ & $\mathrm{P}(n-\mathrm{Bu})_{3}$ & $\mathrm{AcOH}$ & dioxane & 73 & 26 \\
\hline $30^{[\mathrm{d}]}$ & $\mathrm{Pd}\left(\mathrm{PPh}_{3}\right)_{4}$ & $\mathrm{P}(n-\mathrm{Bu})_{3}$ & $\mathrm{AcOH}$ & toluene & 60 & 31 \\
\hline $31^{[\mathrm{d}]}$ & $\mathrm{Pd}\left(\mathrm{PPh}_{3}\right)_{4}$ & $\mathrm{P}(n-\mathrm{Bu})_{3}$ & $\mathrm{AcOH}$ & DME & 74 & 16 \\
\hline $32^{[\mathrm{d}]}$ & $\mathrm{Pd}\left(\mathrm{PPh}_{3}\right)_{4}$ & $\mathrm{P}(n-\mathrm{Bu})_{3}$ & $\mathrm{AcOH}$ & $\mathrm{THF}$ & 46 & 39 \\
\hline $33^{[\mathrm{d}]}$ & $\mathrm{Pd}\left(\mathrm{PPh}_{3}\right)_{4}$ & $\mathrm{P}(n-\mathrm{Bu})_{3}$ & $\mathrm{AcOH}$ & $\mathrm{CH}_{3} \mathrm{CN}$ & - & - \\
\hline $34^{[\mathrm{d}]}$ & $\mathrm{Pd}\left(\mathrm{PPh}_{3}\right)_{4}$ & $\mathrm{P}(n-\mathrm{Bu})_{3}$ & $\mathrm{AcOH}$ & DMSO & - & - \\
\hline $35^{[\mathrm{d}, \mathrm{h}]}$ & $\mathrm{Pd}\left(\mathrm{PPh}_{3}\right)_{4}$ & $\mathrm{P}(n-\mathrm{Bu})_{3}$ & $\mathrm{AcOH}$ & dioxane & 51 & 43 \\
\hline
\end{tabular}

[a] The mixture of 1a $(0.1 \mathrm{mmol}), \mathbf{2 a}(0.3 \mathrm{mmol})$, catalyst $(10 \mathrm{~mol} \%)$, phosphine $(100 \mathrm{~mol} \%)$, and additive (1.0 eq) was stirred in solvent $(2 \mathrm{~mL})$ at $60{ }^{\circ} \mathrm{C}$ for $24 \mathrm{~h}$ under $\mathrm{N}_{2}(1 \mathrm{~atm})$. [b] Isolated yield. [c] 1.5 eq AcOH. [d] 2.0 eq AcOH. [e] At $50{ }^{\circ} \mathrm{C}$. [f] At $70{ }^{\circ} \mathrm{C}$. [g] 50 mol \% phosphine. [h] $5 \mathrm{~mol} \% \mathrm{Pd}\left(\mathrm{PPh}_{3}\right)_{4}$. 


\section{3. ${ }^{1} \mathrm{H}$ NMR, ${ }^{13} \mathrm{C}$ NMR, MS and HR-MS Spectra of $\alpha$-Allylated Products}

${ }^{1} \mathrm{H}$ NMR Spectrum of 2-Methylene-1-(4-tolyl)pent-4-en-1-one 3aa

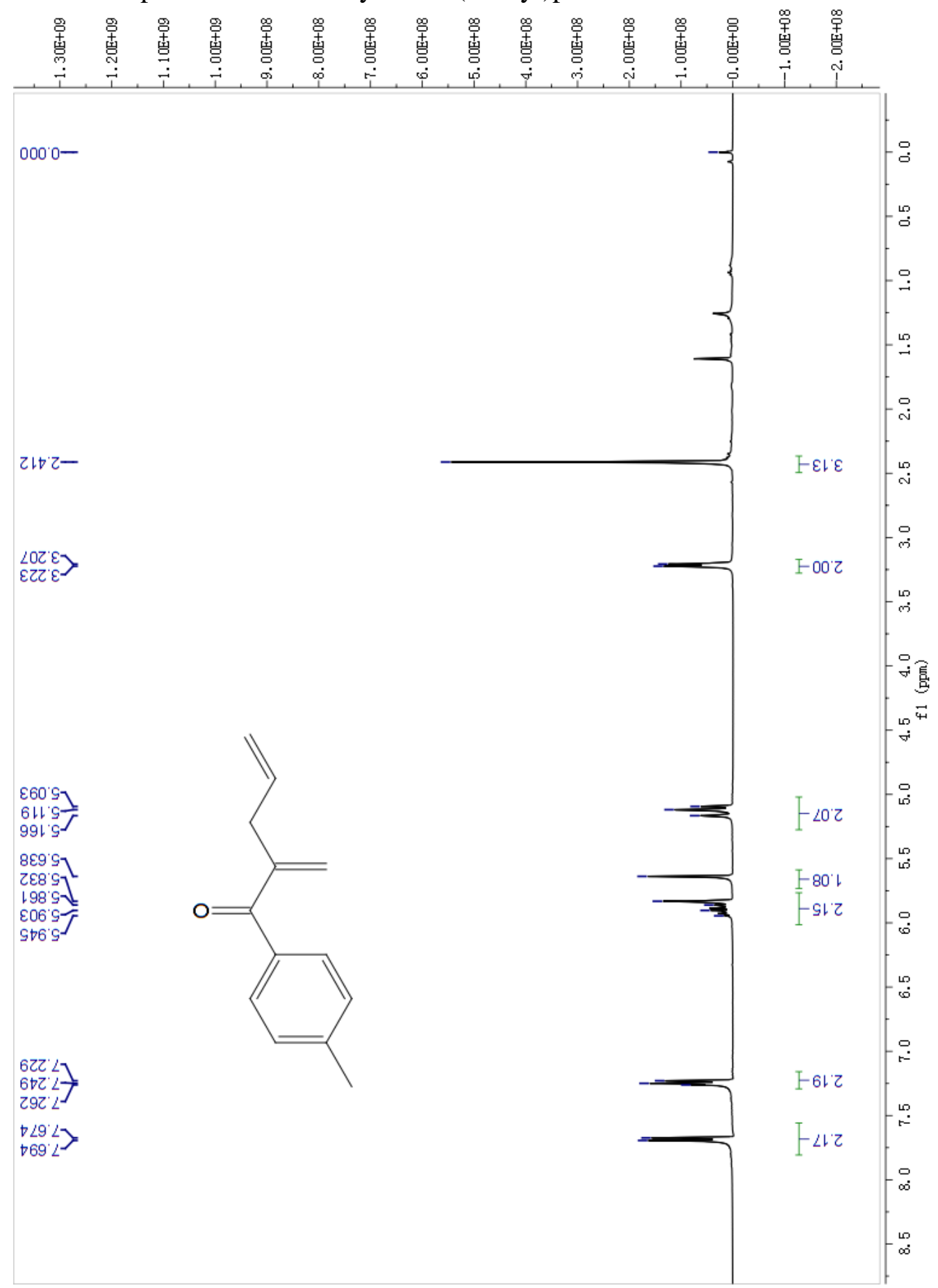


${ }^{13}$ C NMR Spectrum of 2-Methylene-1-(4-tolyl)pent-4-en-1-one 3aa

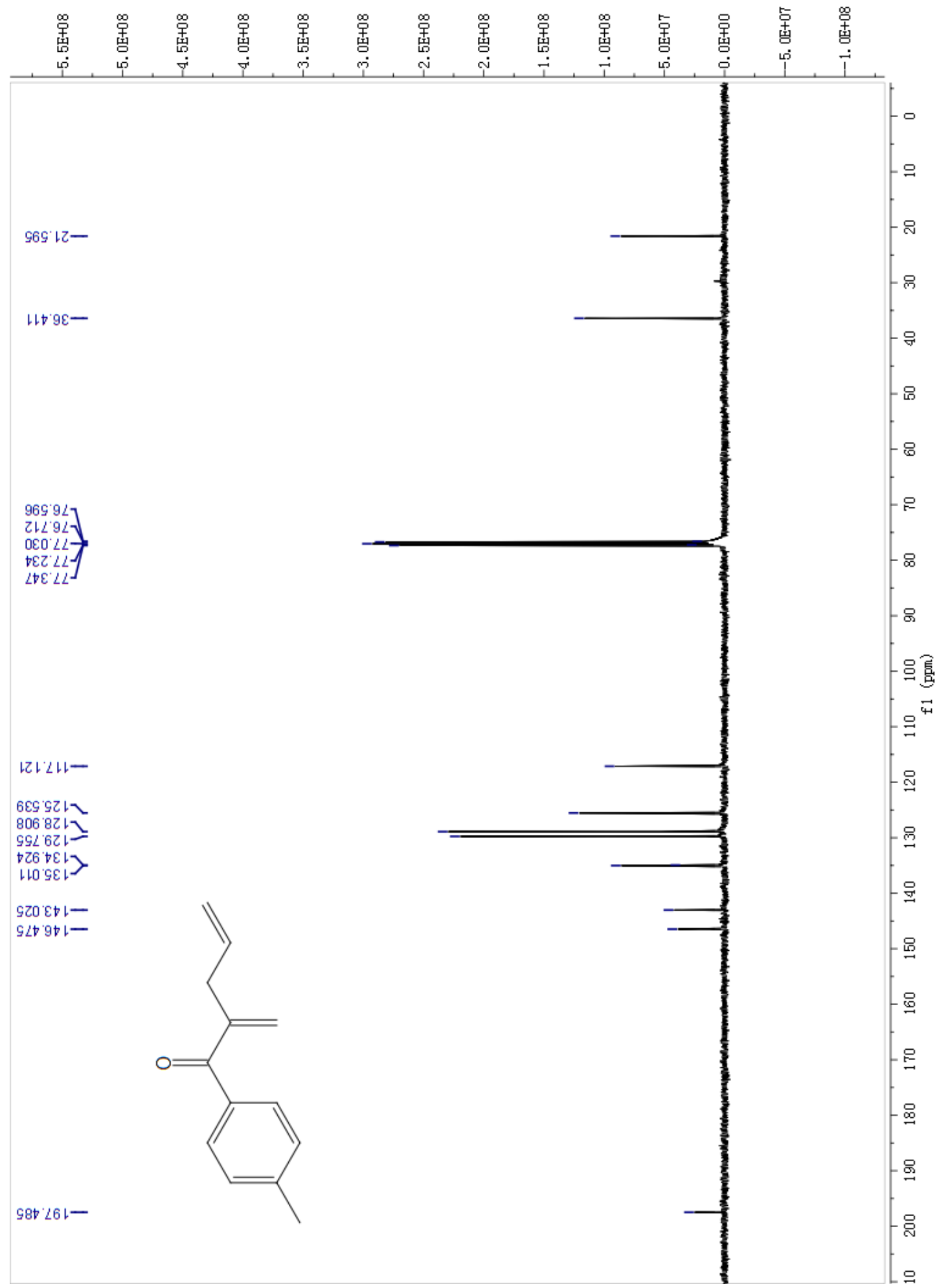


HR-MS Spectrum of 2-Methylene-1-(4-tolyl)pent-4-en-1-one 3aa

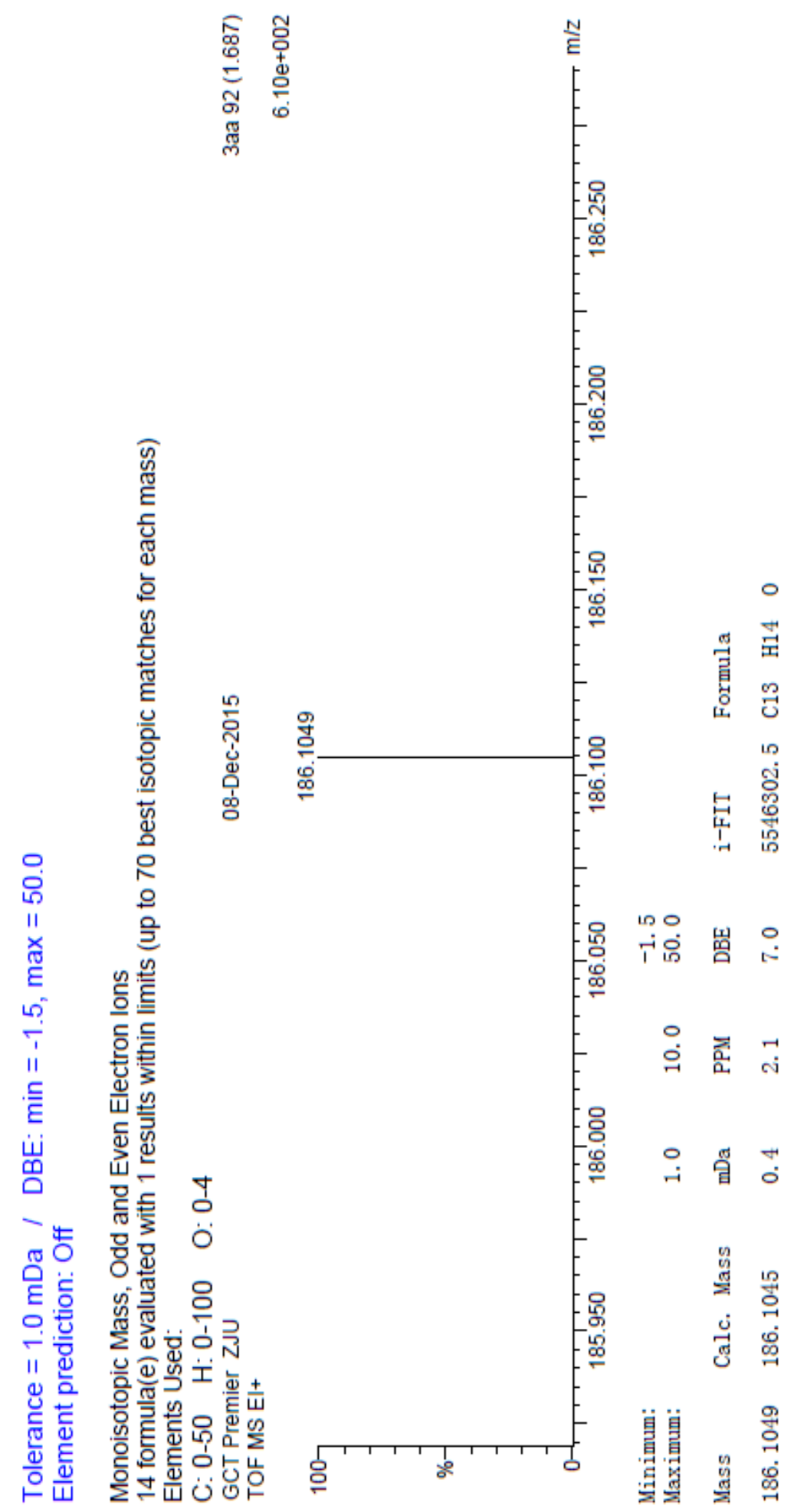


${ }^{1} \mathrm{H}$ NMR Spectrum of 2-Methylene-1-(2-tolyl)pent-4-en-1-one 3ba

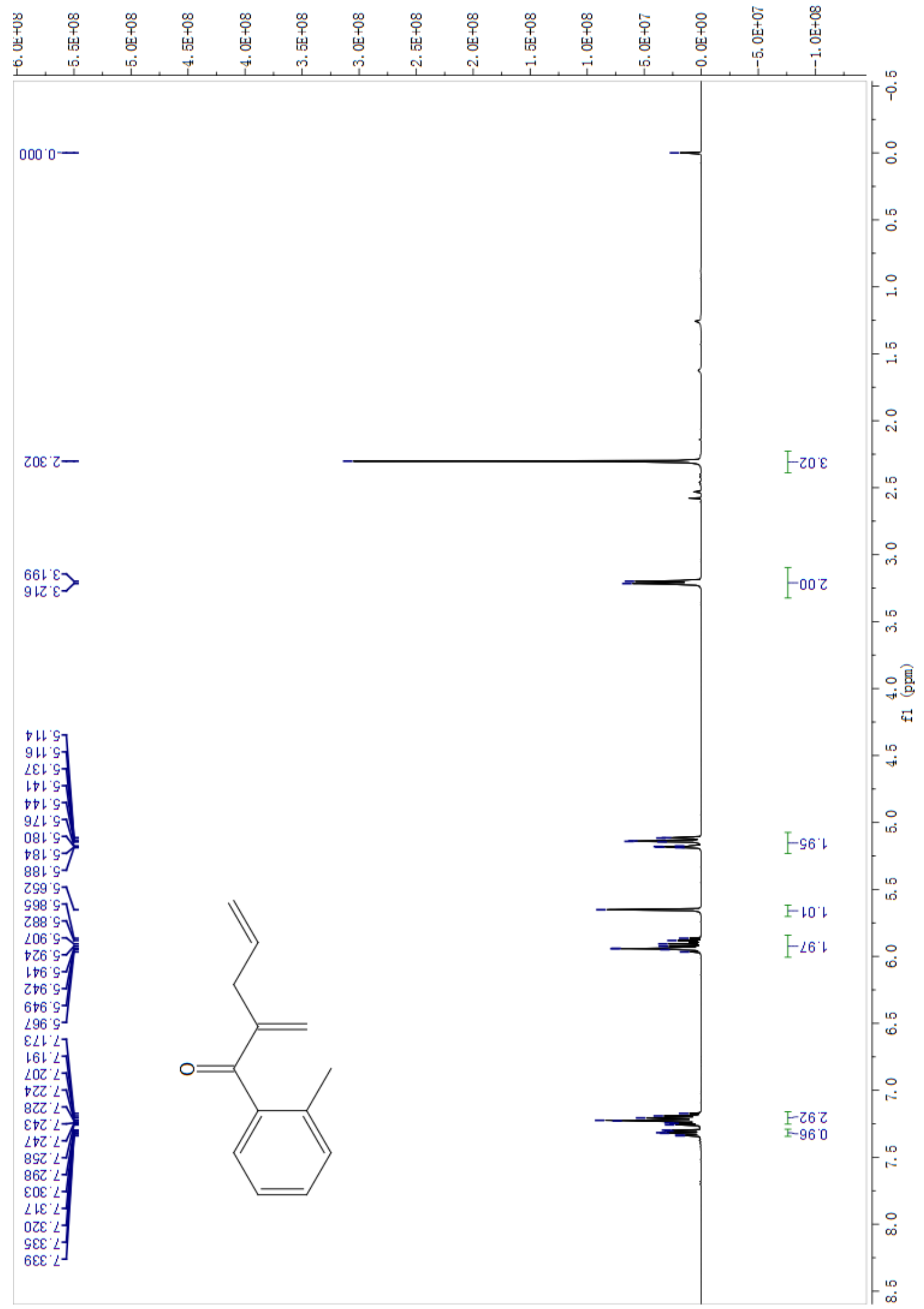


${ }^{13} \mathrm{C}$ NMR Spectrum of 2-Methylene-1-(2-tolyl)pent-4-en-1-one 3ba

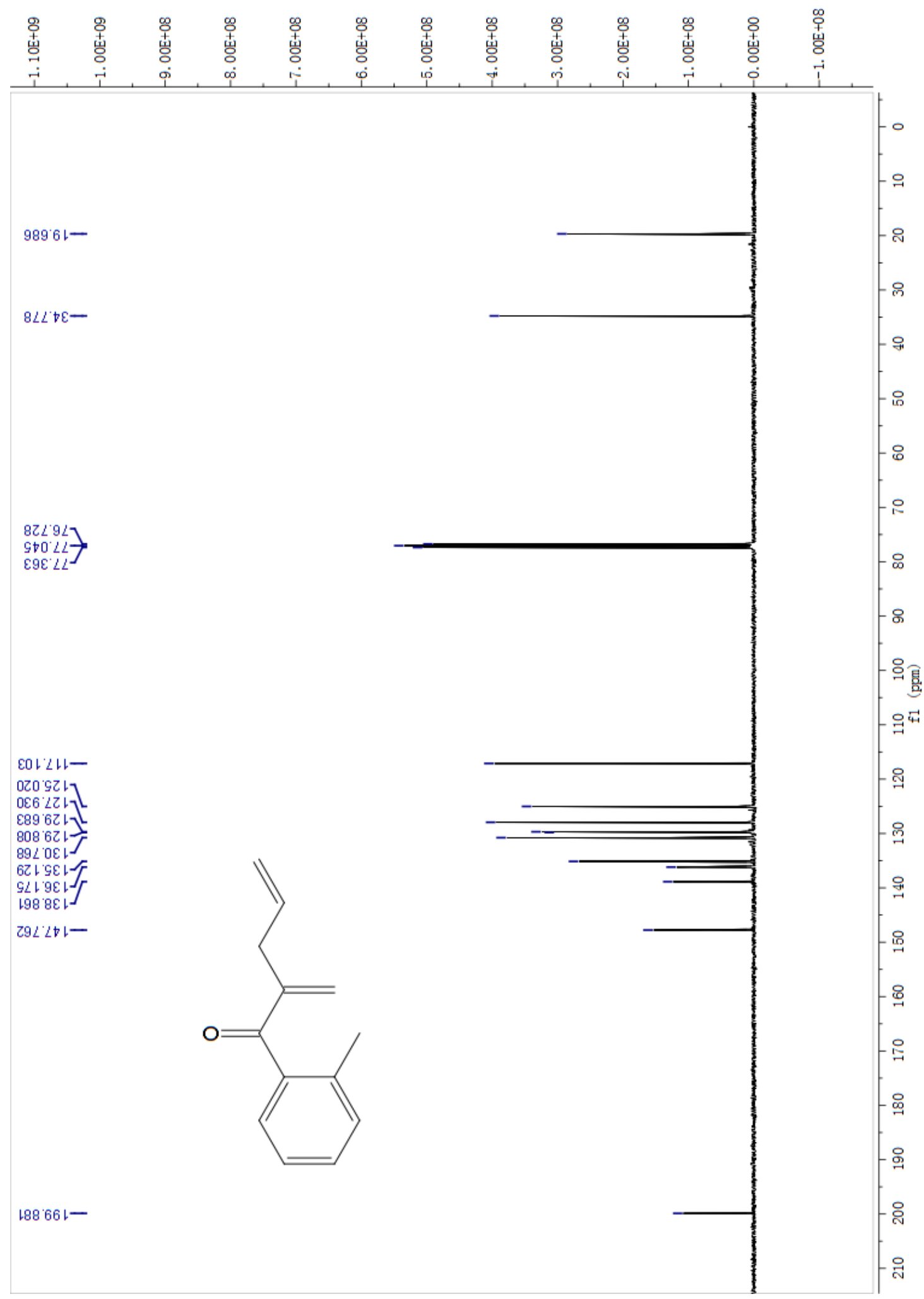


HR-MS Spectrum of 2-Methylene-1-(2-tolyl)pent-4-en-1-one 3ba

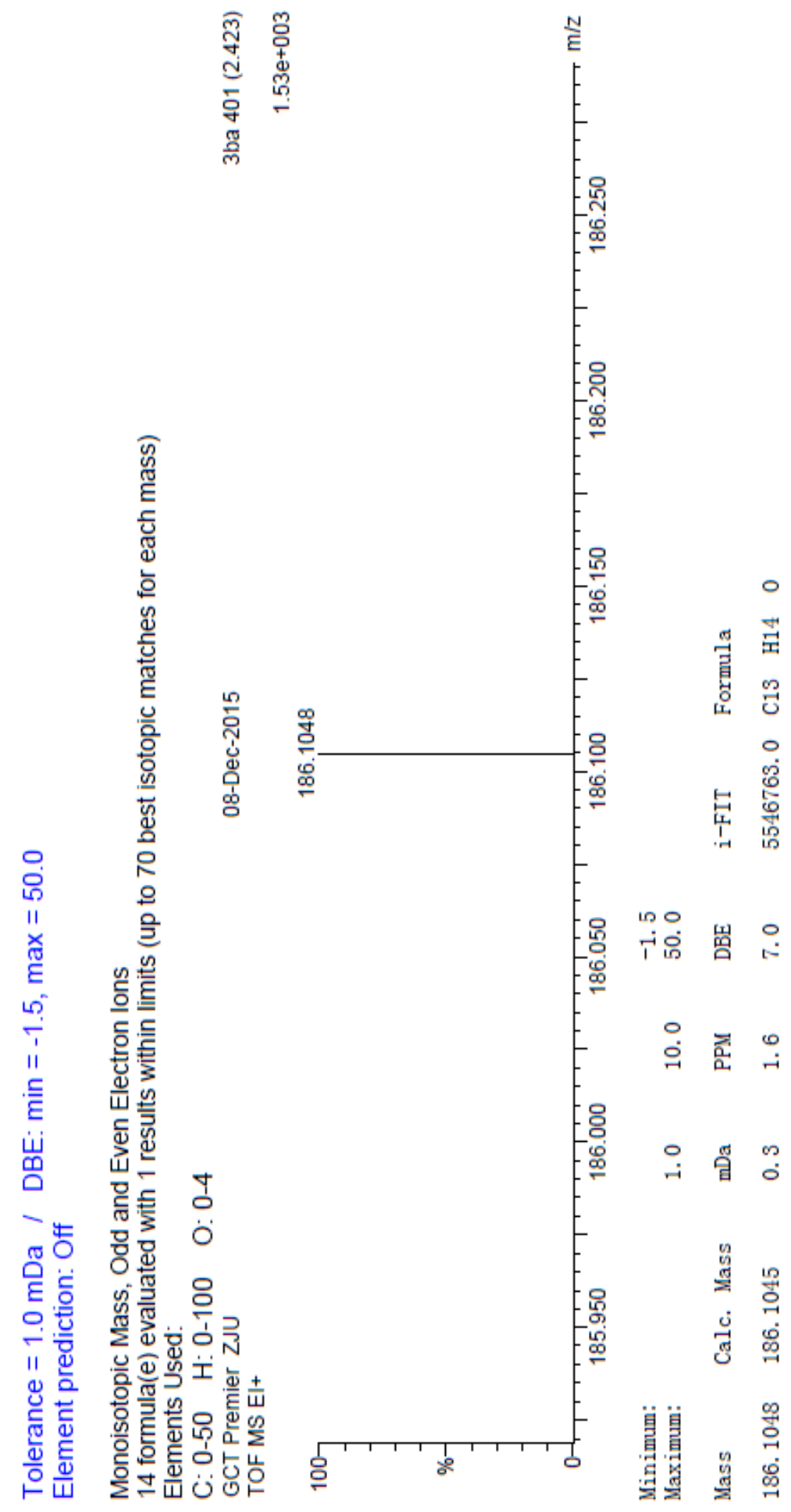


${ }^{1}$ H NMR Spectrum of 2-Methylene-1-(3-tolyl)pent-4-en-1-one 3ca

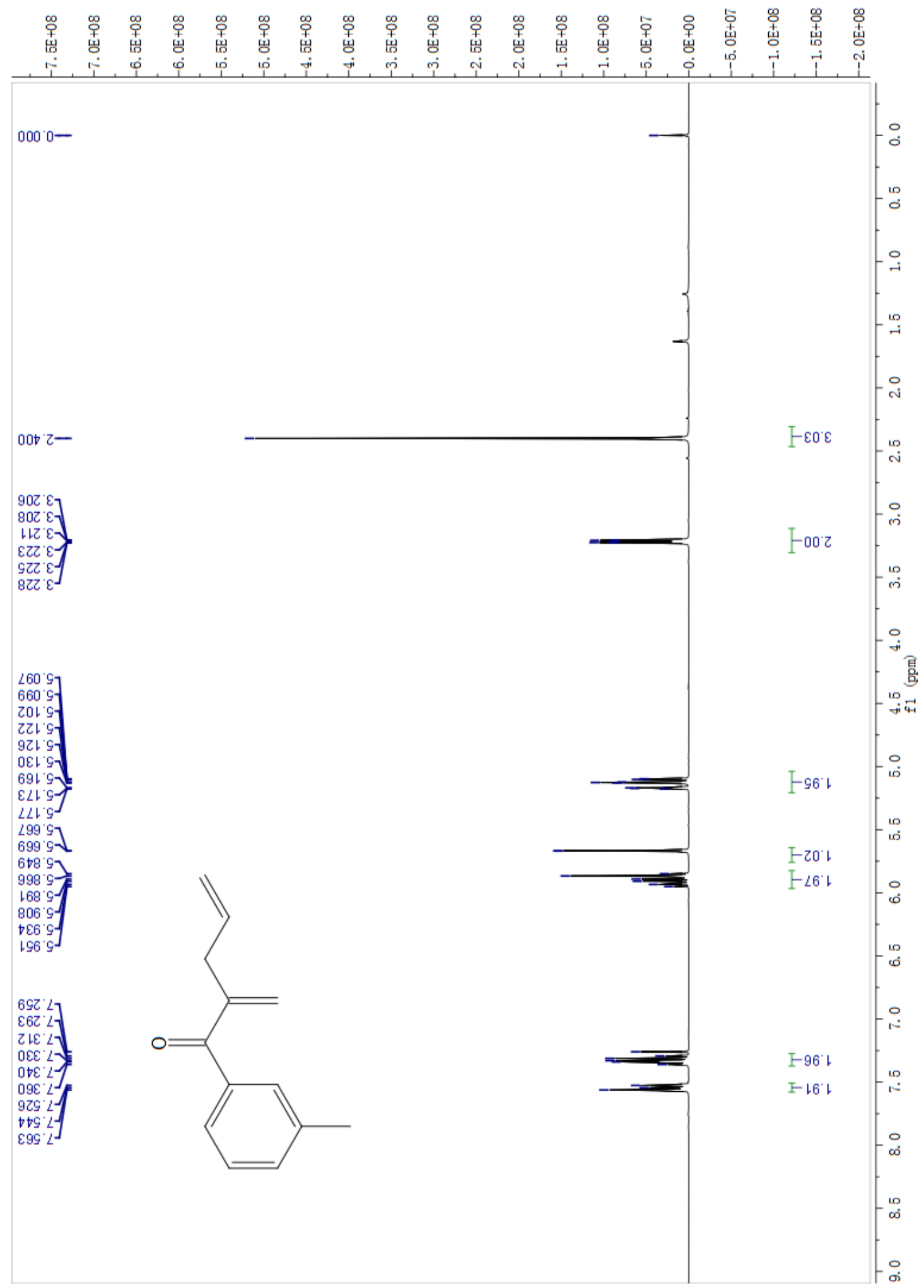


${ }^{13}$ C NMR Spectrum of 2-Methylene-1-(3-tolyl)pent-4-en-1-one 3ca

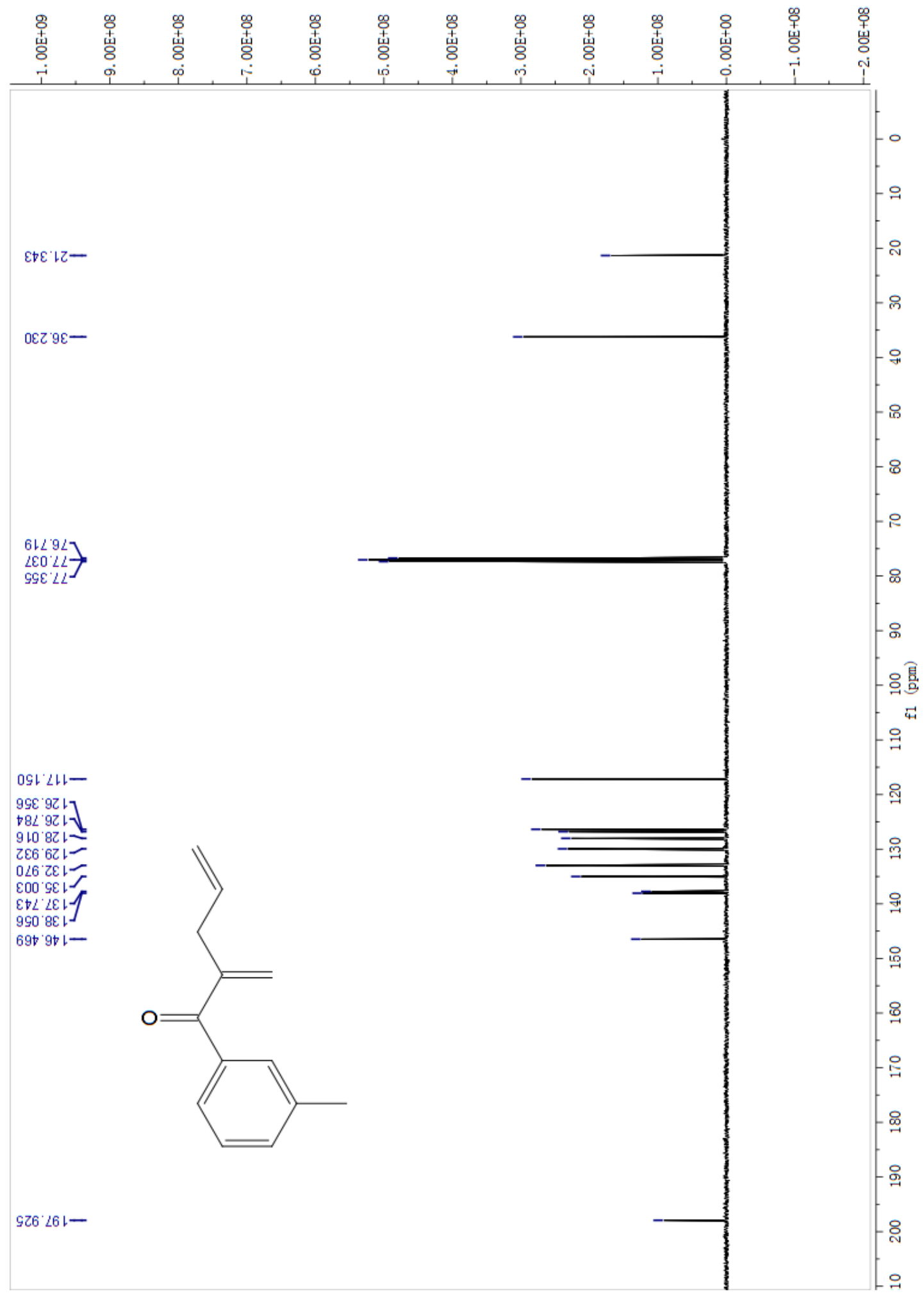


HR-MS Spectrum of 2-Methylene-1-(3-tolyl)pent-4-en-1-one 3ca

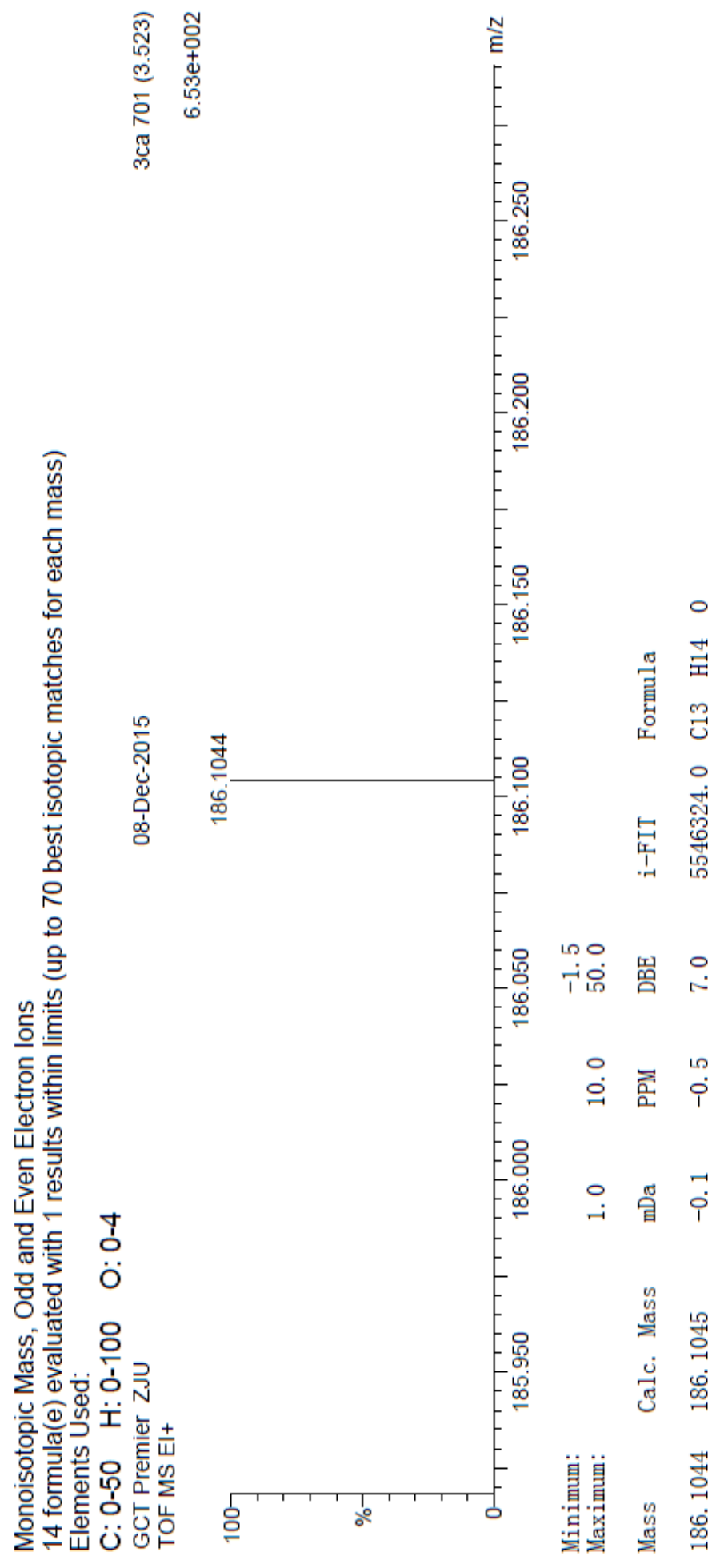


${ }^{1}$ H NMR Spectrum of 1-(3-Methoxyphenyl)-2-methylenepent-4-en-1-one 3da

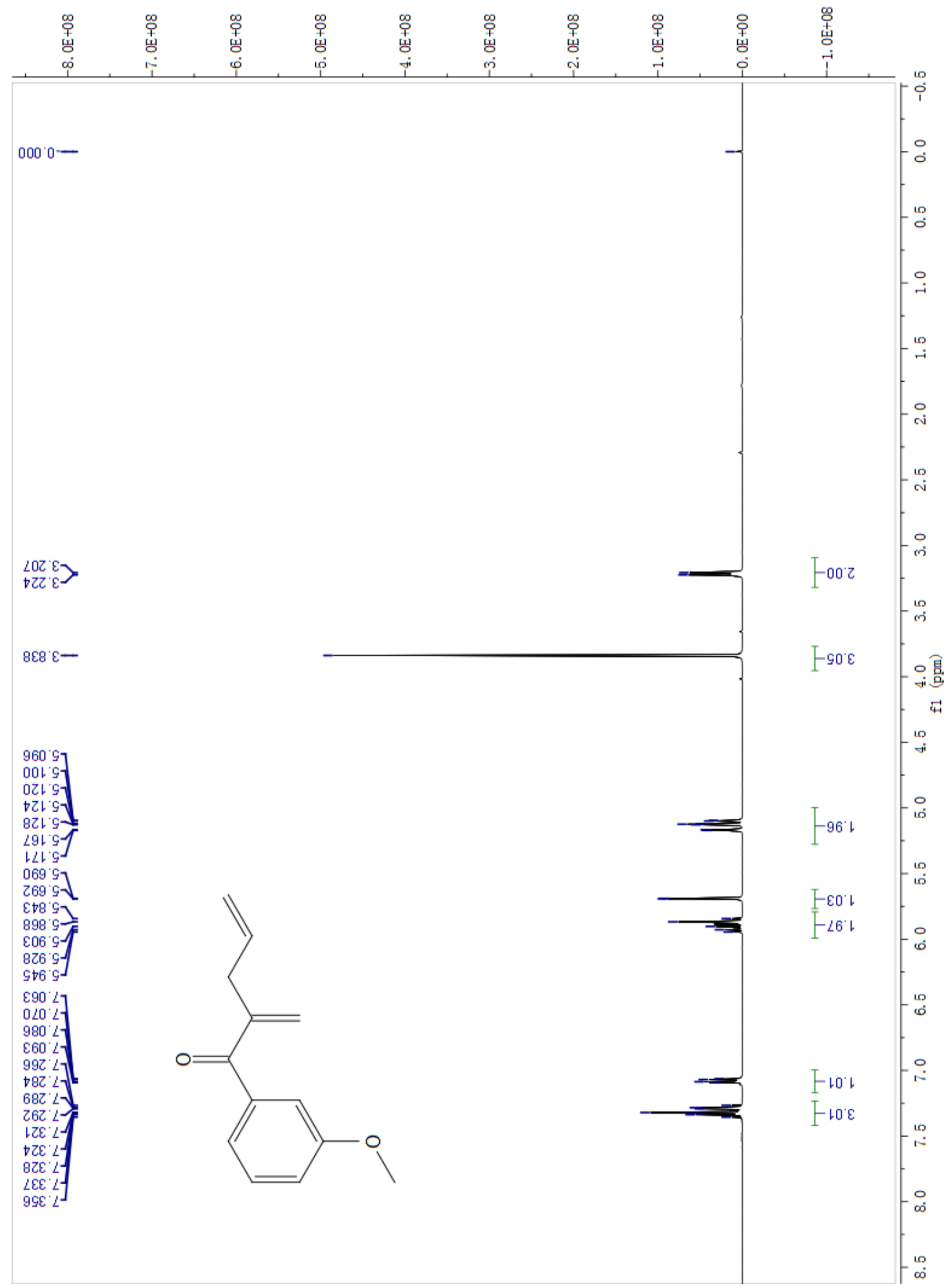


${ }^{13}$ C NMR Spectrum of 1-(3-Methoxyphenyl)-2-methylenepent-4-en-1-one 3da

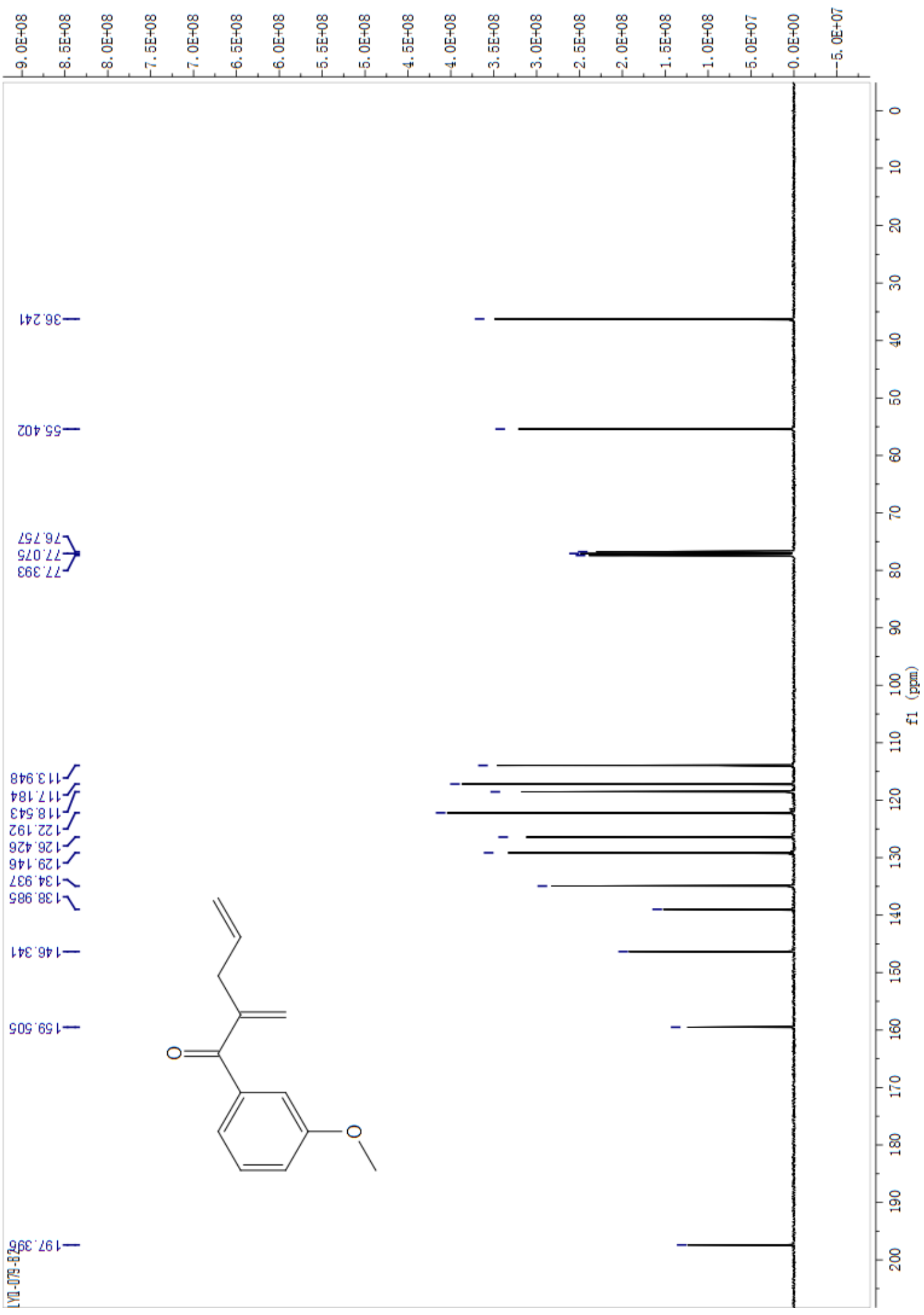


HR-MS Spectrum of 1-(3-Methoxyphenyl)-2-methylenepent-4-en-1-one 3da

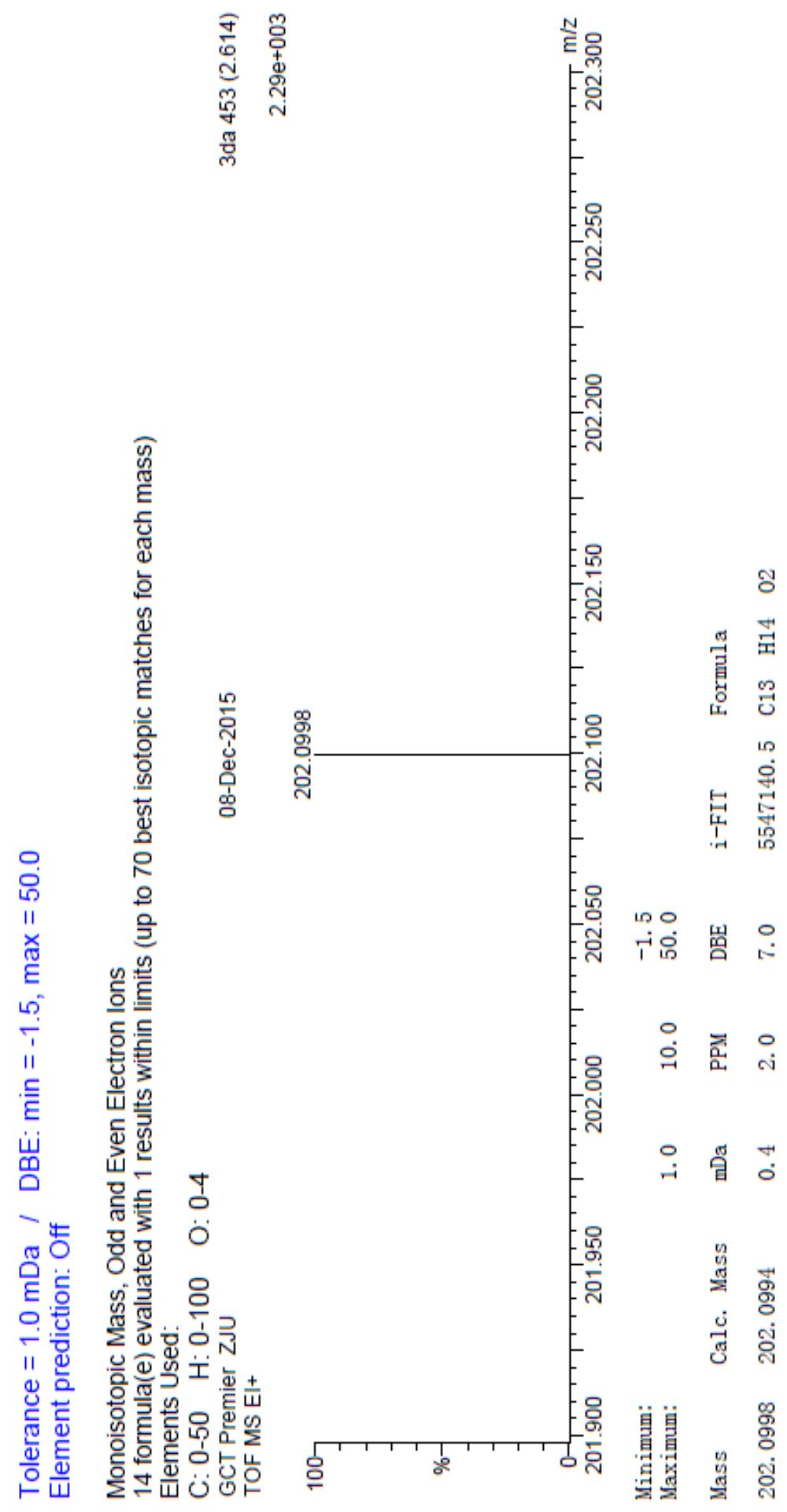


${ }^{1}$ H NMR Spectrum of 1-(4-Methoxyphenyl)-2-methylenepent-4-en-1-one 3ea

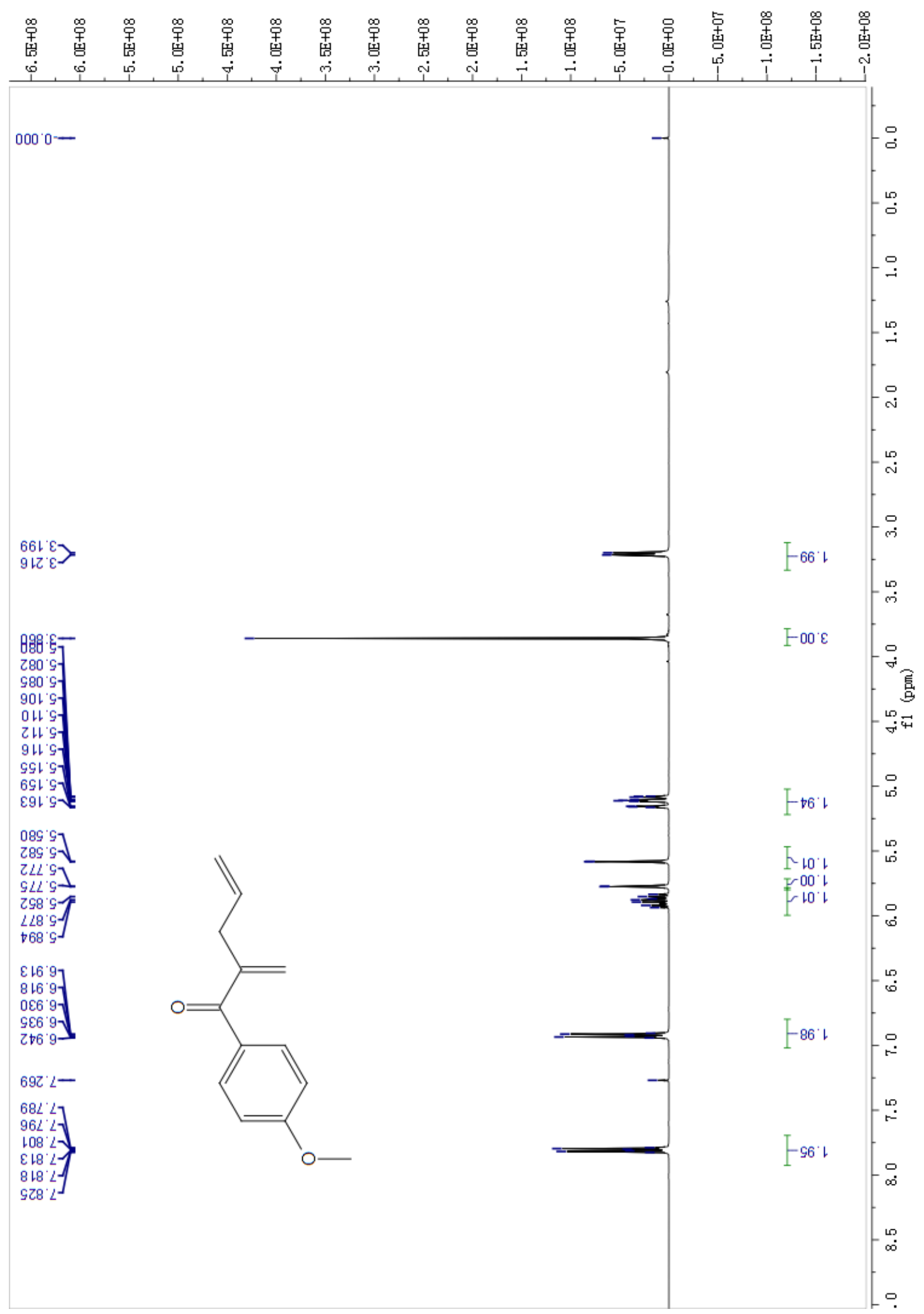


${ }^{13}$ C NMR Spectrum of 1-(4-Methoxyphenyl)-2-methylenepent-4-en-1-one 3ea

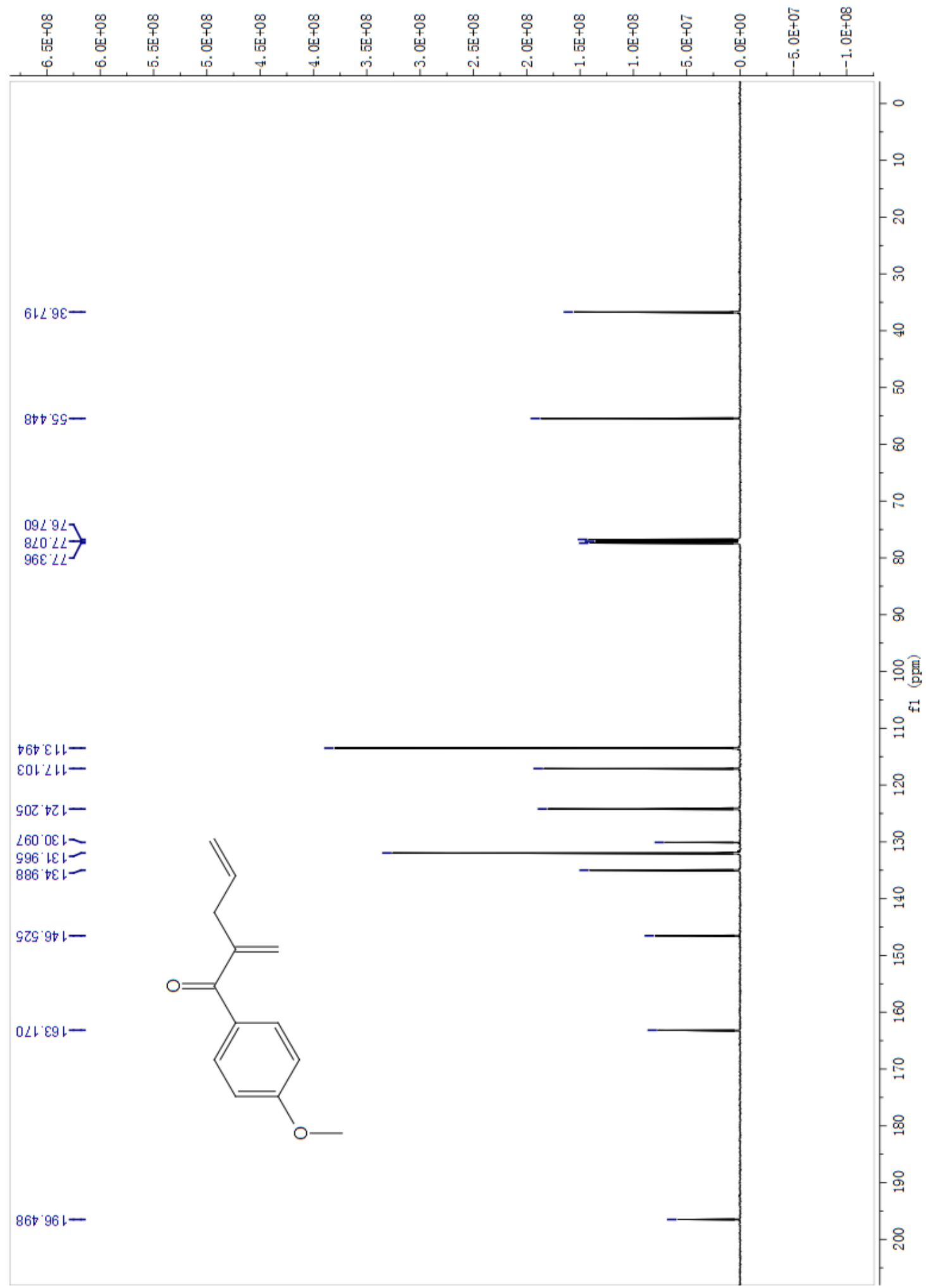


HR-MS Spectrum of 1-(4-Methoxyphenyl)-2-methylenepent-4-en-1-one 3ea

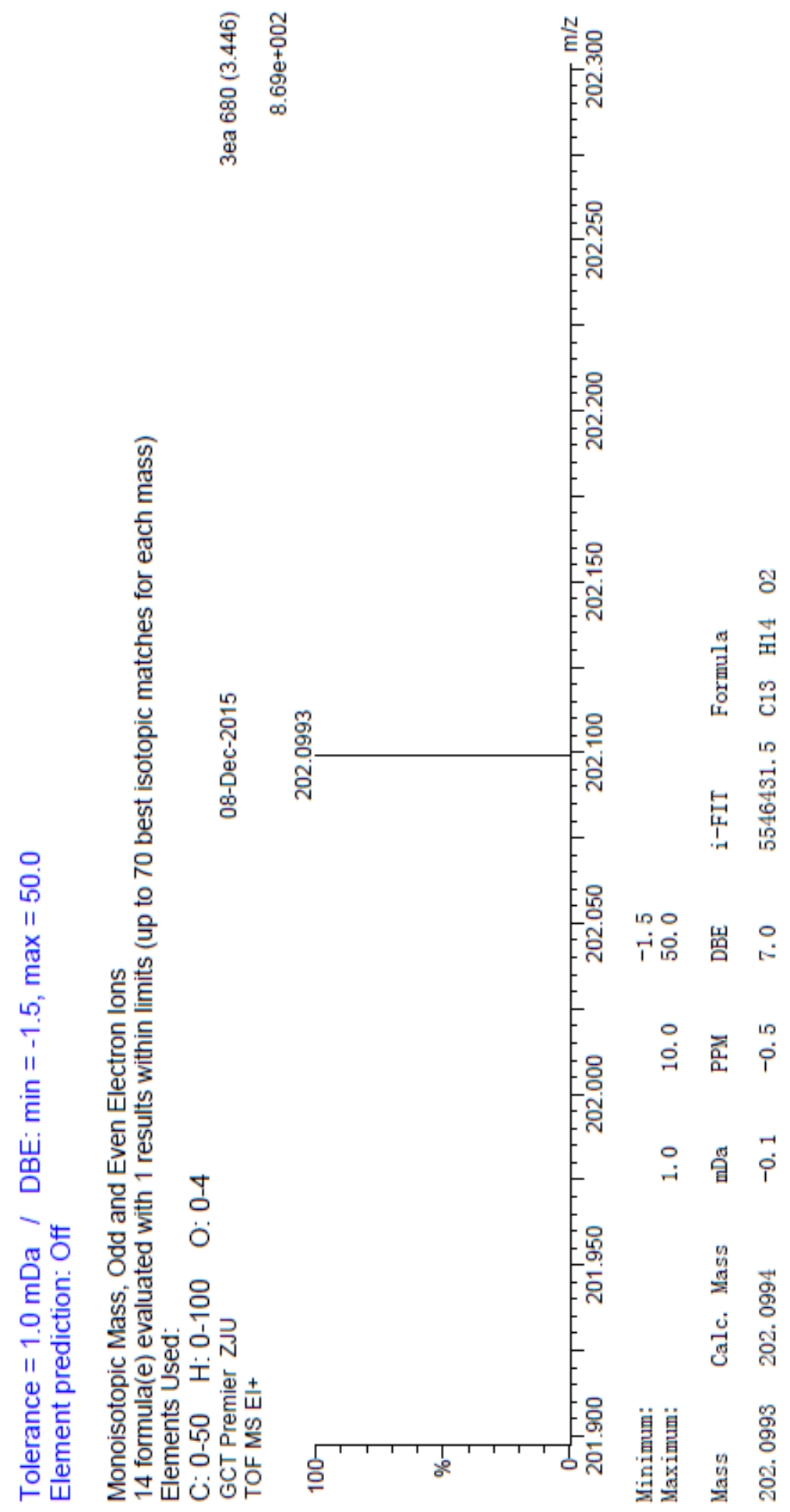


${ }^{1} \mathrm{H}$ NMR Spectrum of 2-Methylene-1-phenylpent-4-en-1-one 3fa

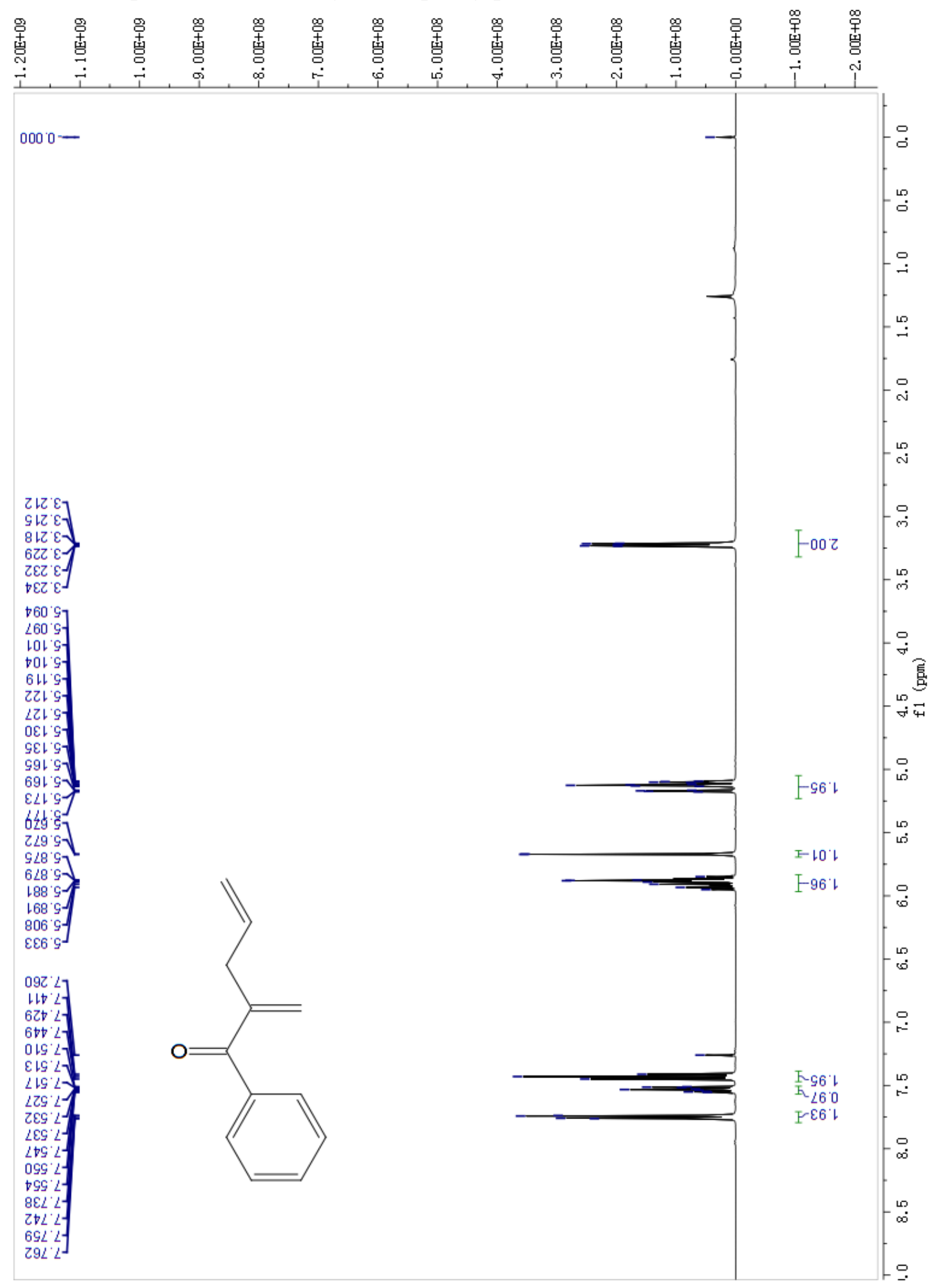


${ }^{13} \mathrm{C}$ NMR Spectrum of 2-Methylene-1-phenylpent-4-en-1-one 3fa

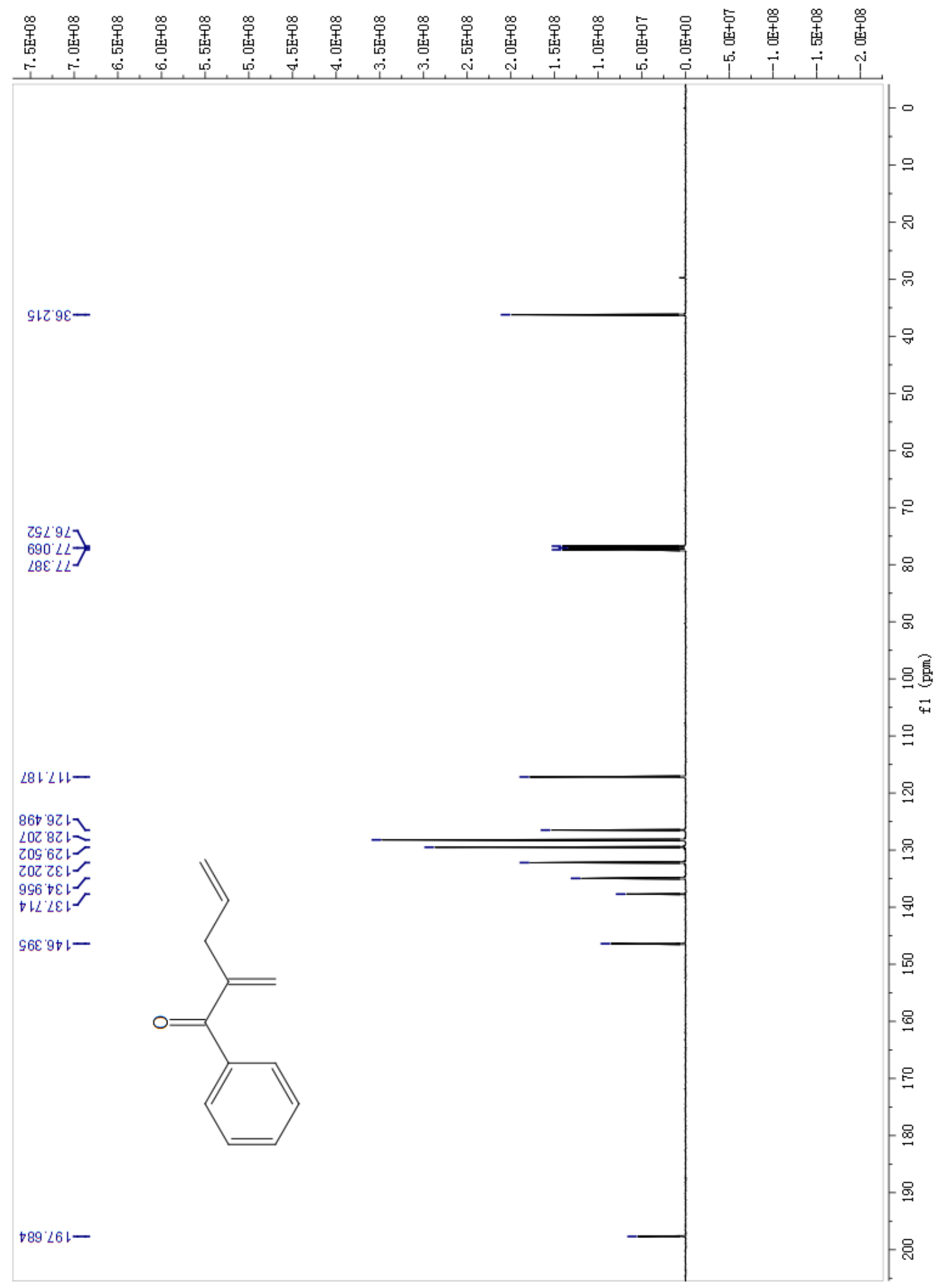


MS(EI) Spectrum of 2-Methylene-1-phenylpent-4-en-1-one 3fa

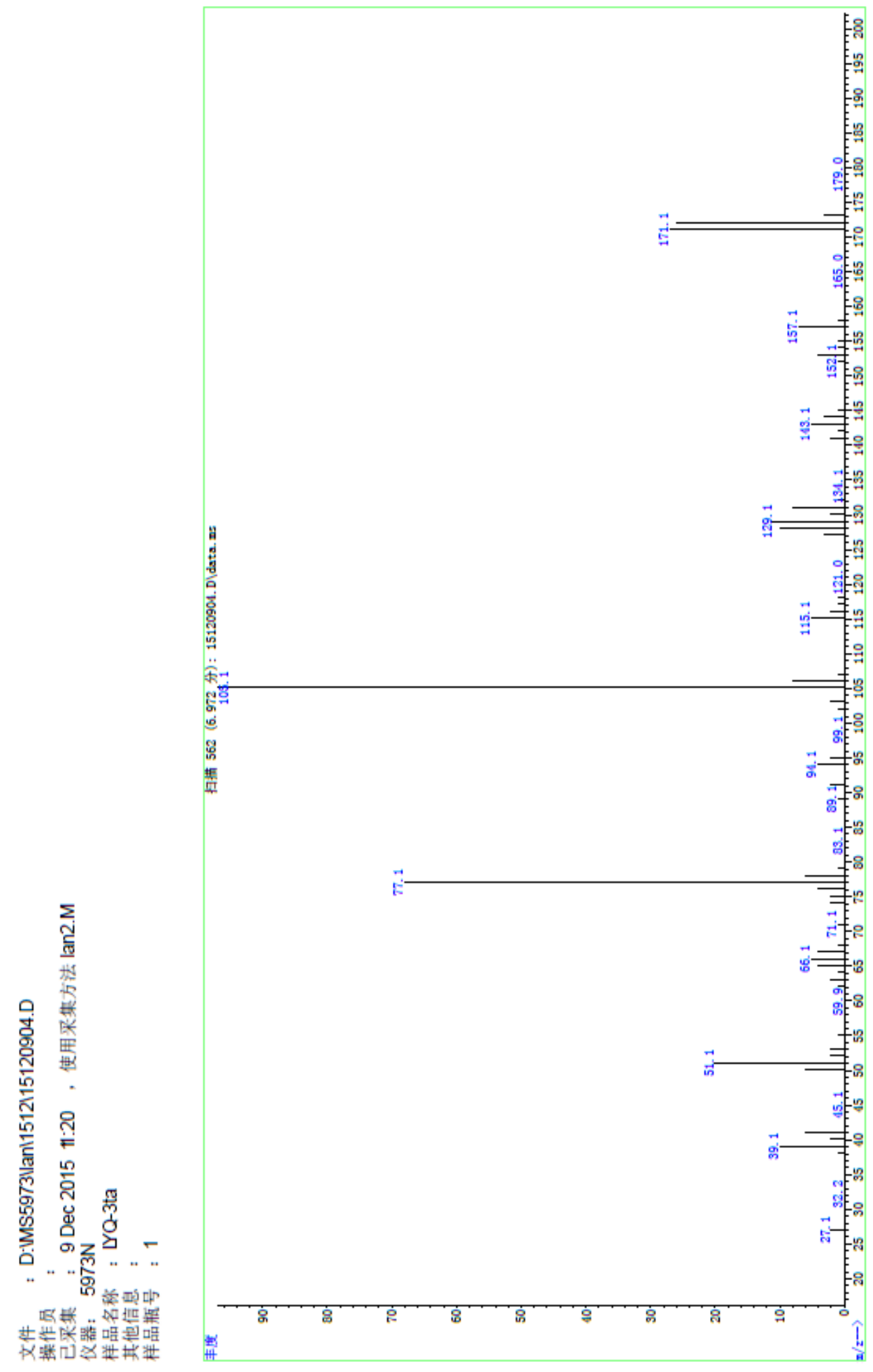


${ }^{1}$ H NMR Spectrum of 1-(4-Fluorophenyl)-2-methylenepent-4-en-1-one 3ga

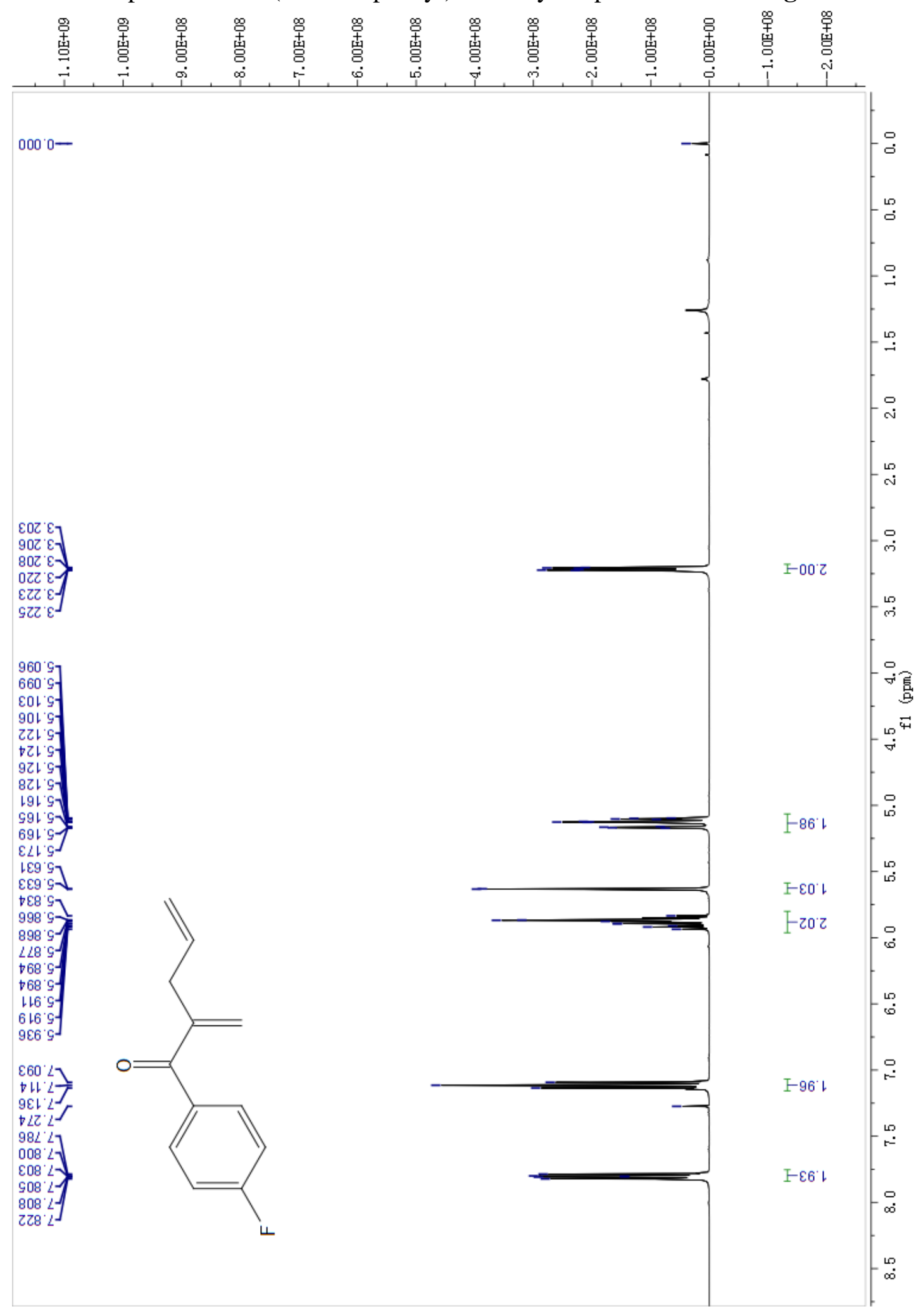


${ }^{13} \mathrm{C}$ NMR Spectrum of 1-(4-Fluorophenyl)-2-methylenepent-4-en-1-one 3ga

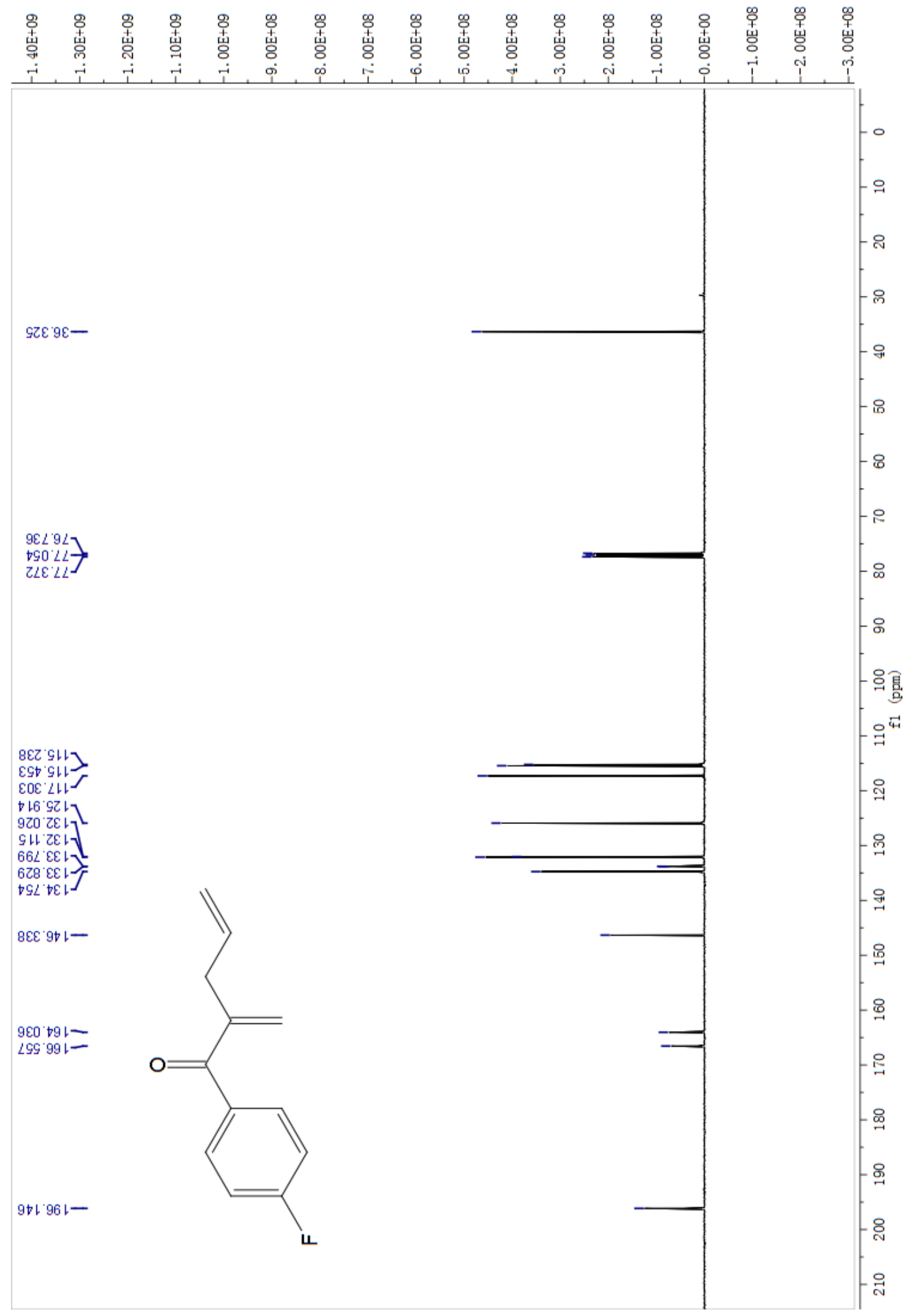


HR-MS Spectrum of 1-(4-Fluorophenyl)-2-methylenepent-4-en-1-one 3ga

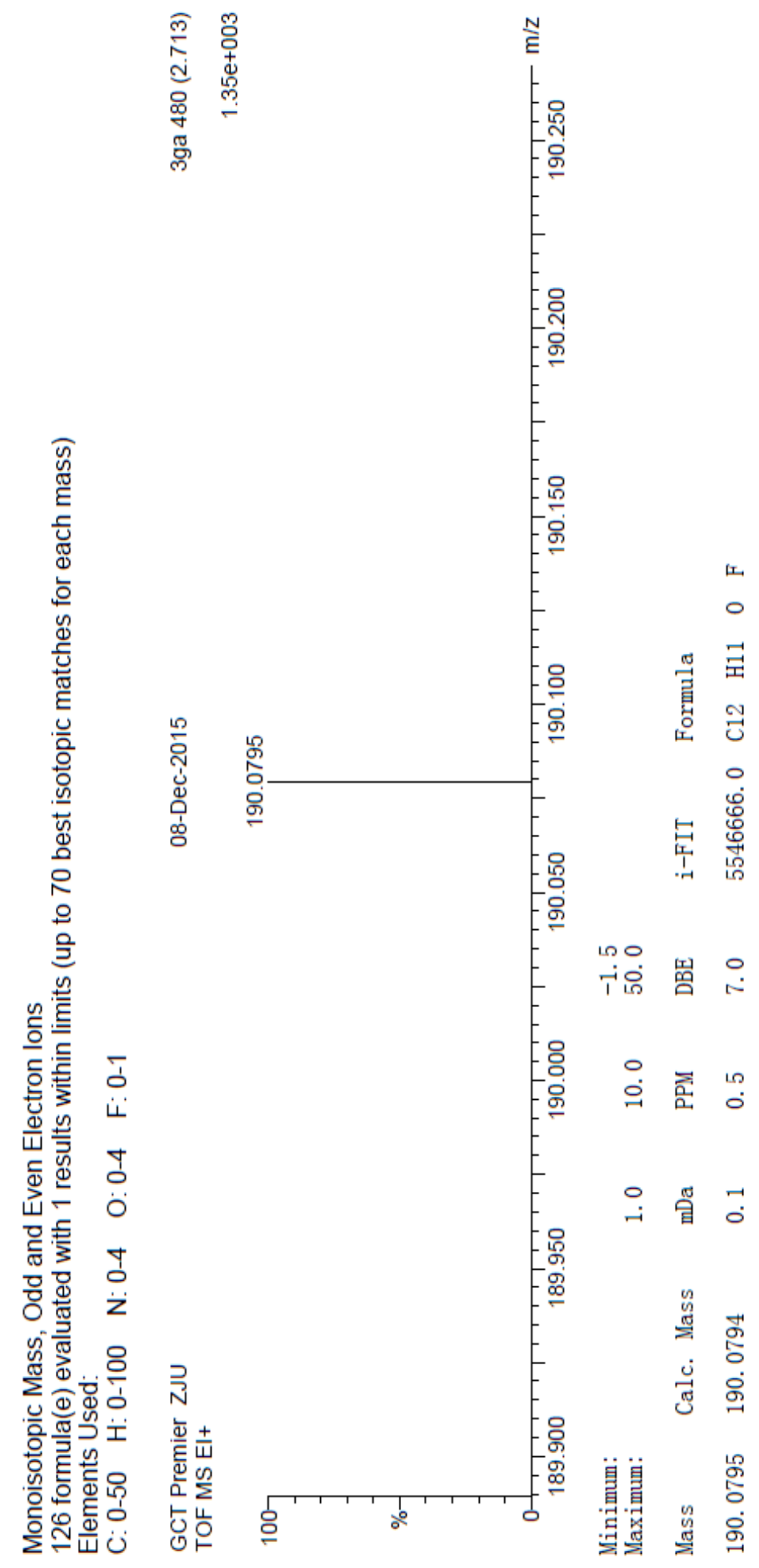


${ }^{1}$ H NMR Spectrum of 1-(4-Chlorophenyl)-2-methylenepent-4-en-1-one 3ha

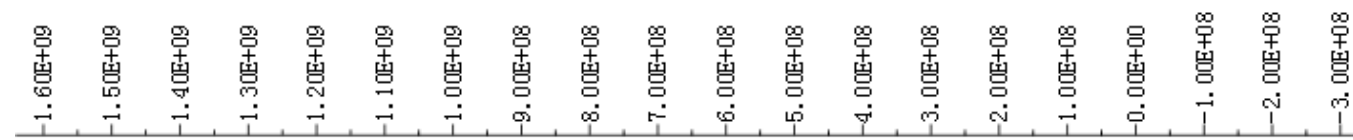

$0000-$
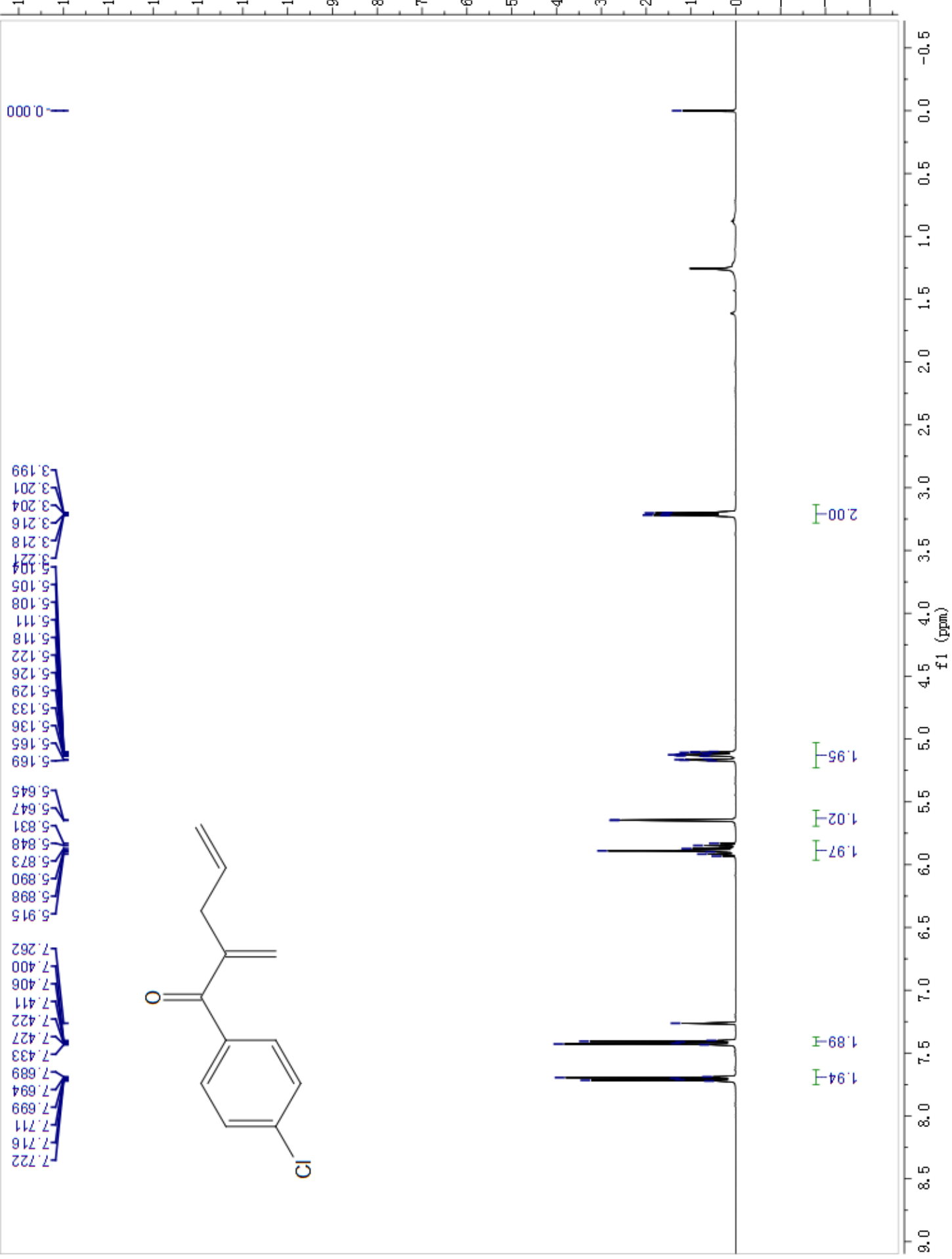
${ }^{13}$ C NMR Spectrum of 1-(4-Chlorophenyl)-2-methylenepent-4-en-1-one 3ha

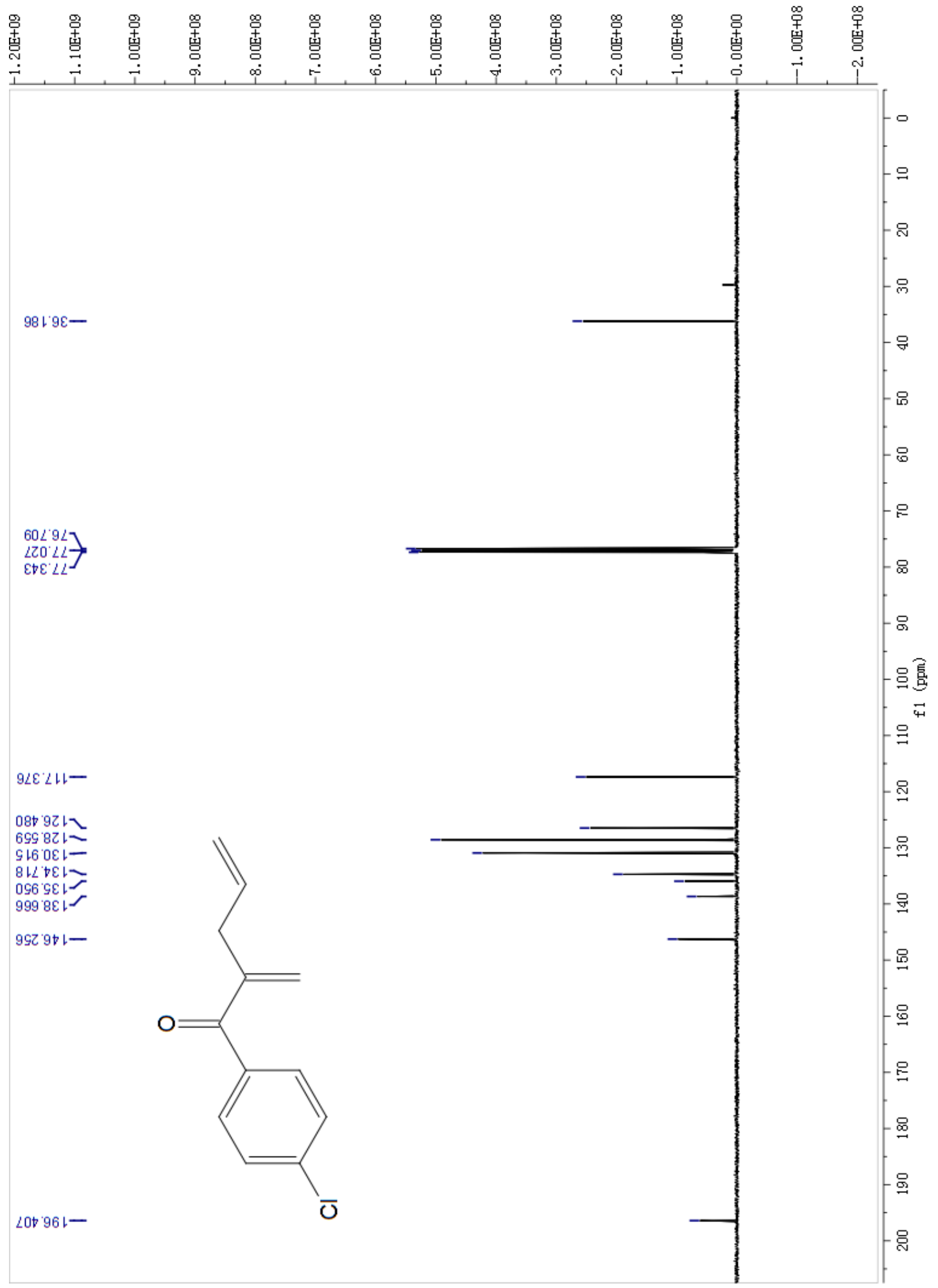


HR-MS Spectrum of 1-(4-Chlorophenyl)-2-methylenepent-4-en-1-one 3ha

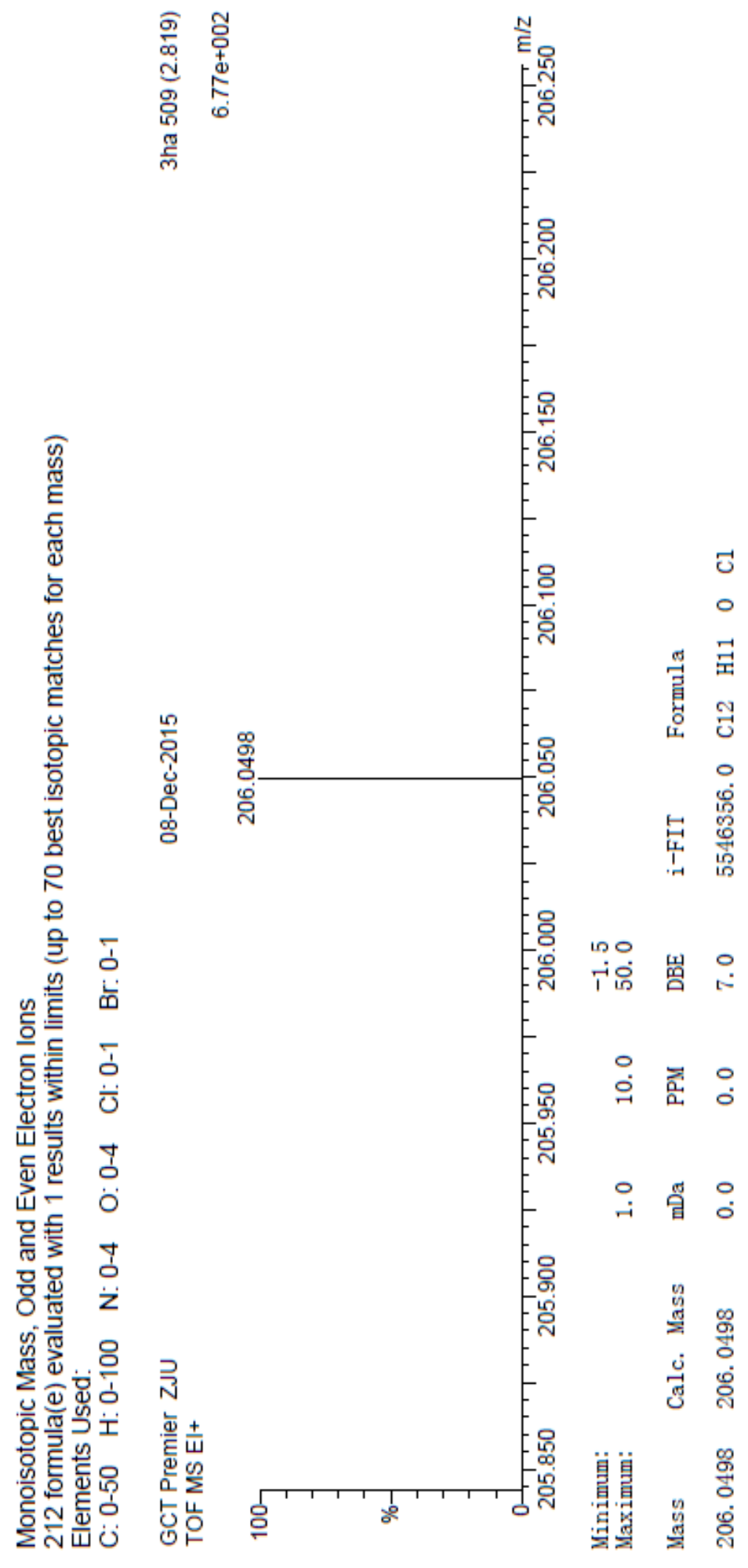


${ }^{1} \mathrm{H}$ NMR Spectrum of 1-(4-Bromophenyl)-2-methylenepent-4-en-1-one 3ia

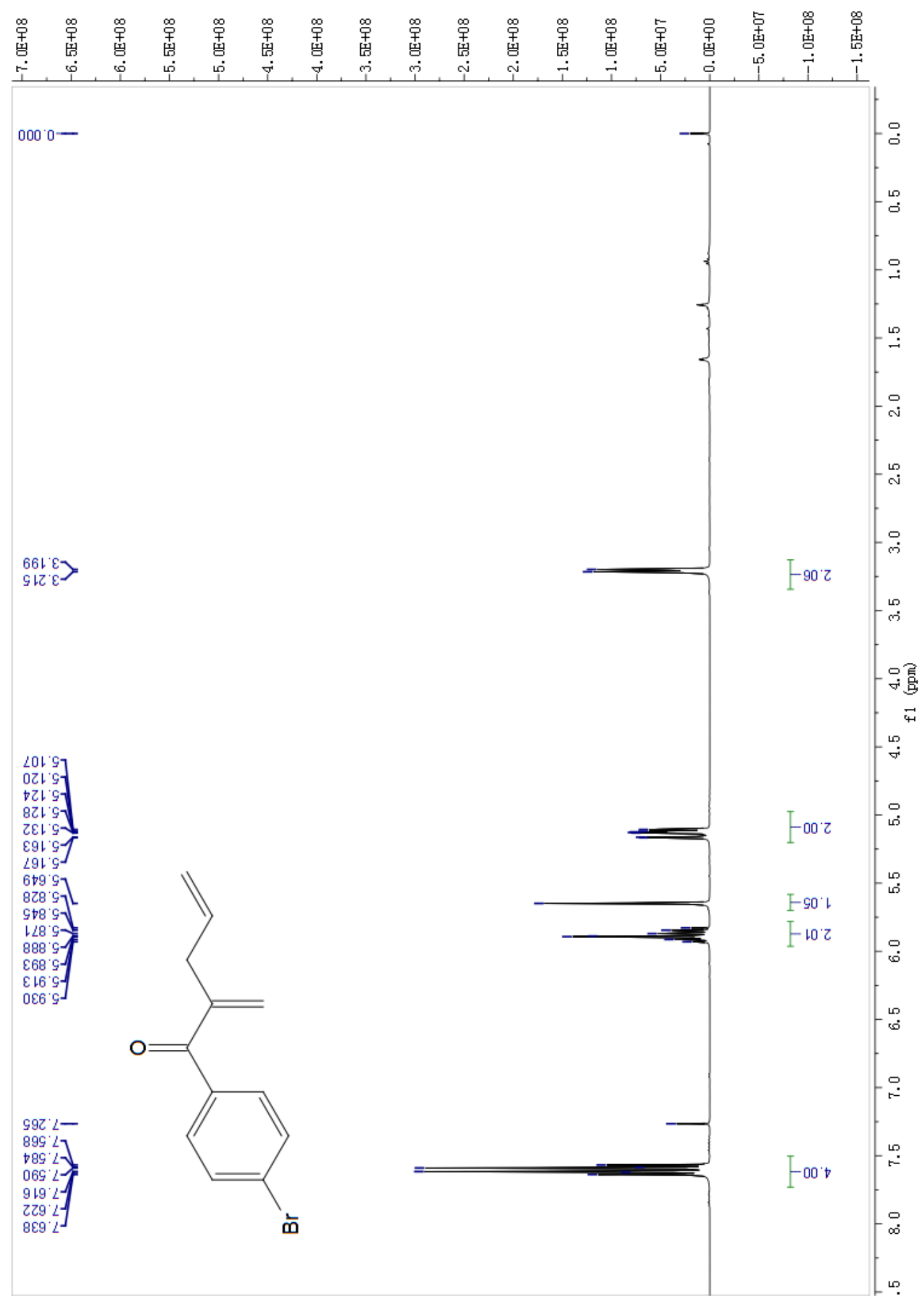


${ }^{13}$ C NMR Spectrum of 1-(4-Bromophenyl)-2-methylenepent-4-en-1-one 3ia

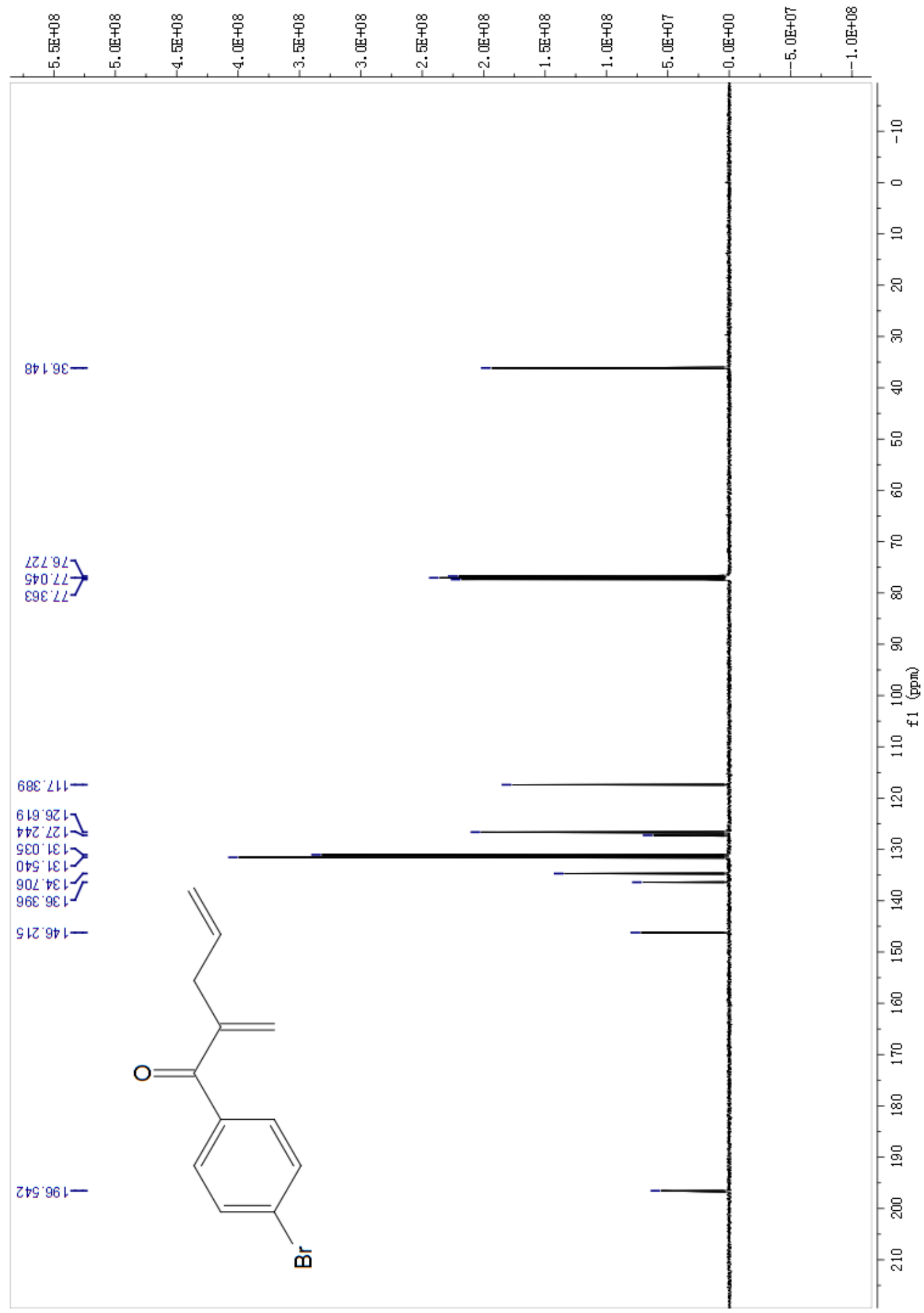


HR-MS Spectrum of 1-(4-Bromophenyl)-2-methylenepent-4-en-1-one 3ia

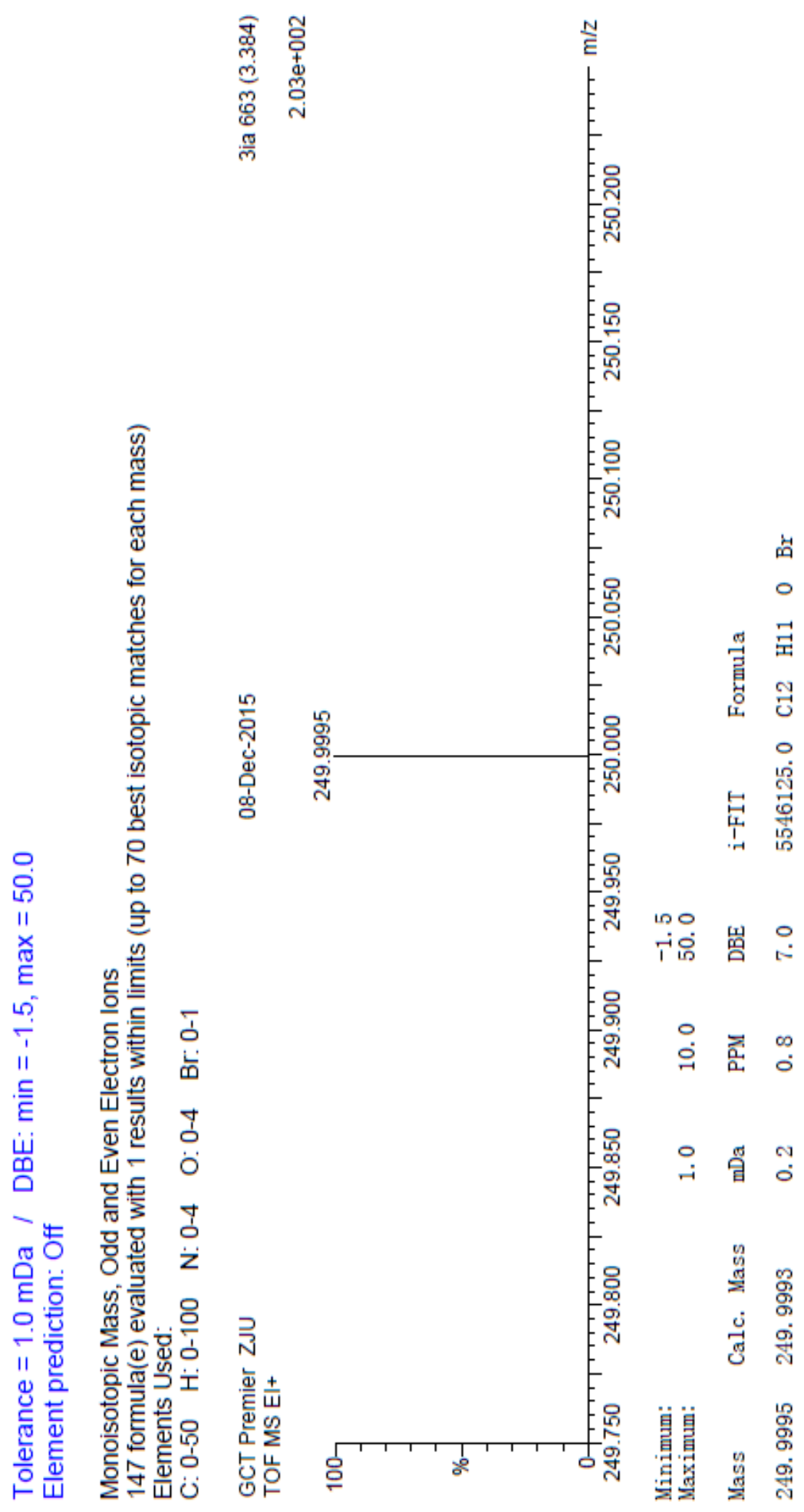


${ }^{1} \mathrm{H}$ NMR Spectrum of 2-Methylene-1-(3-nitrophenyl)pent-4-en-1-one 3ja

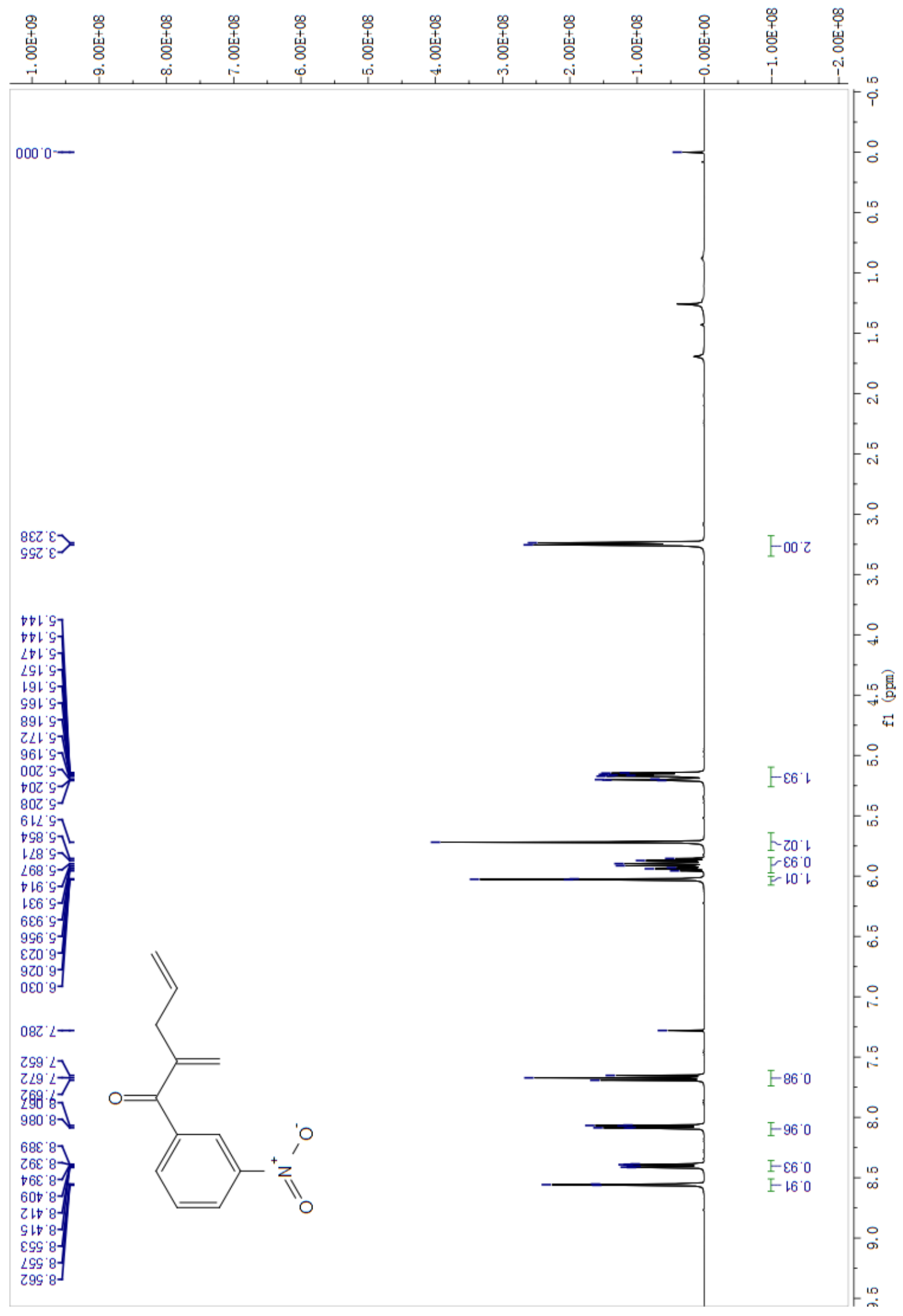


${ }^{13} \mathrm{C}$ NMR Spectrum of 2-Methylene-1-(3-nitrophenyl)pent-4-en-1-one 3ja

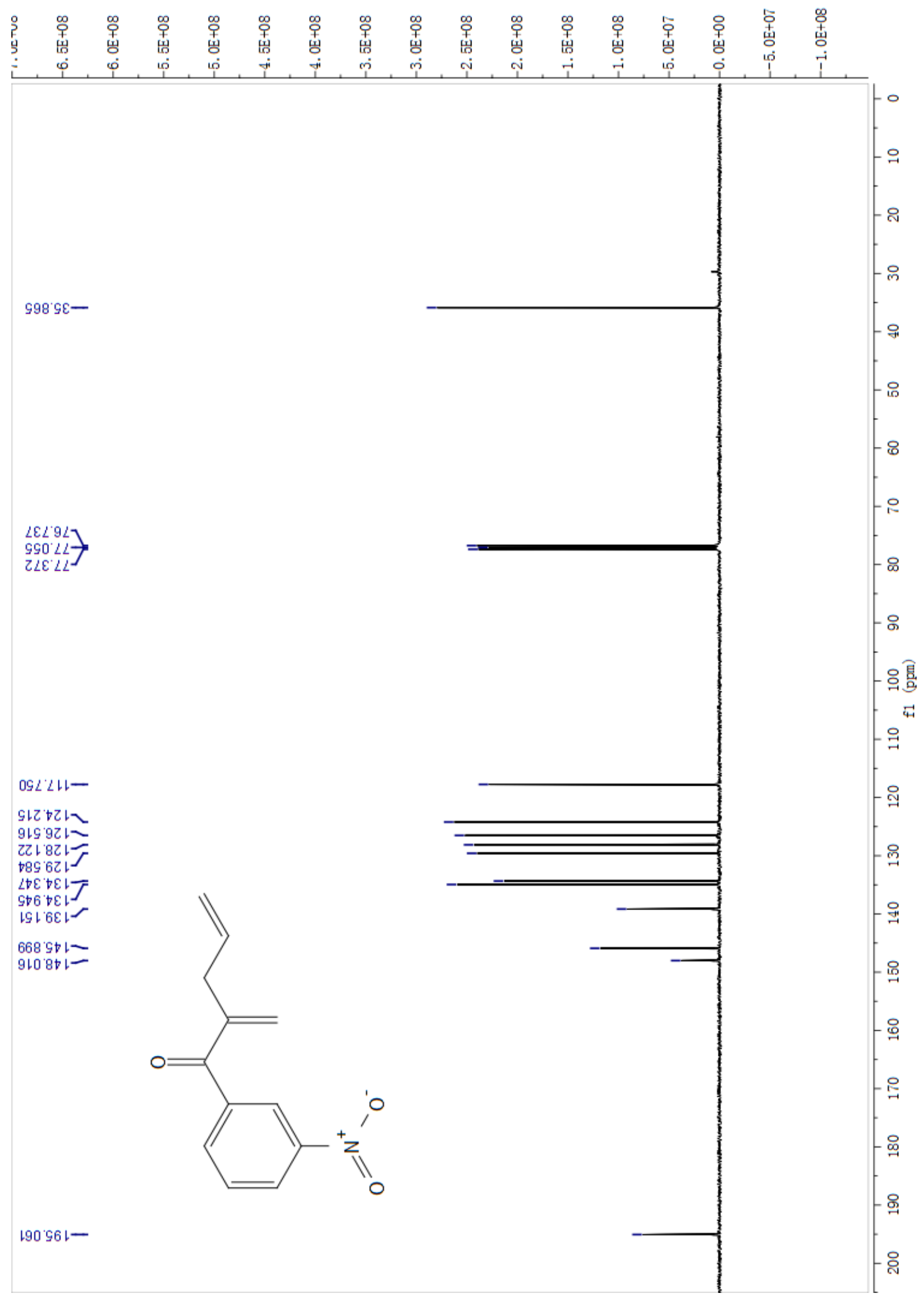


HR-MS Spectrum of 2-Methylene-1-(3-nitrophenyl)pent-4-en-1-one 3ja

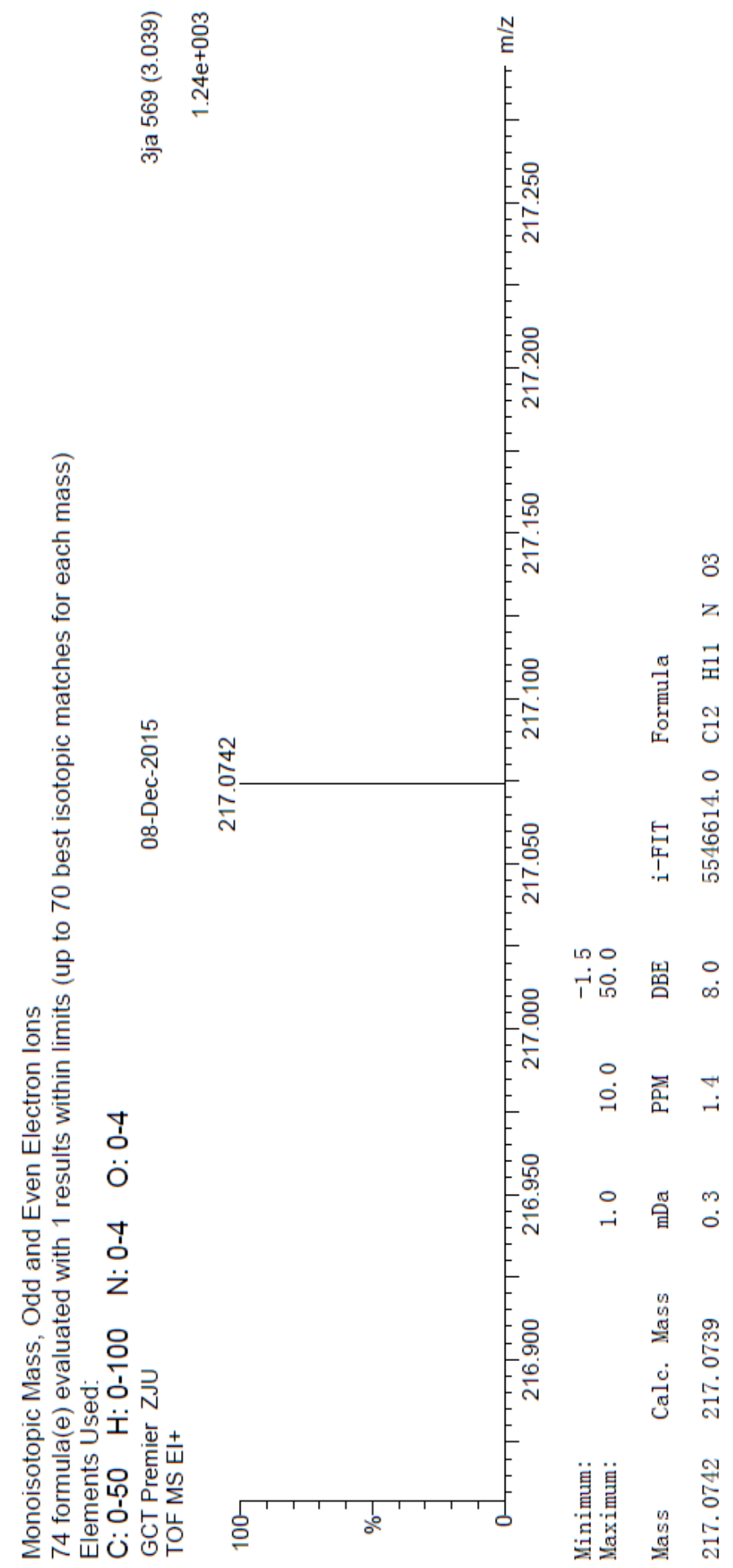


${ }^{1}$ H NMR Spectrum of 2-Methylene-1-(4-nitrophenyl)pent-4-en-1-one 3ka

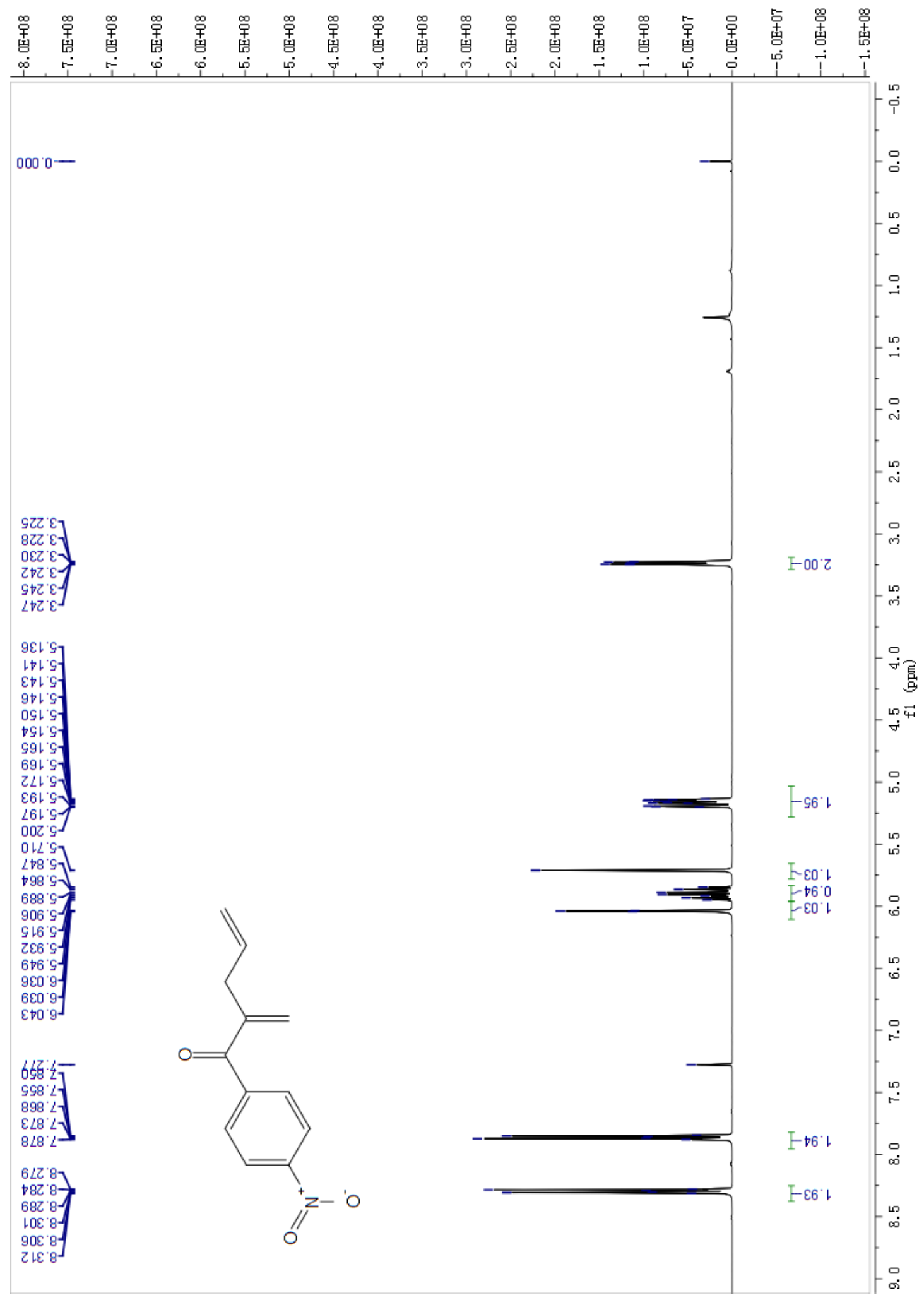


${ }^{13} \mathrm{C}$ NMR Spectrum of 2-Methylene-1-(4-nitrophenyl)pent-4-en-1-one 3ka

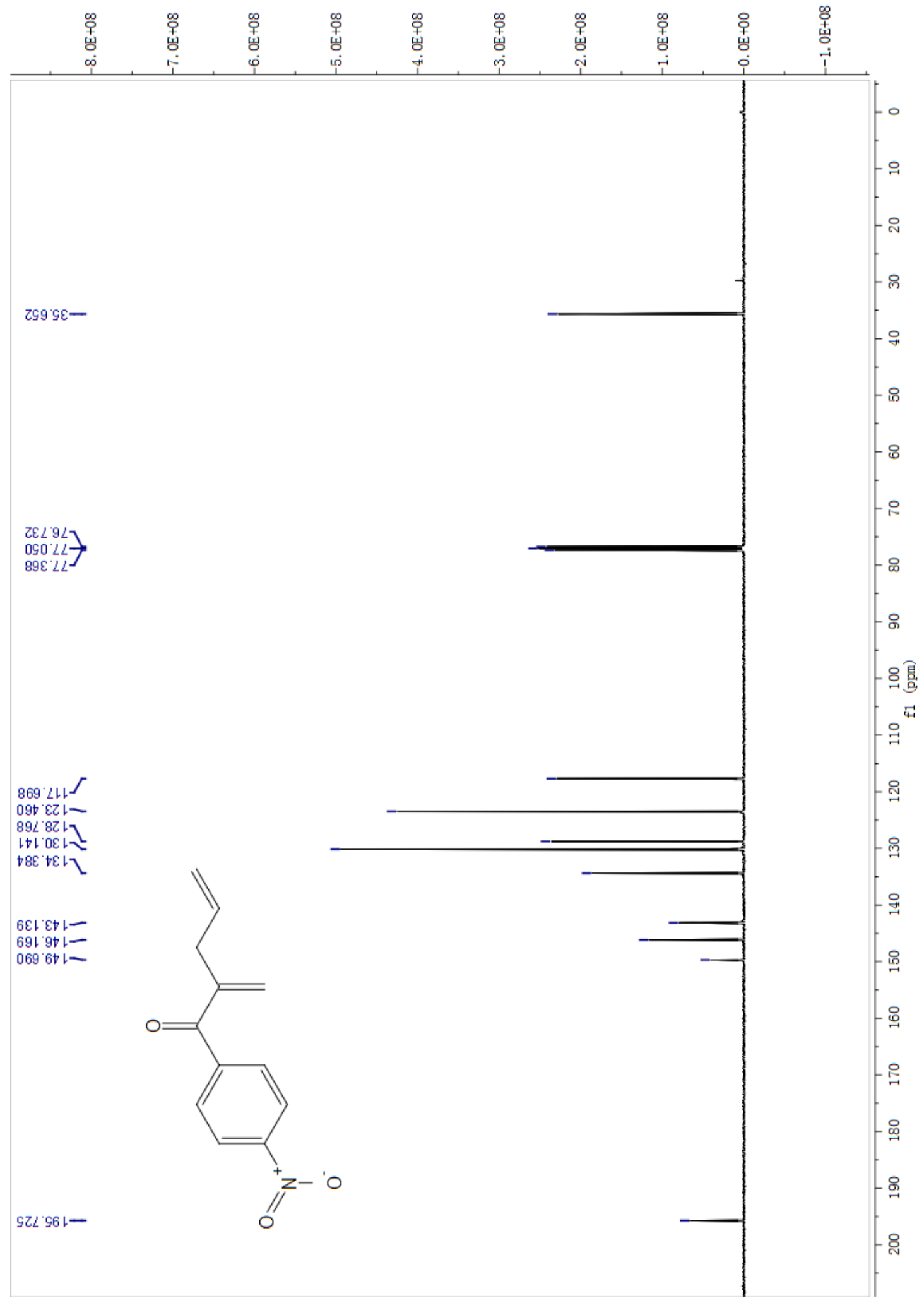


HR-MS Spectrum of 2-Methylene-1-(4-nitrophenyl)pent-4-en-1-one 3ka

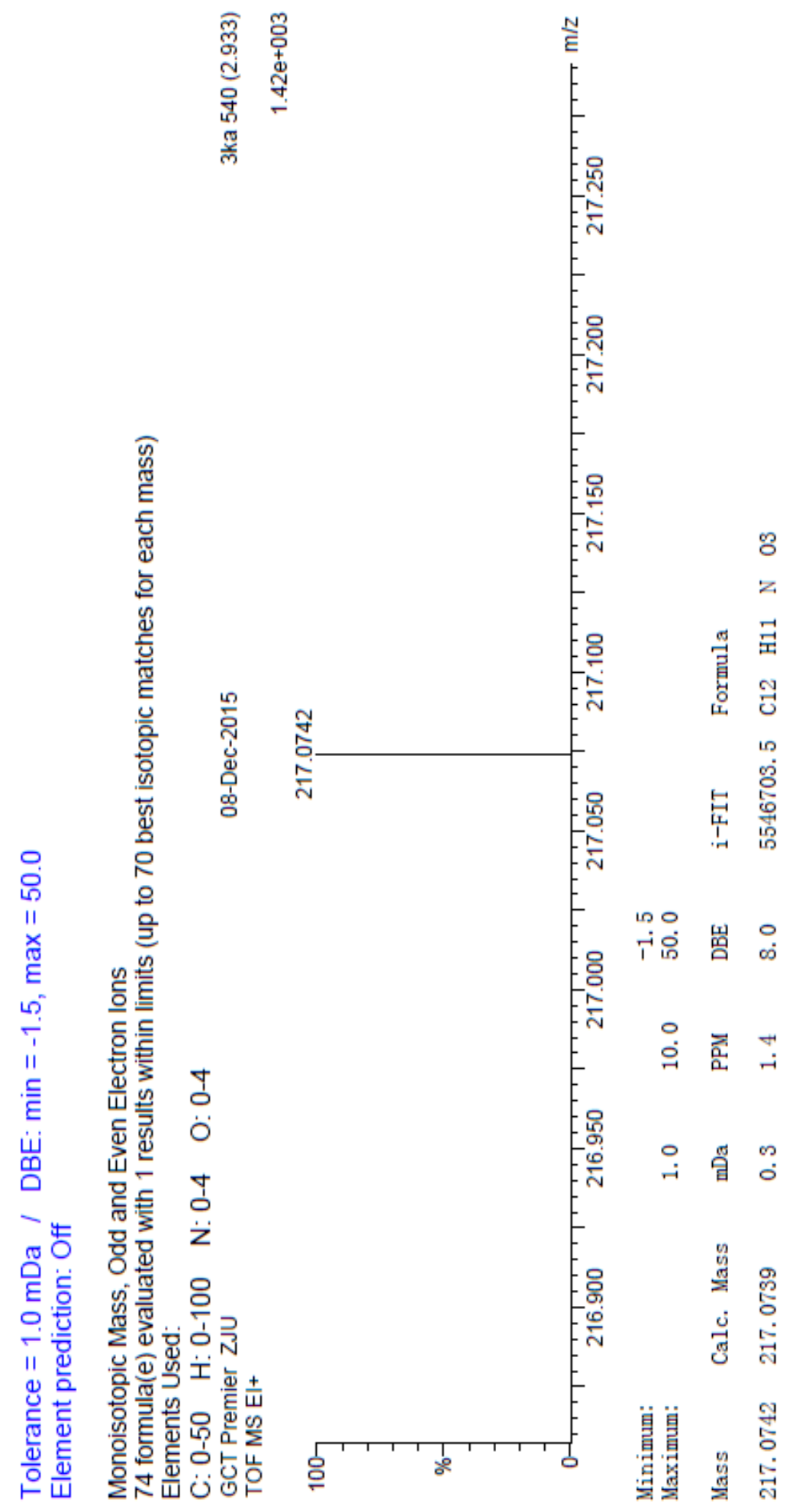


${ }^{1}$ H NMR Spectrum of 2-Methylene-1-(4-(trifluoromethyl)phenyl)pent-4-en-1-one 3la

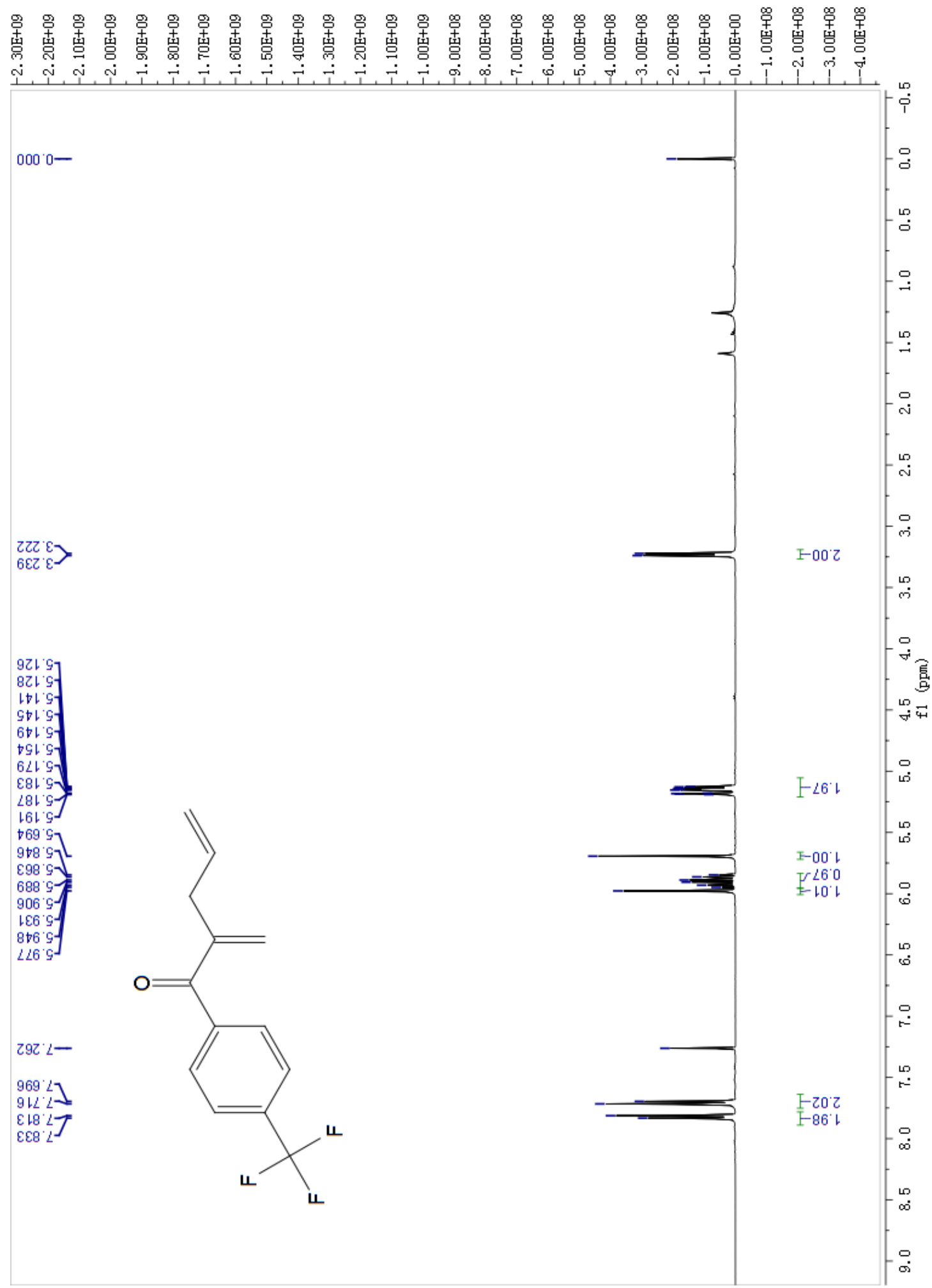


${ }^{13}$ C NMR Spectrum of 2-Methylene-1-(4-(trifluoromethyl)phenyl)pent-4-en-1-one 3la

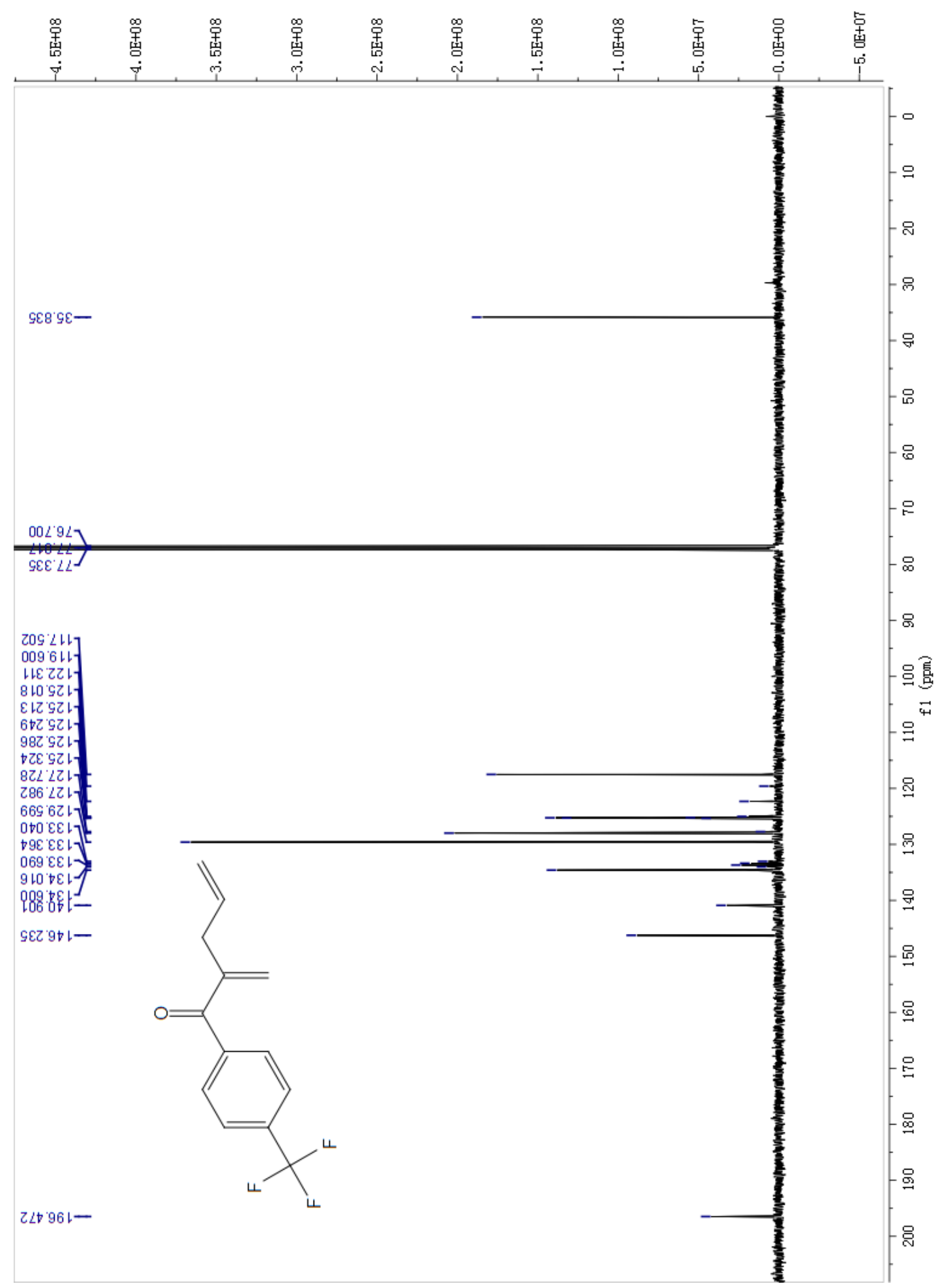


HR-MS Spectrum of 2-Methylene-1-(4-(trifluoromethyl)phenyl)pent-4-en-1-one 3la

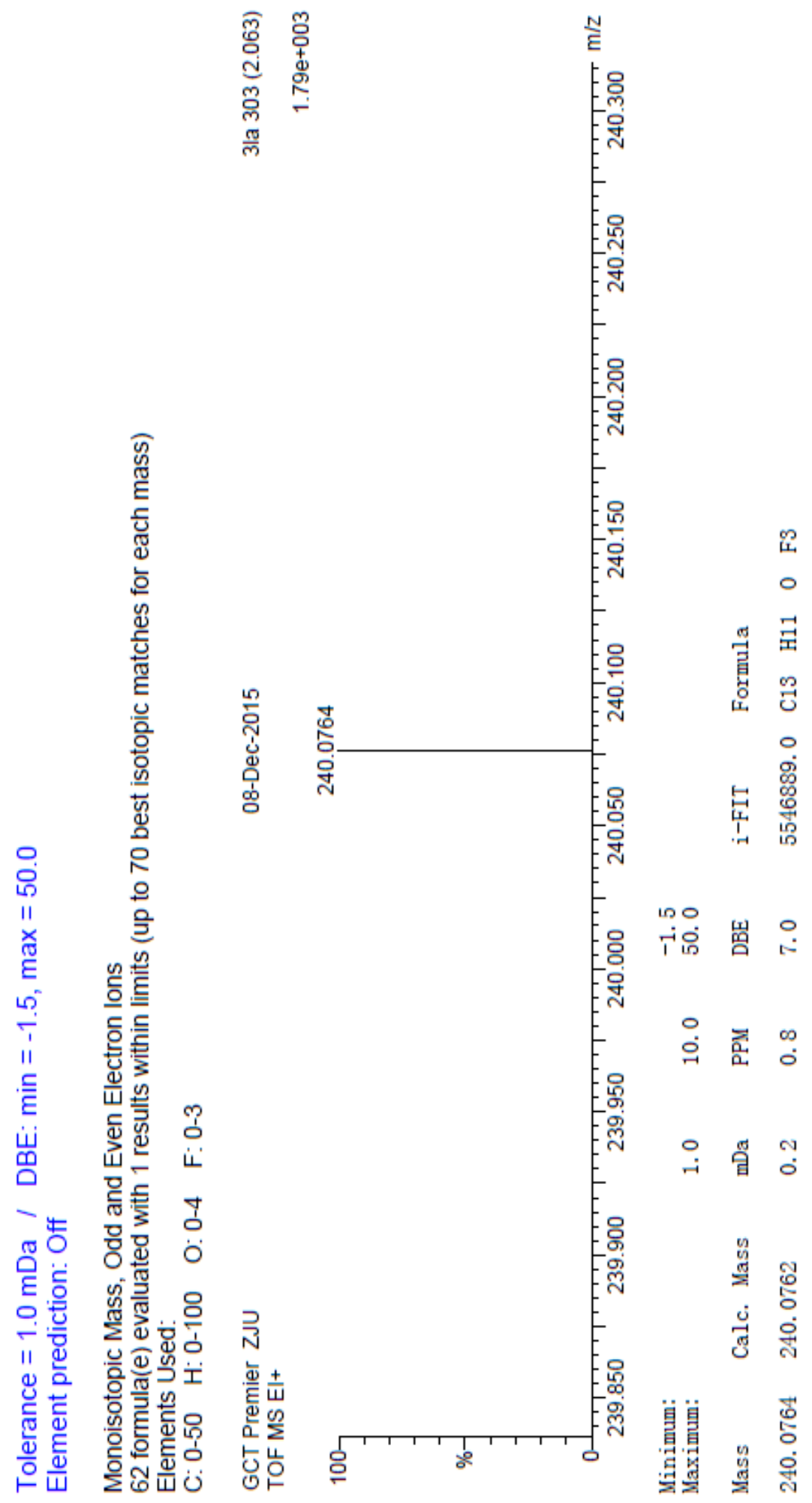


${ }^{1} \mathrm{H}$ NMR Spectrum of 2-Methylene-1-(naphthalen-2-yl)pent-4-en-1-one 3ma

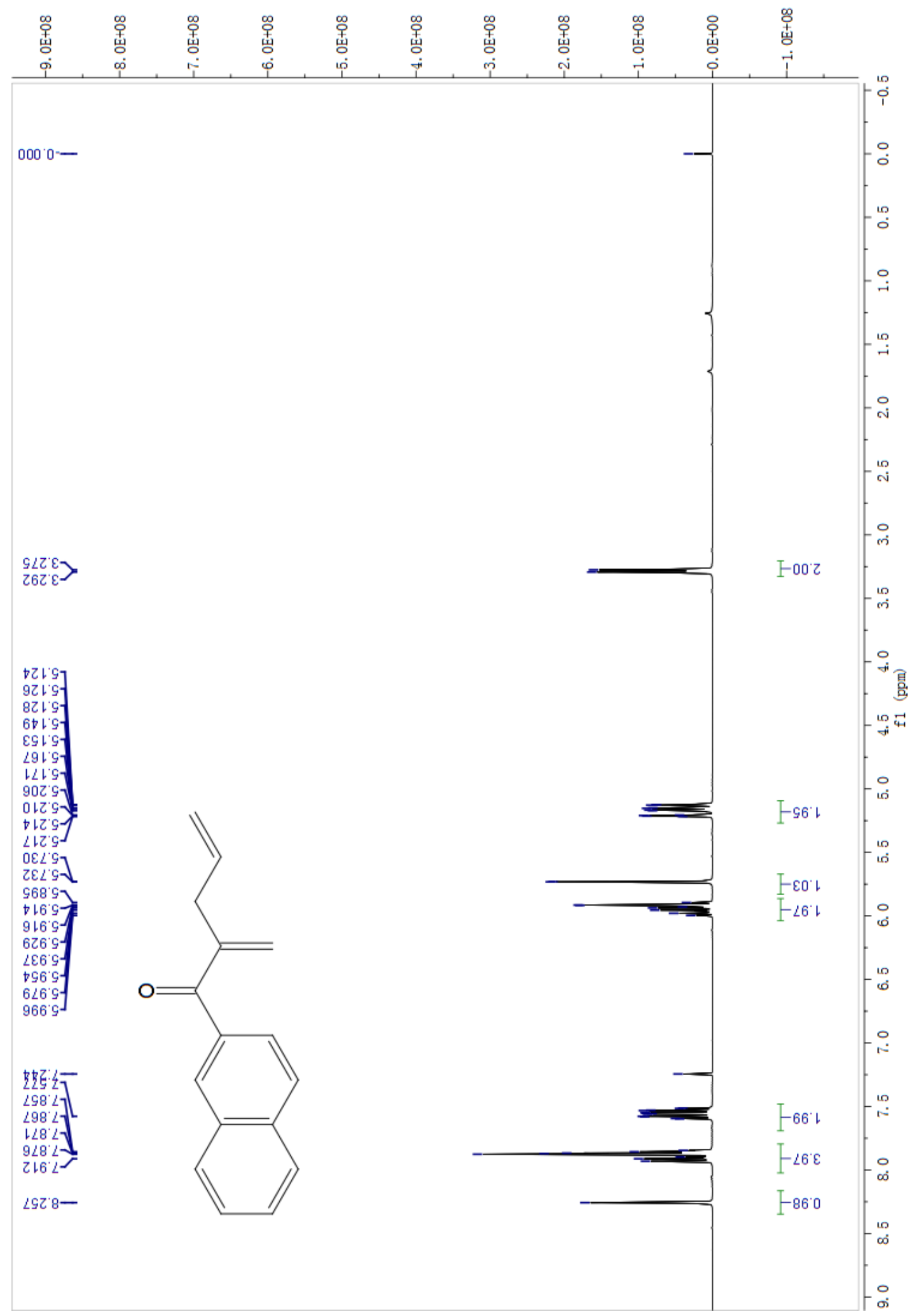


${ }^{13}$ C NMR Spectrum of 2-Methylene-1-(naphthalen-2-yl)pent-4-en-1-one 3ma

\begin{tabular}{|c|c|c|c|c|c|c|c|c|c|c|c|c|c|}
\hline $\begin{array}{l}\text { o } \\
\text { ㅇ } \\
\text { 녕 } \\
\varphi\end{array}$ & $\begin{array}{l}\text { 号 } \\
\text { 足 } \\
\text { 岇 } \\
\text { ம் }\end{array}$ & $\begin{array}{l}\text { o } \\
\text { + } \\
\text { 형 } \\
\text { ம }\end{array}$ & 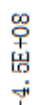 & $\begin{array}{l}\text { o } \\
\text { ㅇ } \\
\text { 봉 } \\
\dot{+}\end{array}$ & 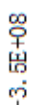 & $\begin{array}{l}\text { o } \\
\text { + } \\
\text { 넝 } \\
\text { ம }\end{array}$ & $\begin{array}{l}\infty \\
\text { o } \\
\text { 峁 } \\
\text { }\end{array}$ & $\begin{array}{l}\text { o } \\
\text { ㅇ } \\
\text { 병 } \\
\text { ㄱ }\end{array}$ & $\begin{array}{l}\infty \\
\text { 古 } \\
\text { 赑 } \\
-\end{array}$ & $\begin{array}{l}\text { o } \\
\text { + } \\
\text { 봉 } \\
-\end{array}$ & $\begin{array}{l}\text { 무 } \\
\text { 명 } \\
\text { 웅 }\end{array}$ & $\begin{array}{l}\text { 오 } \\
\text { ㅇ } \\
\text { 봉 } \\
\text { ㅇ }\end{array}$ & 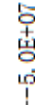 \\
\hline
\end{tabular}

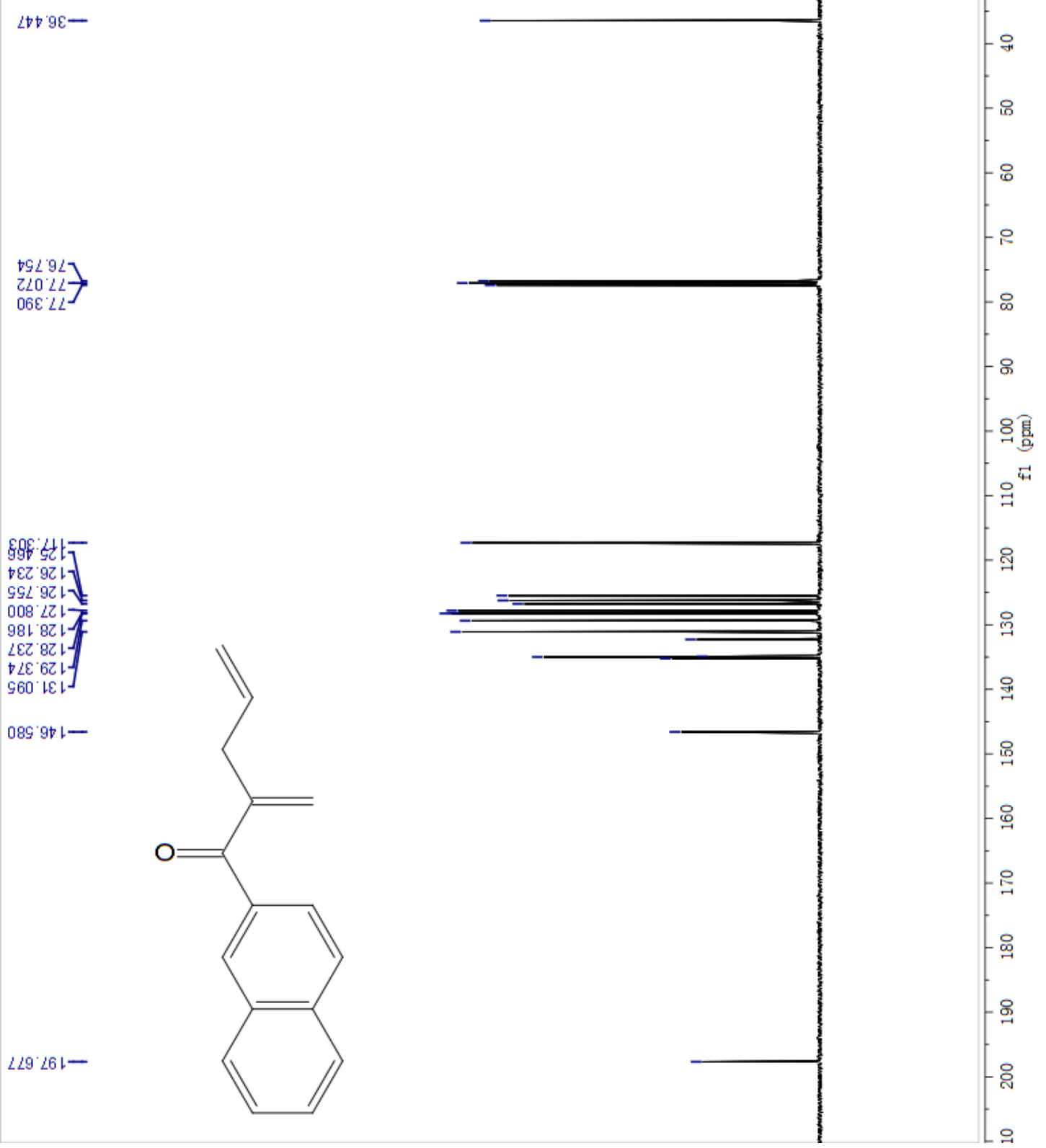


HR-MS Spectrum of 2-Methylene-1-(naphthalen-2-yl)pent-4-en-1-one 3ma

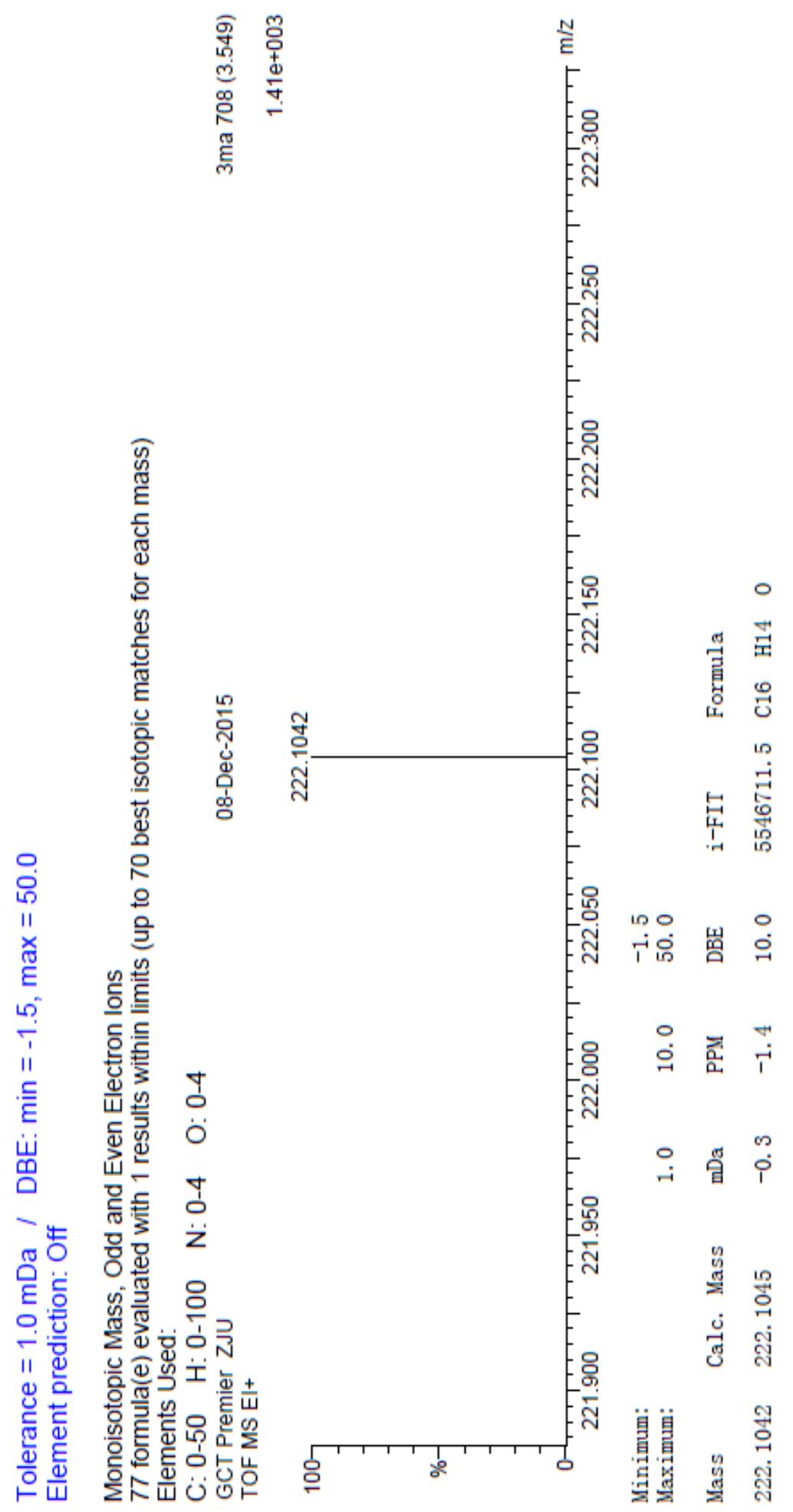


${ }^{1} \mathrm{H}$ NMR Spectrum of (E)-2-Methylene-5-phenyl-1-(4-tolyl)pent-4-en-1-one 3ab

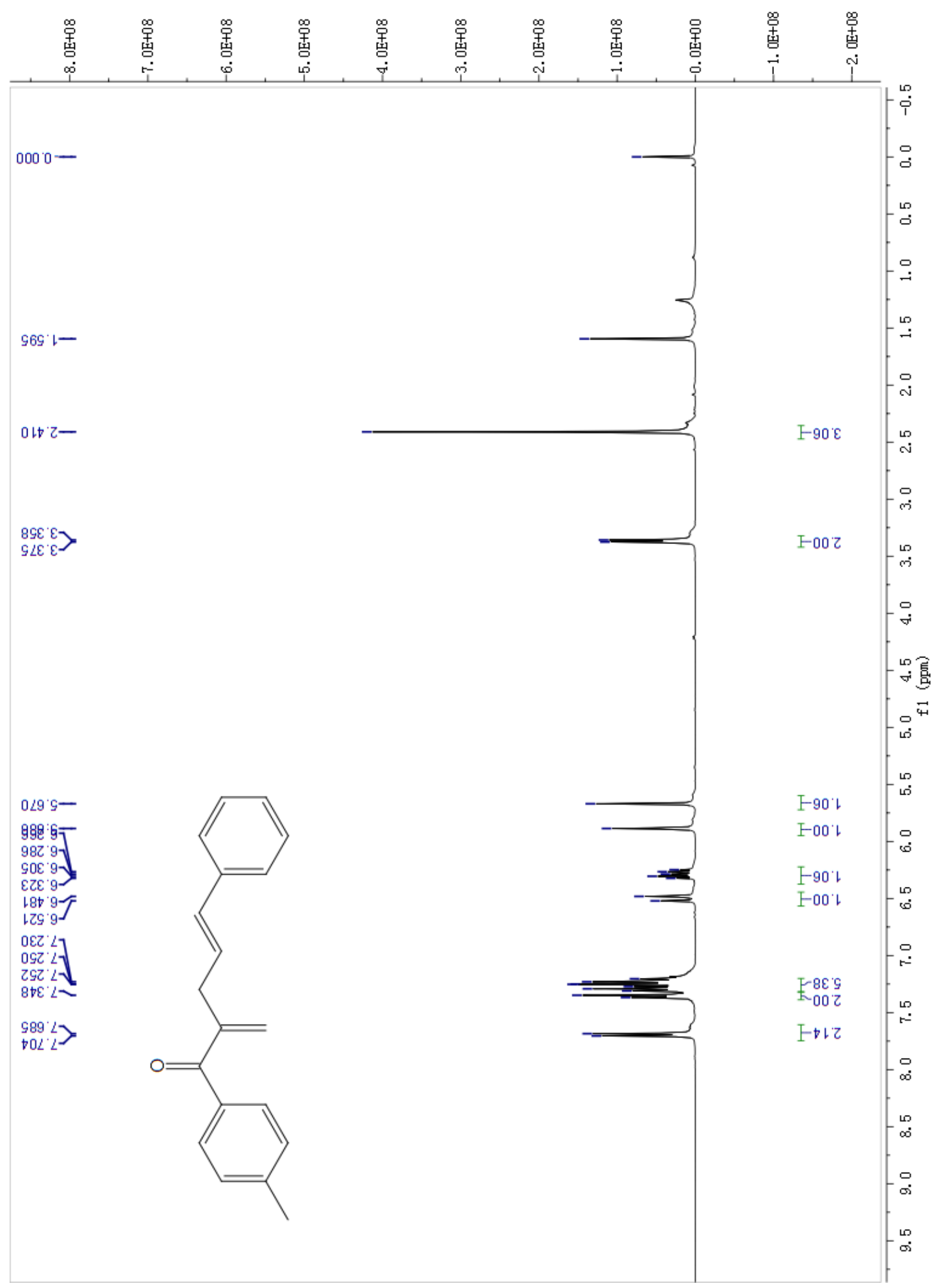


${ }^{13}$ C NMR Spectrum of (E)-2-Methylene-5-phenyl-1-(4-tolyl)pent-4-en-1-one 3ab

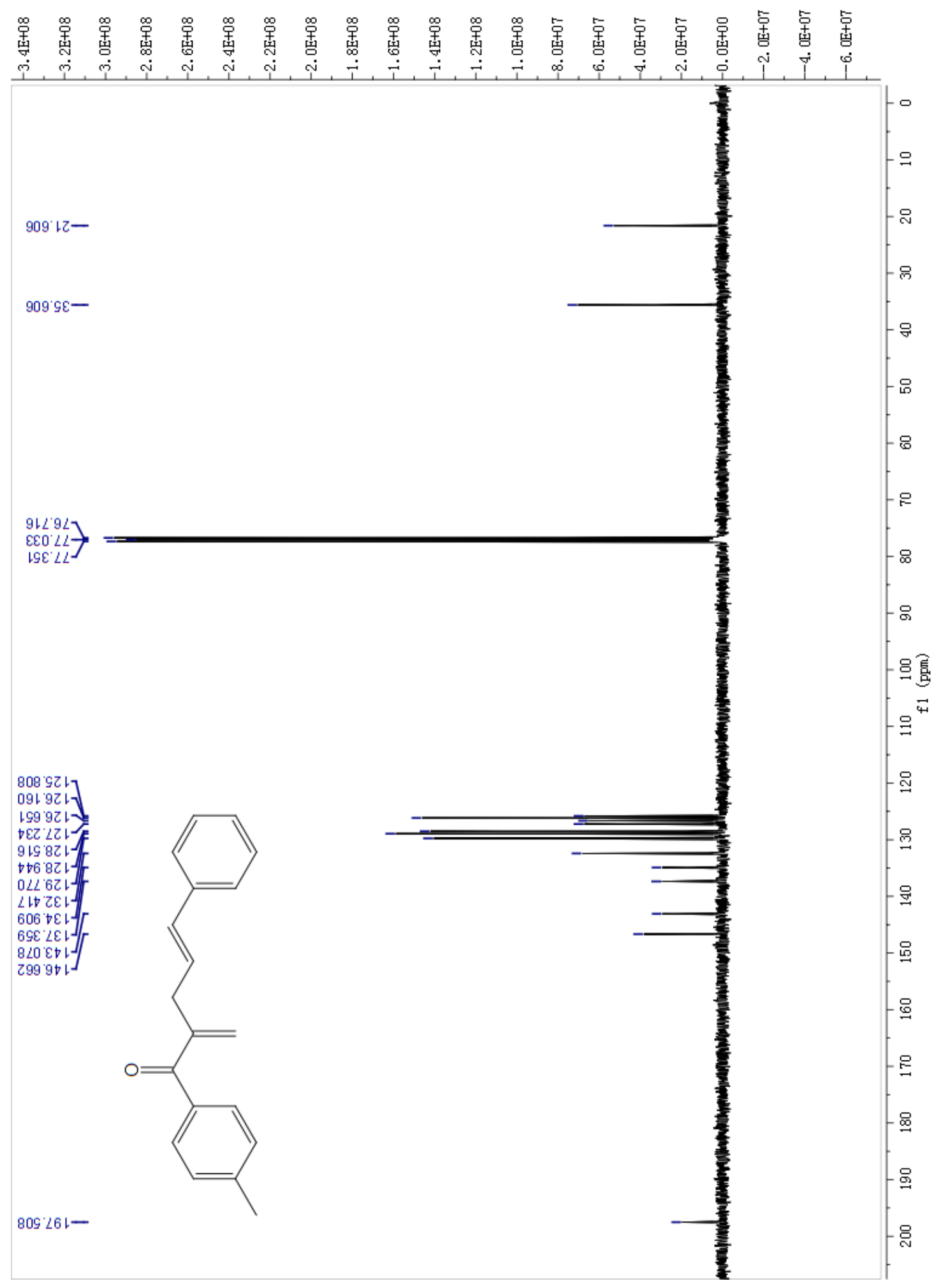


HR-MS Spectrum of (E)-2-Methylene-5-phenyl-1-(4-tolyl)pent-4-en-1-one 3ab

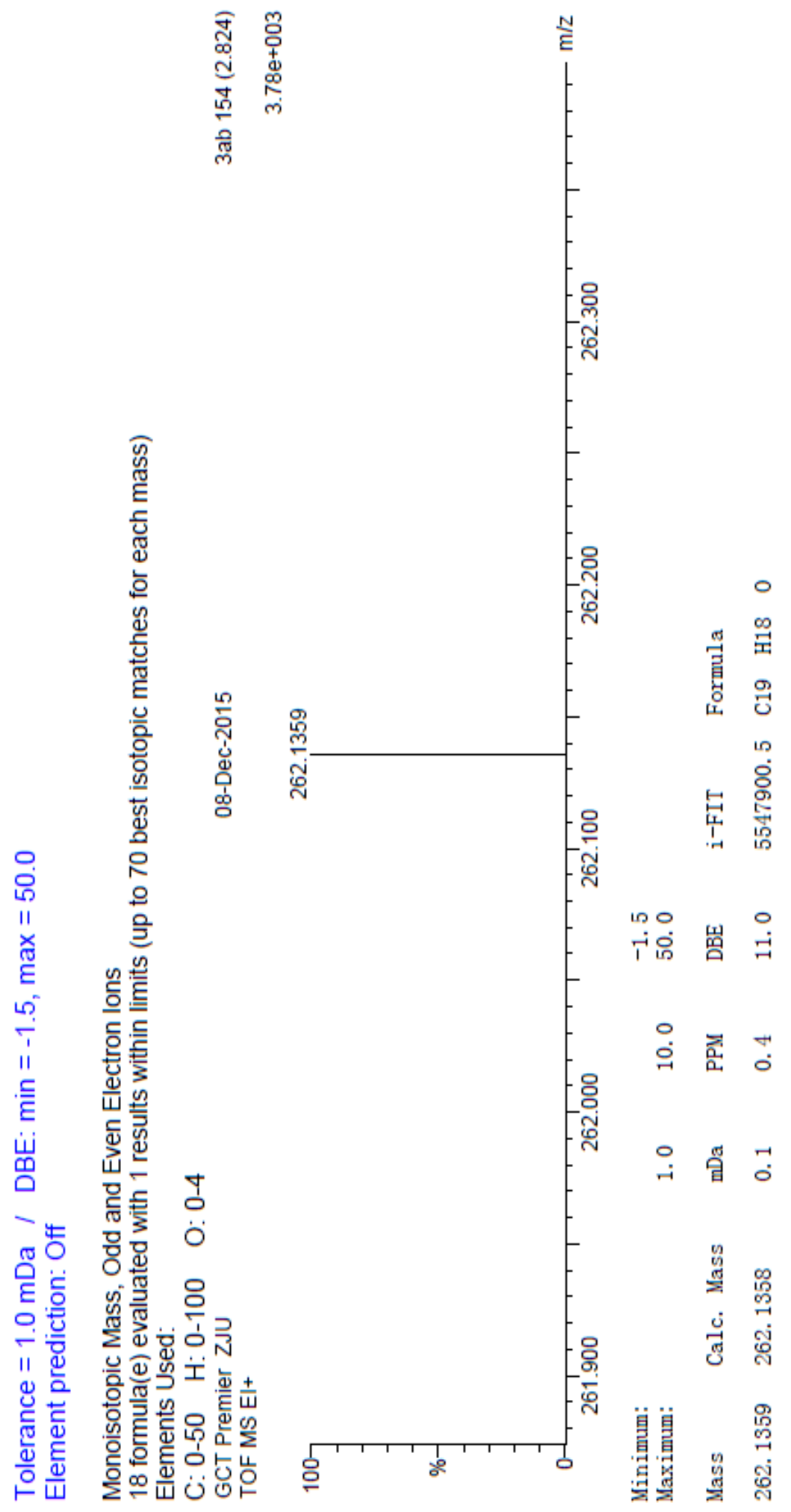


${ }^{1} \mathrm{H}$ NMR Spectrum of (E)-5-(2-Methoxyphenyl)-2-methylene-1-(4-tolyl)pent-4-en -1-one 3ac

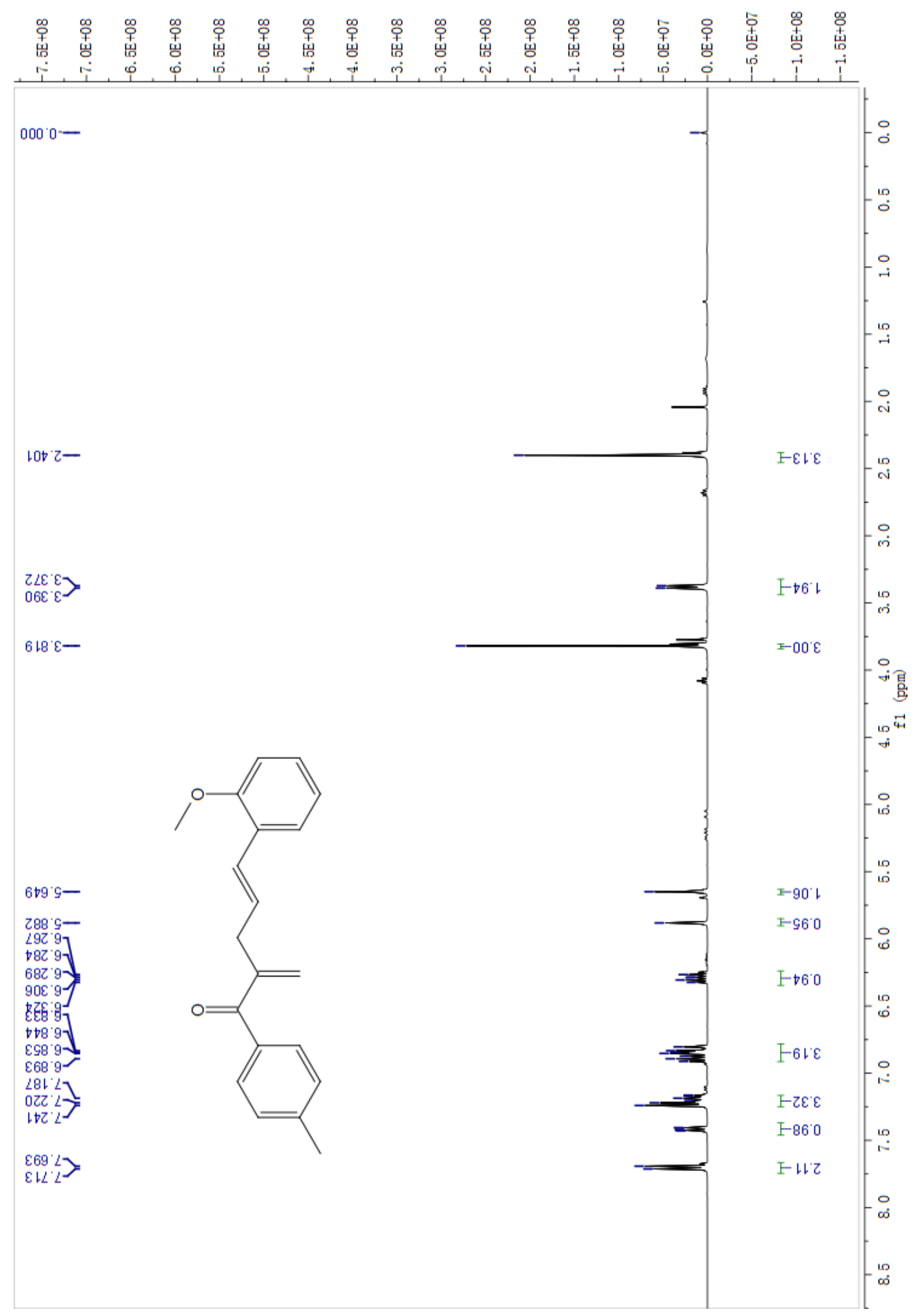


${ }^{13}$ C NMR Spectrum of (E)-5-(2-Methoxyphenyl)-2-methylene-1-(4-tolyl)pent-4-en -1-one 3ac

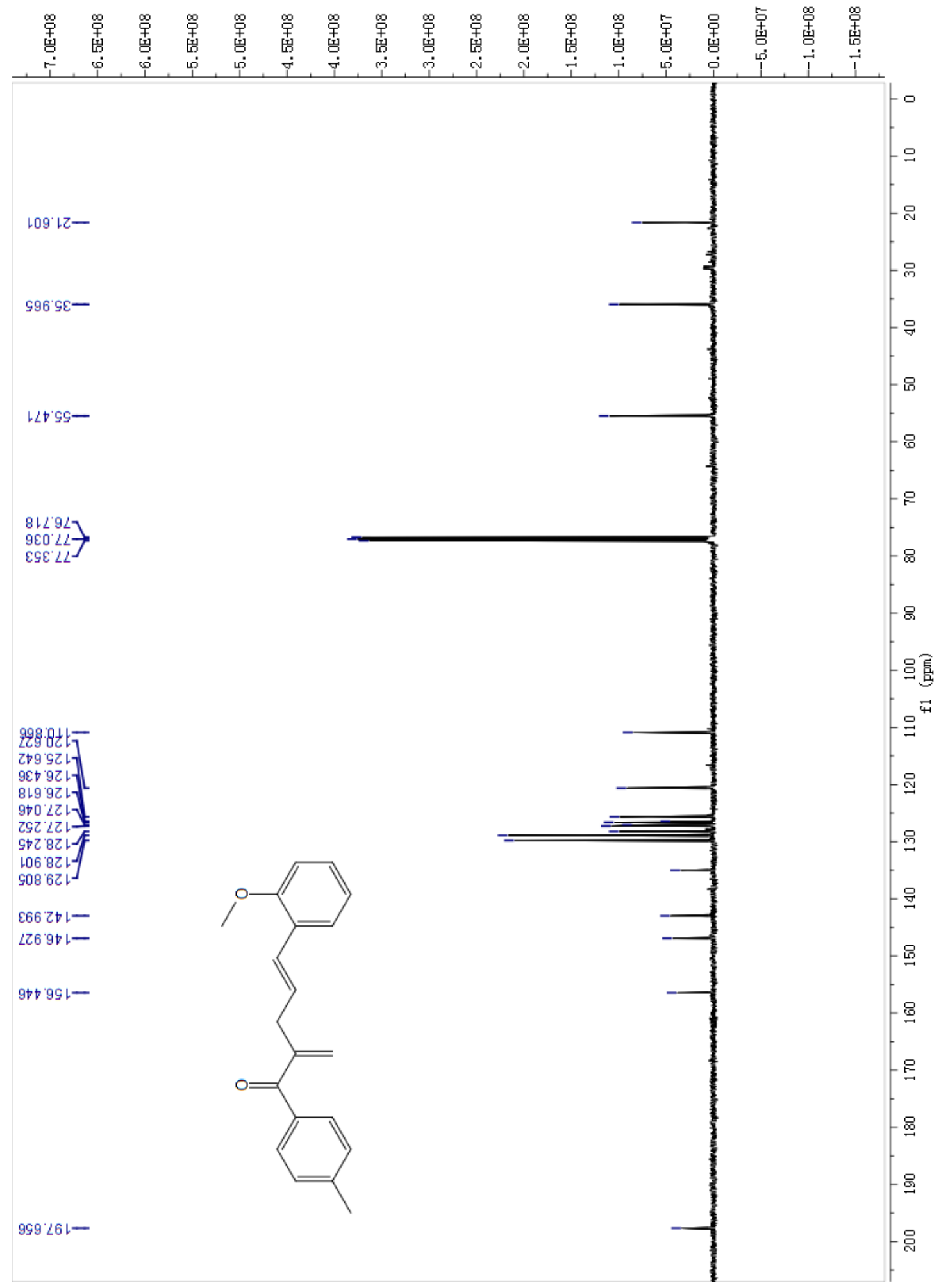


HR-MS Spectrum of (E)-5-(2-Methoxyphenyl)-2-methylene-1-(4-tolyl)pent-4-en -1-one 3ac

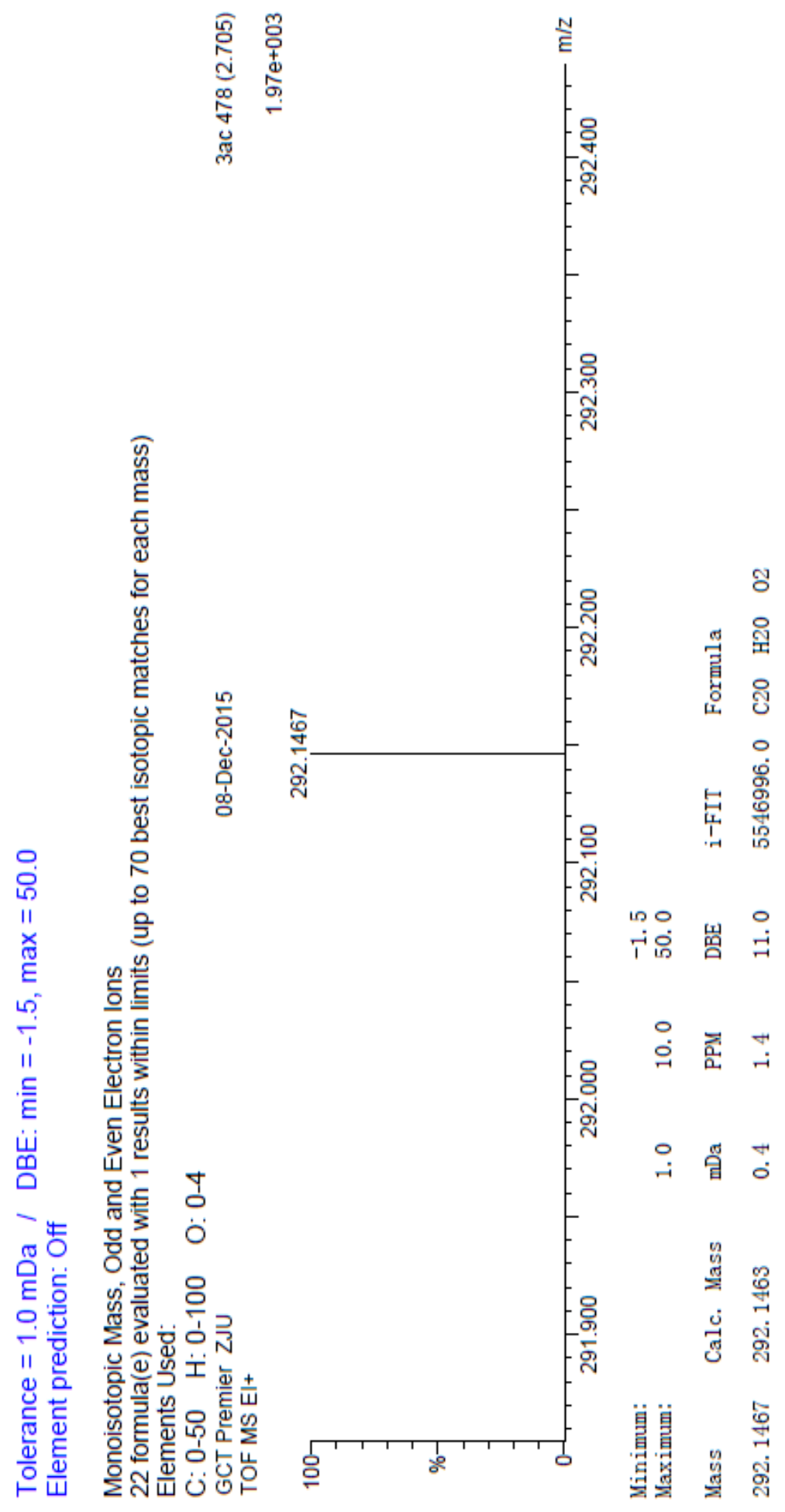


${ }^{1} \mathrm{H}$ NMR Spectrum of (E)-5-(4-Fluorophenyl)-2-methylene-1-(4-tolyl)pent-4-en-1-one 3ad

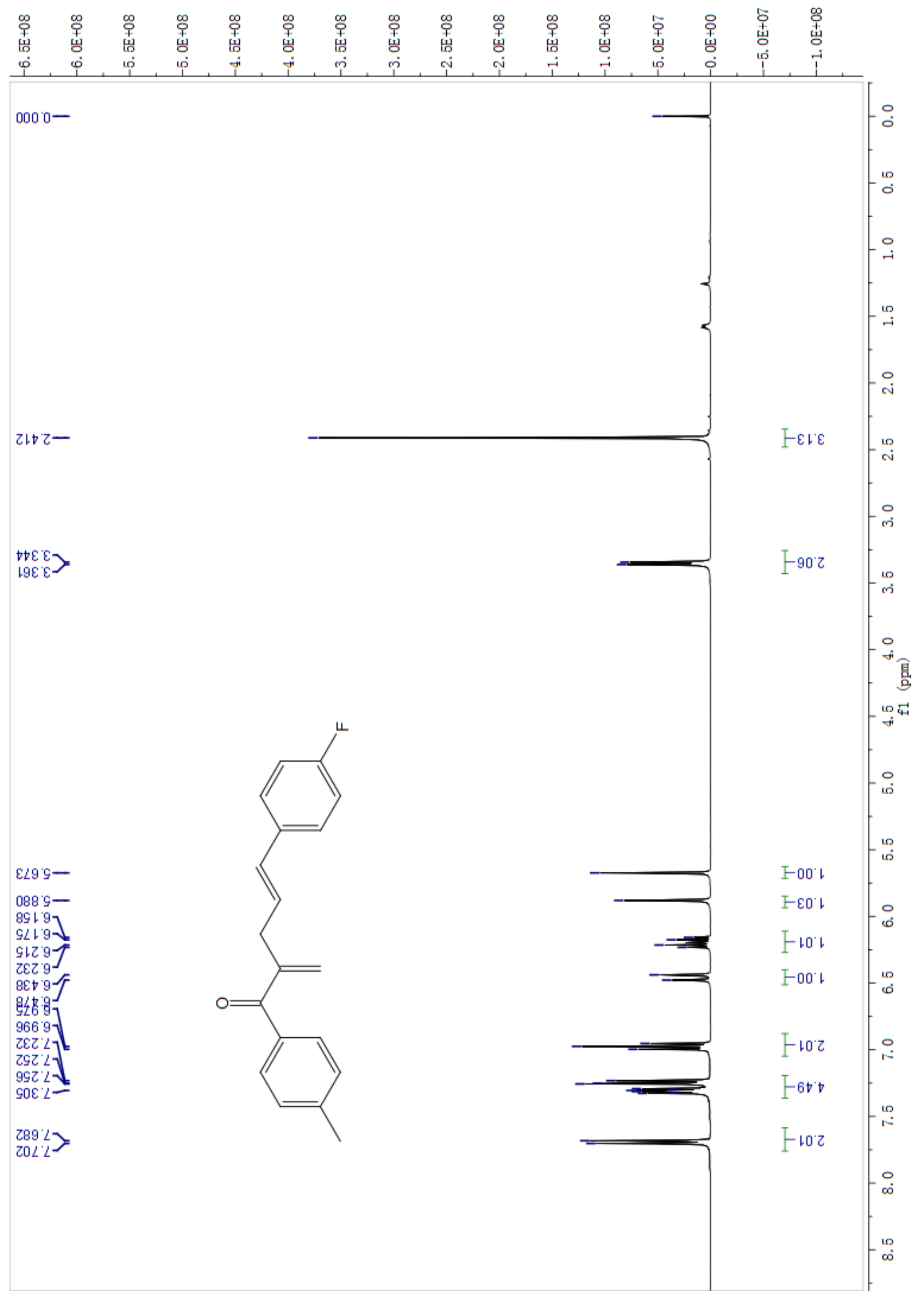


${ }^{13}$ C NMR Spectrum of (E)-5-(4-Fluorophenyl)-2-methylene-1-(4-tolyl)pent-4-en -1-one 3ad

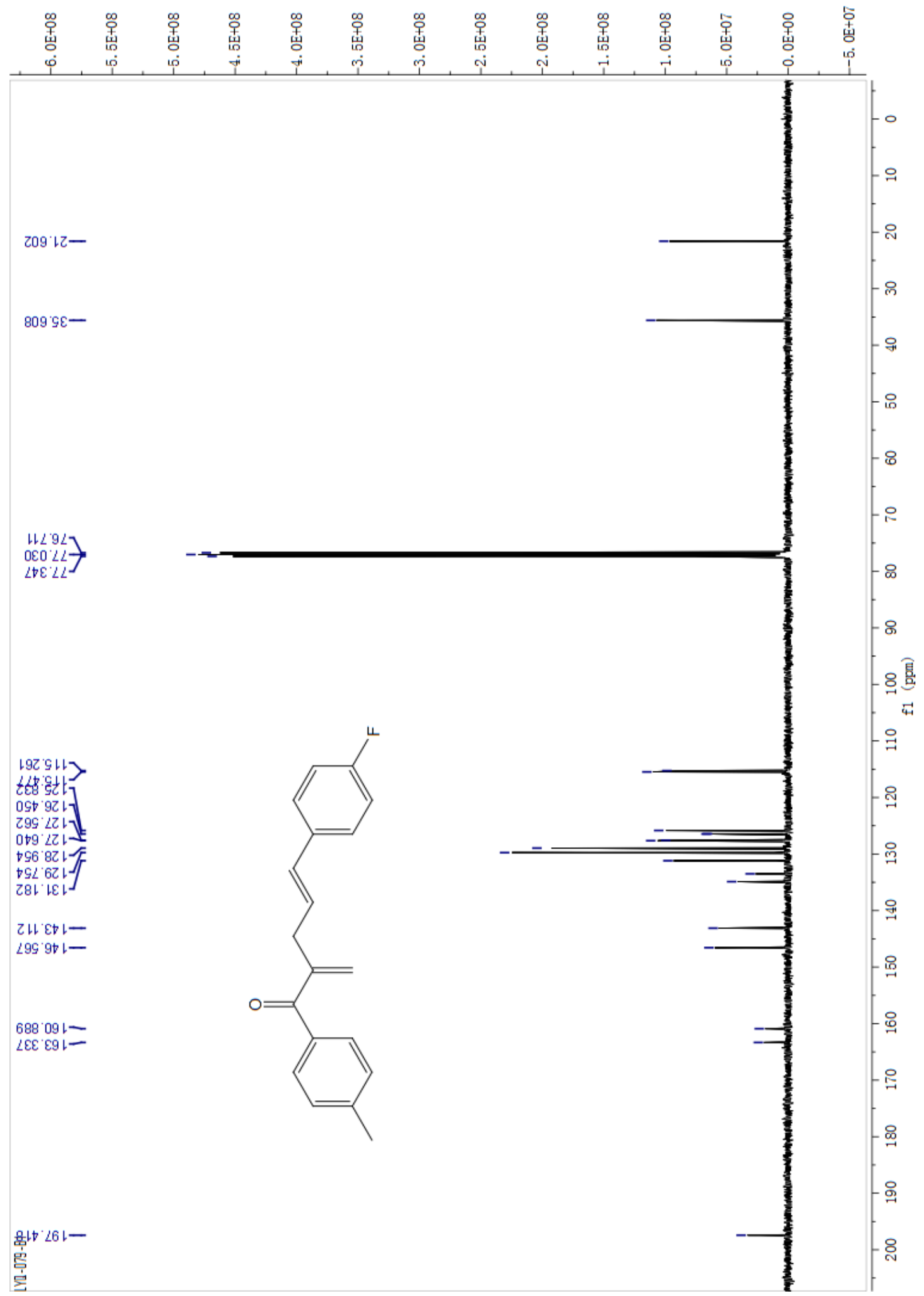


HR-MS Spectrum of (E)-5-(4-Fluorophenyl)-2-methylene-1-(4-tolyl)pent-4-en-1-one 3ad

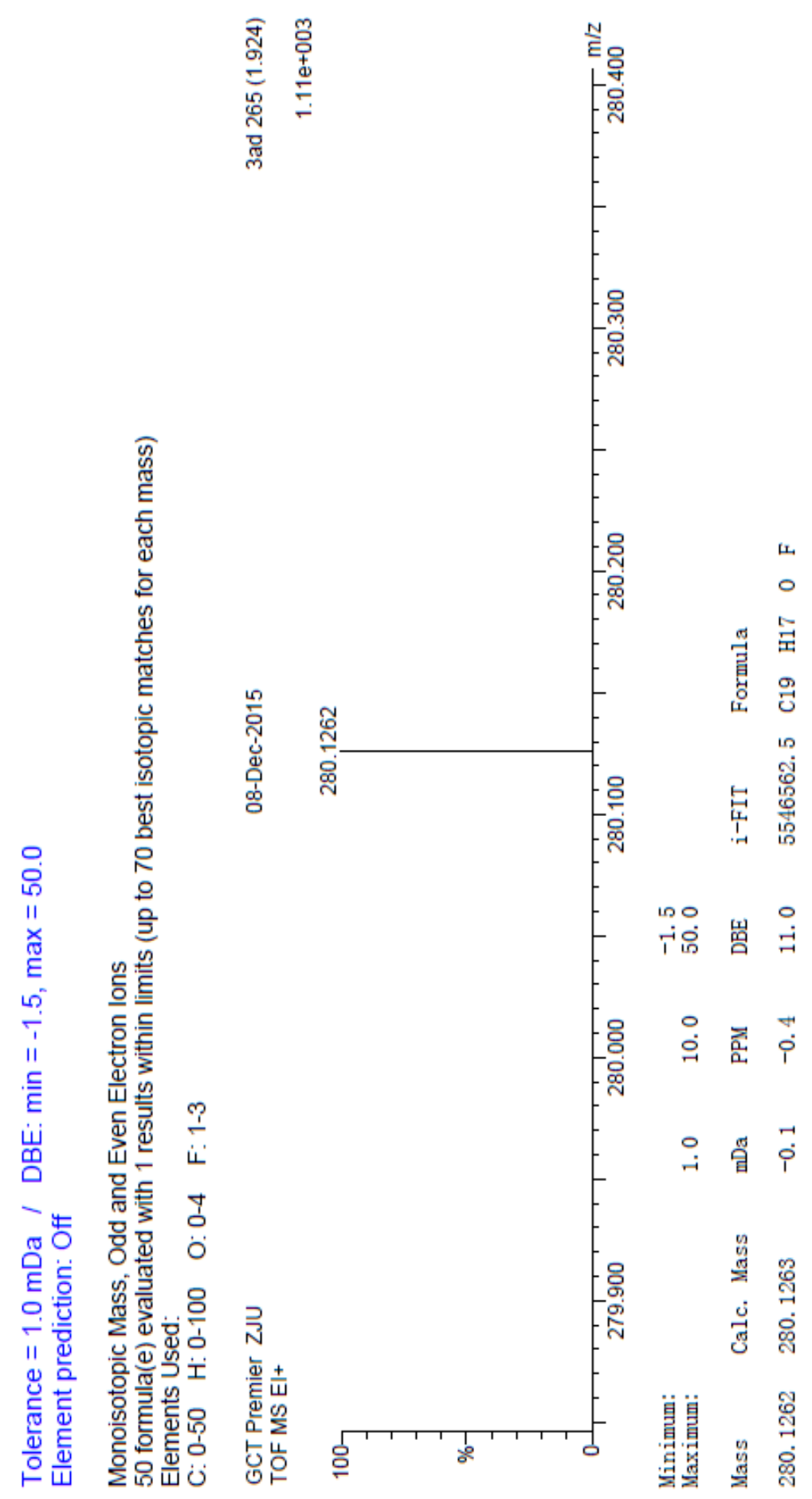


${ }^{1} \mathrm{H}$ NMR Spectrum of (E)-5-(4-Chlorophenyl)-2-methylene-1-(4-tolyl)pent-4-en -1-one 3ae

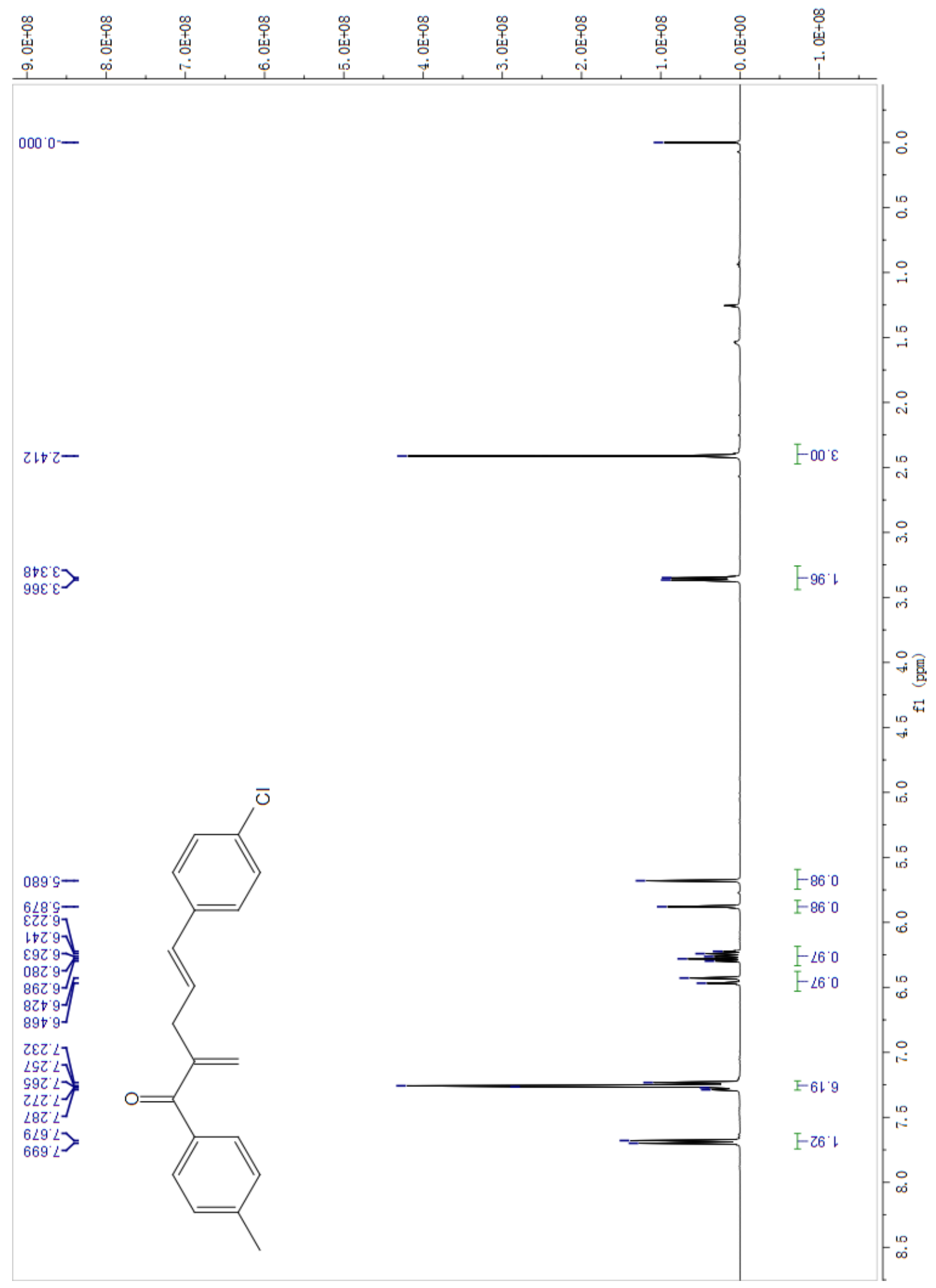


${ }^{13}$ C NMR Spectrum of (E)-5-(4-Chlorophenyl)-2-methylene-1-(4-tolyl)pent-4-en -1-one 3ae

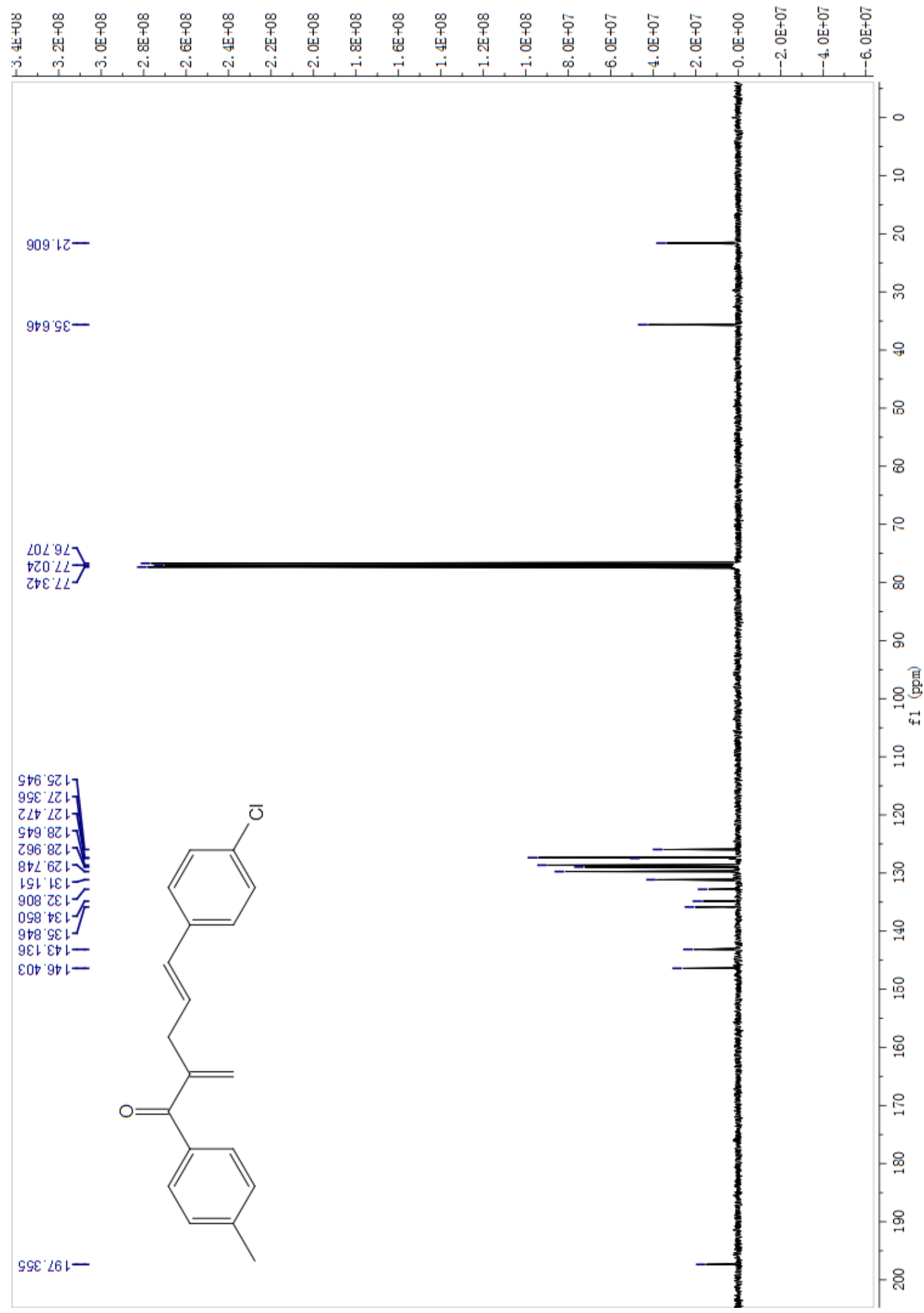


HR-MS Spectrum of (E)-5-(4-Chlorophenyl)-2-methylene-1-(4-tolyl)pent-4-en-1-one 3ae

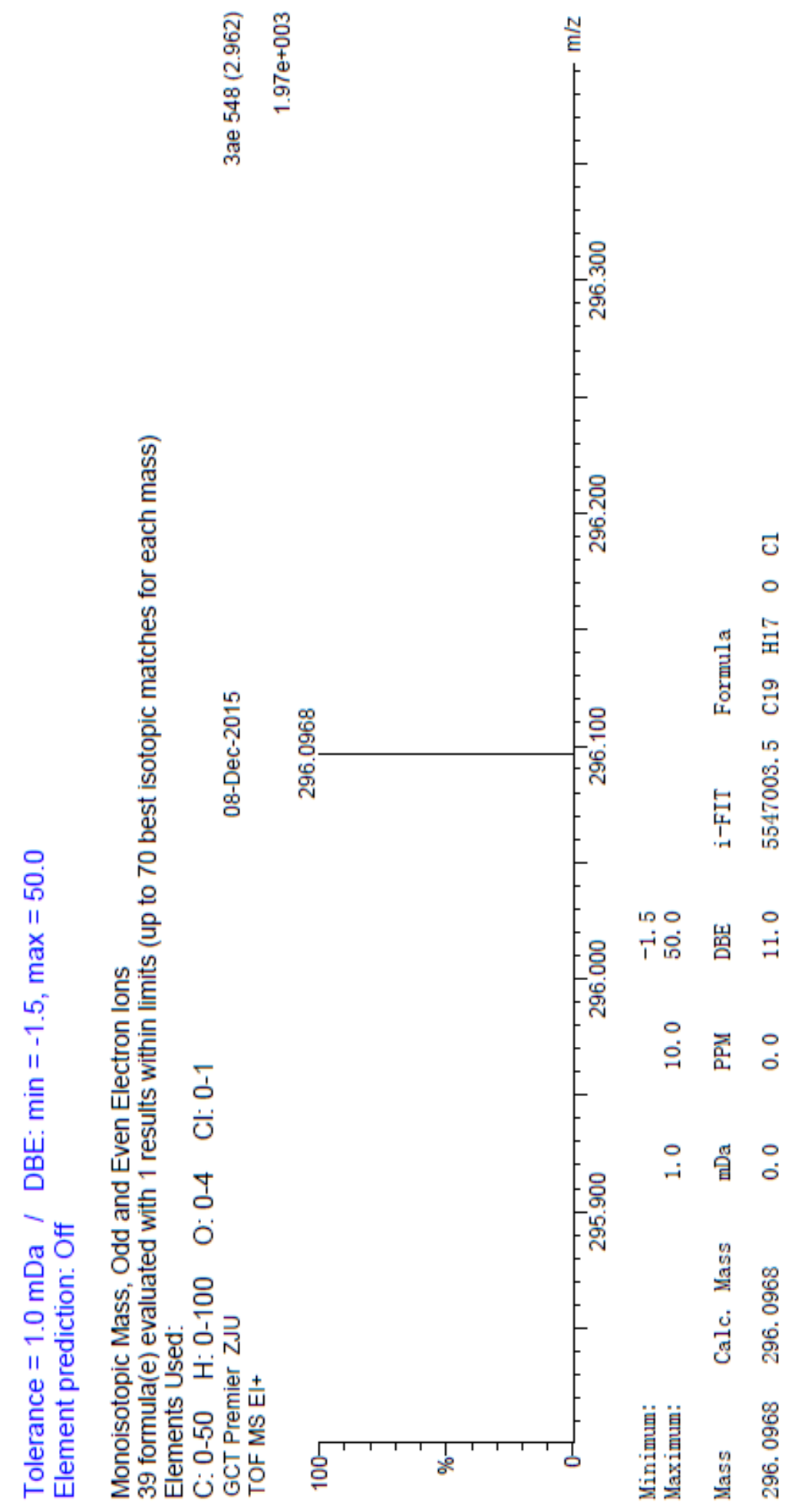


${ }^{1} \mathrm{H}$ NMR Spectrum of (E)-4-Methyl-2-methylene-5-phenyl-1-(4-tolyl)pent-4-en-1-one 3af

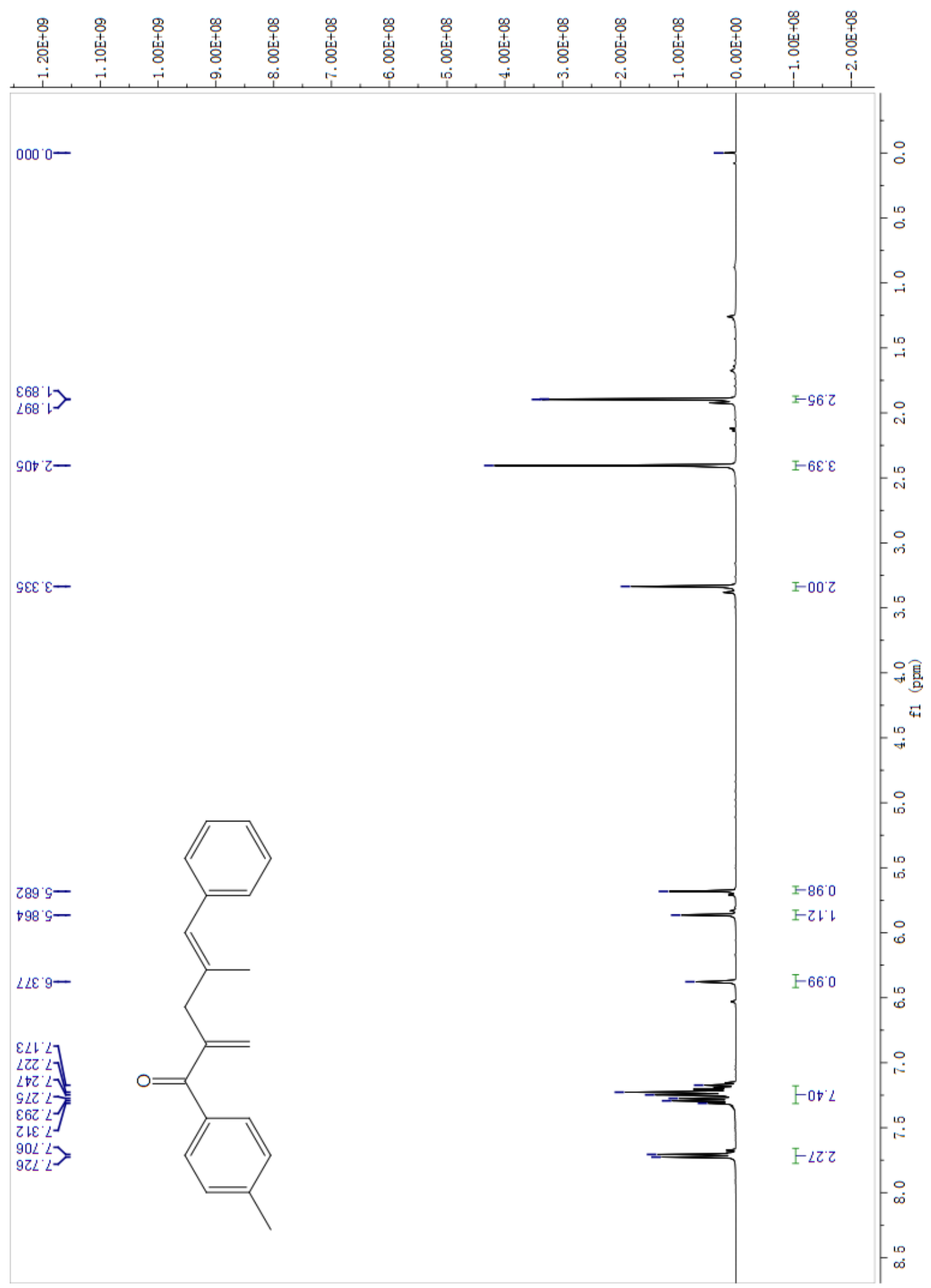


${ }^{13} \mathrm{C}$ NMR Spectrum of (E)-4-Methyl-2-methylene-5-phenyl-1-(4-tolyl)pent-4-en -1-one 3af

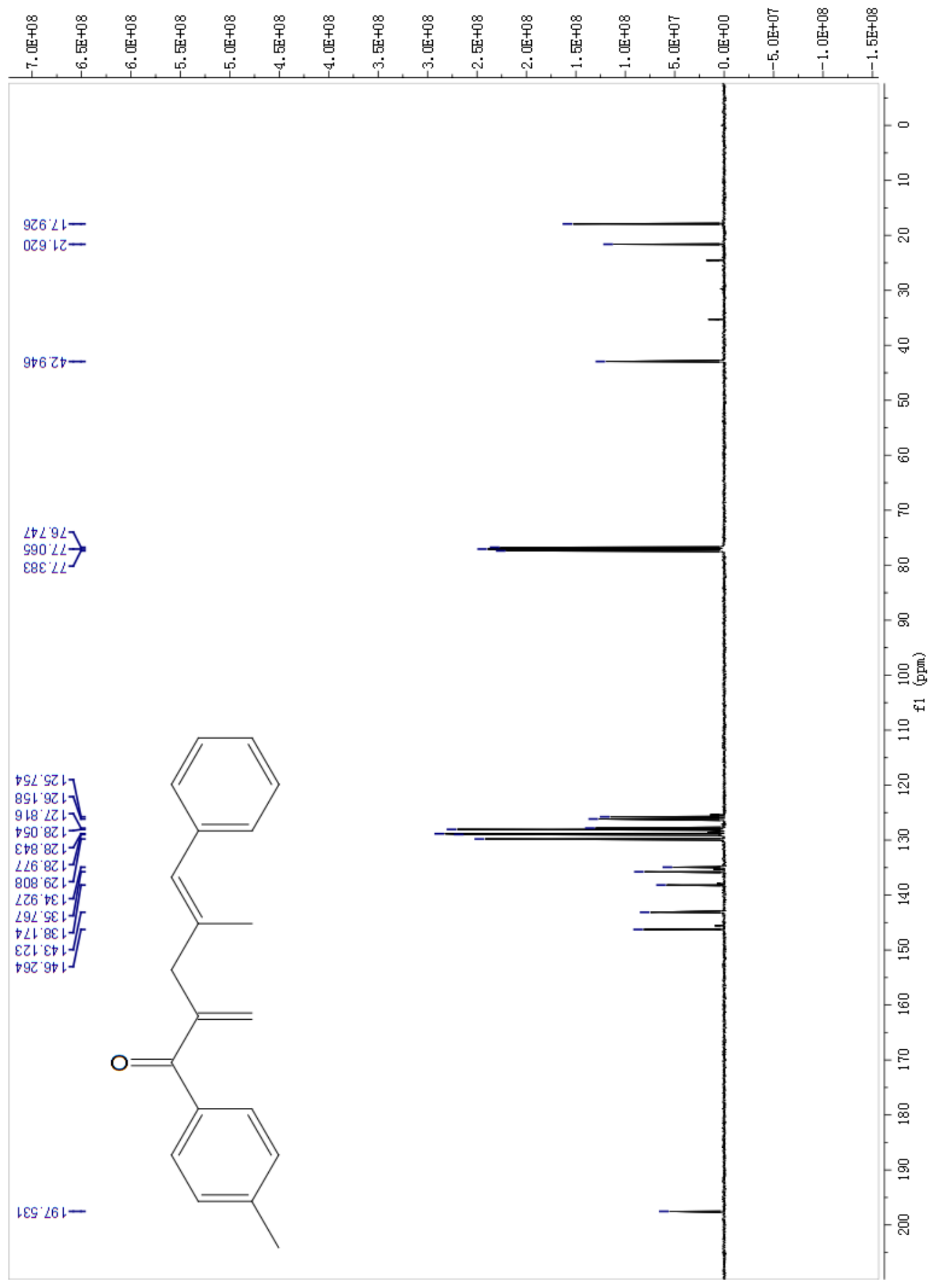


HR-MS Spectrum of (E)-4-Methyl-2-methylene-5-phenyl-1-(4-tolyl)pent-4-en-1-one 3af

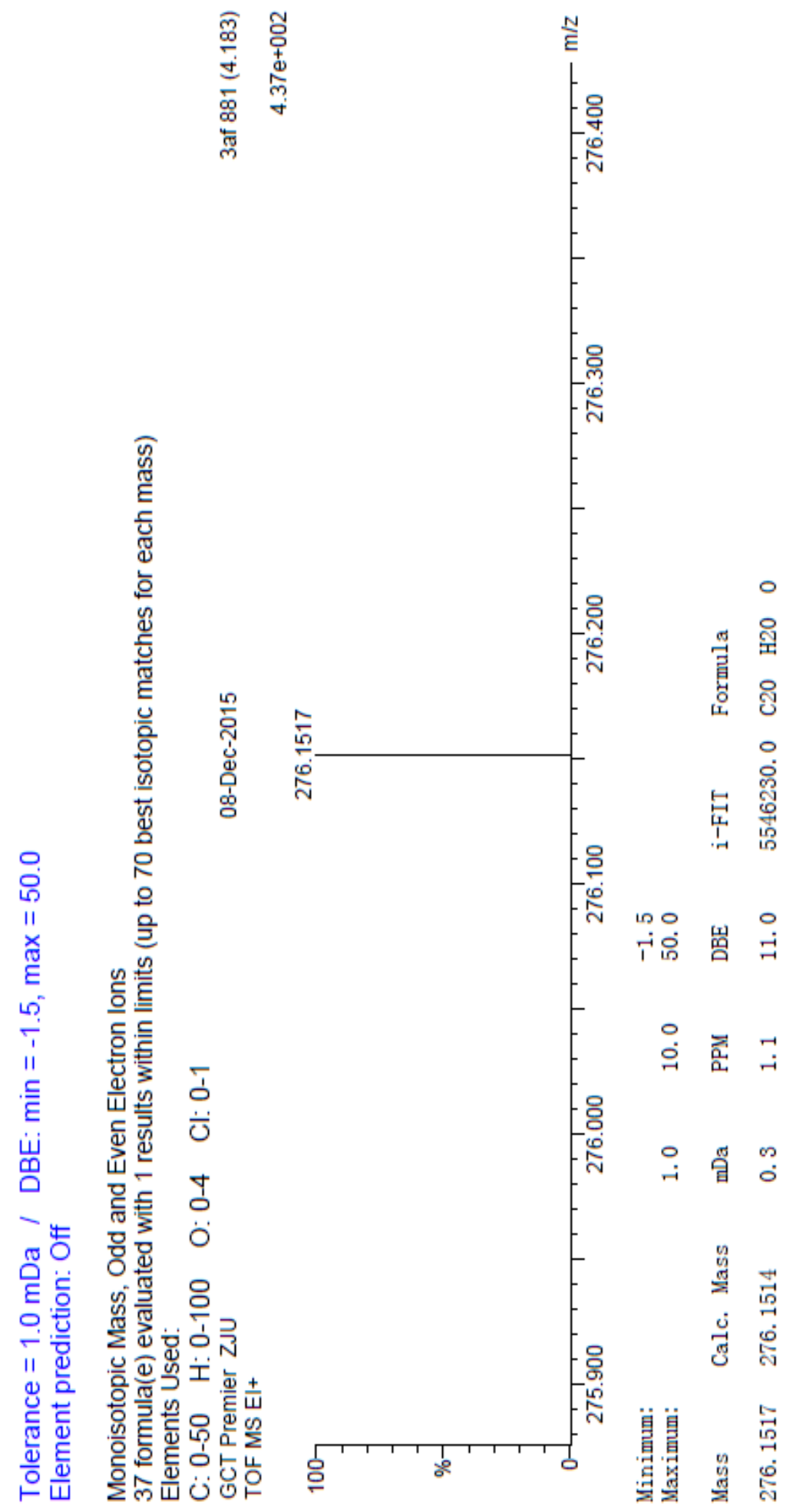


${ }^{1} \mathrm{H}$ NMR Spectrum of (E)-4-Methylene-7-phenylhept-6-en-3-one 3nb

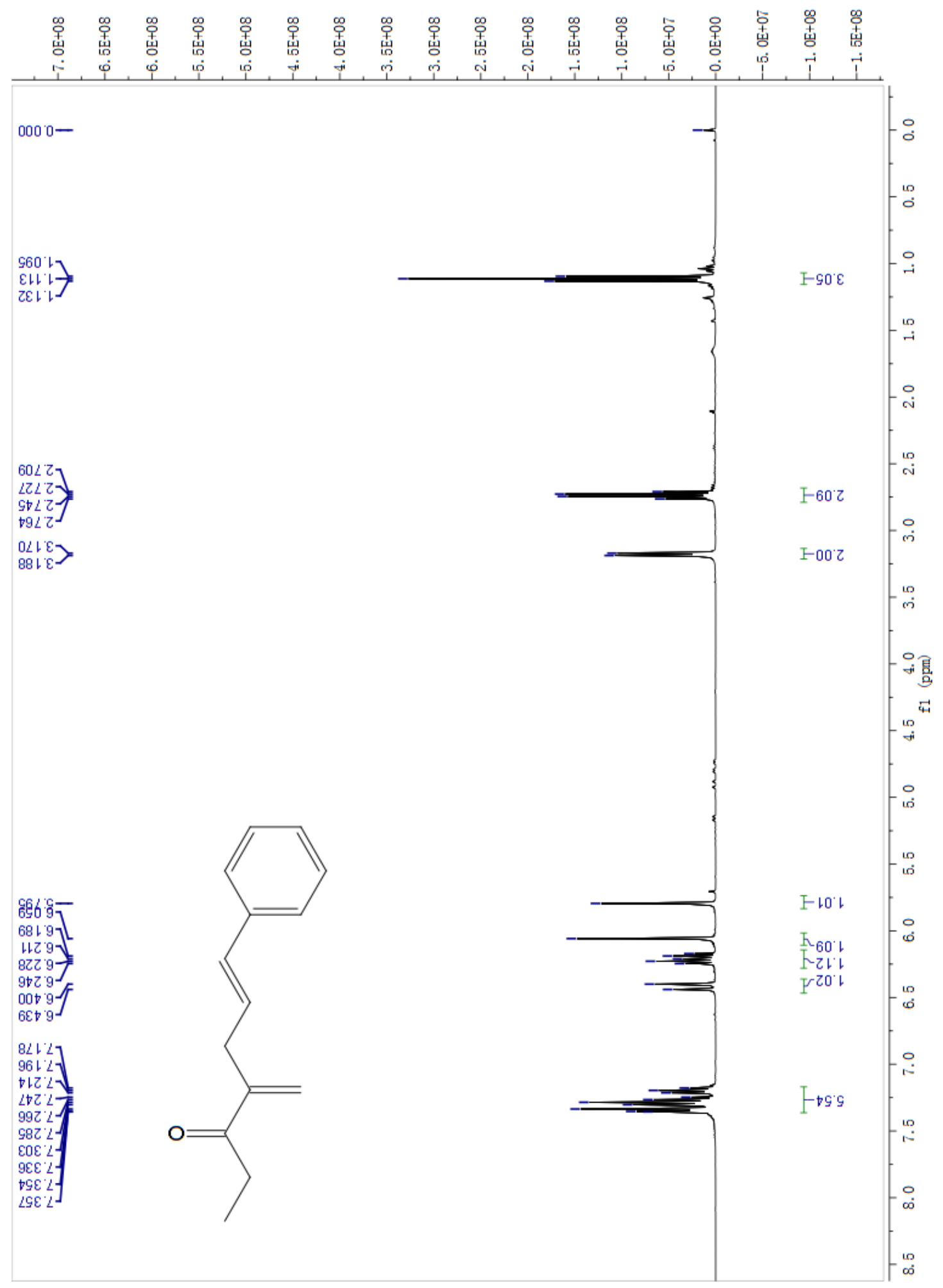


${ }^{13}$ C NMR Spectrum of (E)-4-Methylene-7-phenylhept-6-en-3-one 3nb

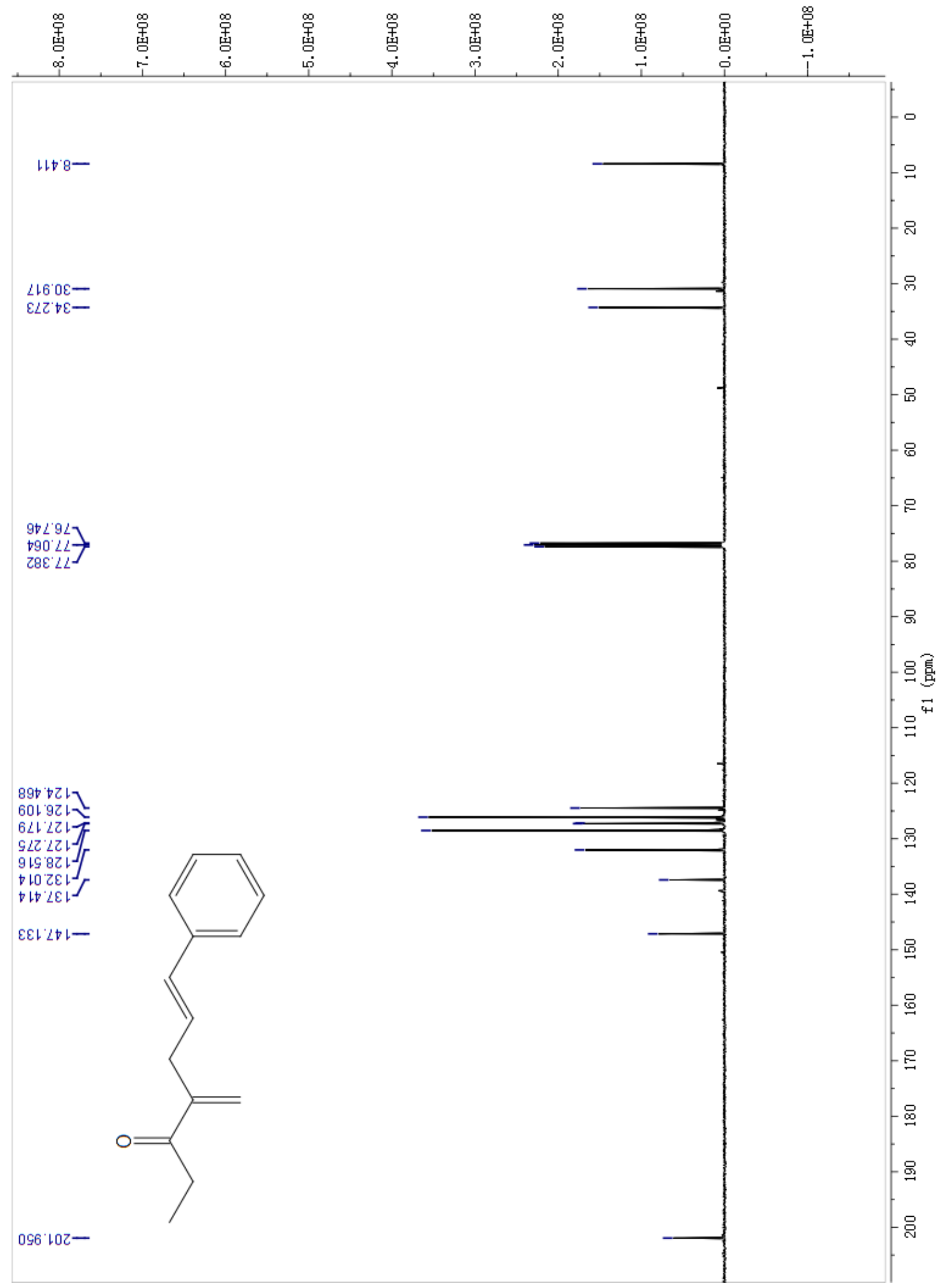


HR-MS Spectrum of (E)-4-Methylene-7-phenylhept-6-en-3-one 3nb

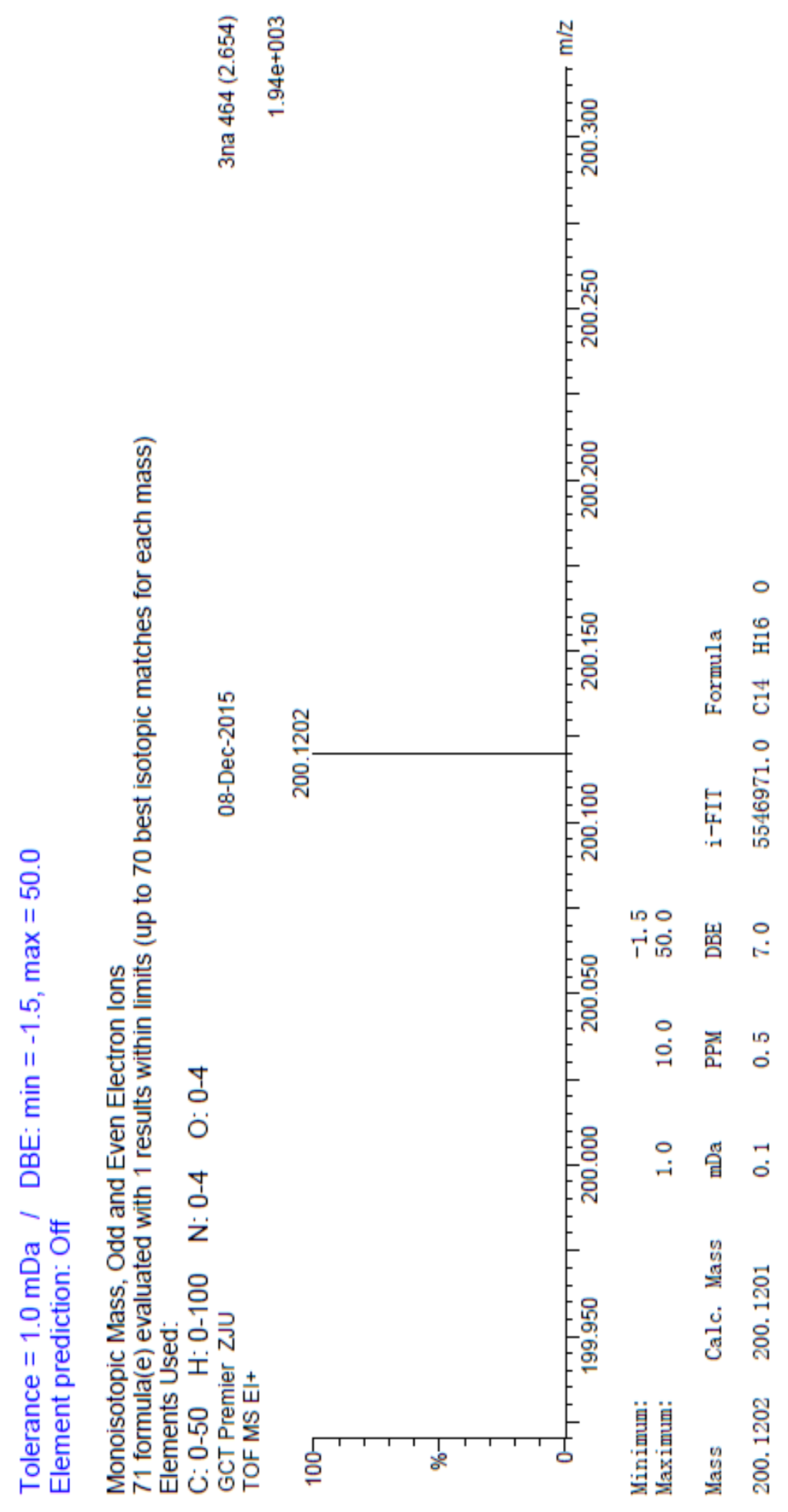


${ }^{1}$ H NMR Spectrum of Tributyl(3-oxo-3-(4-tolyl)propyl)phosphonium acetate 6

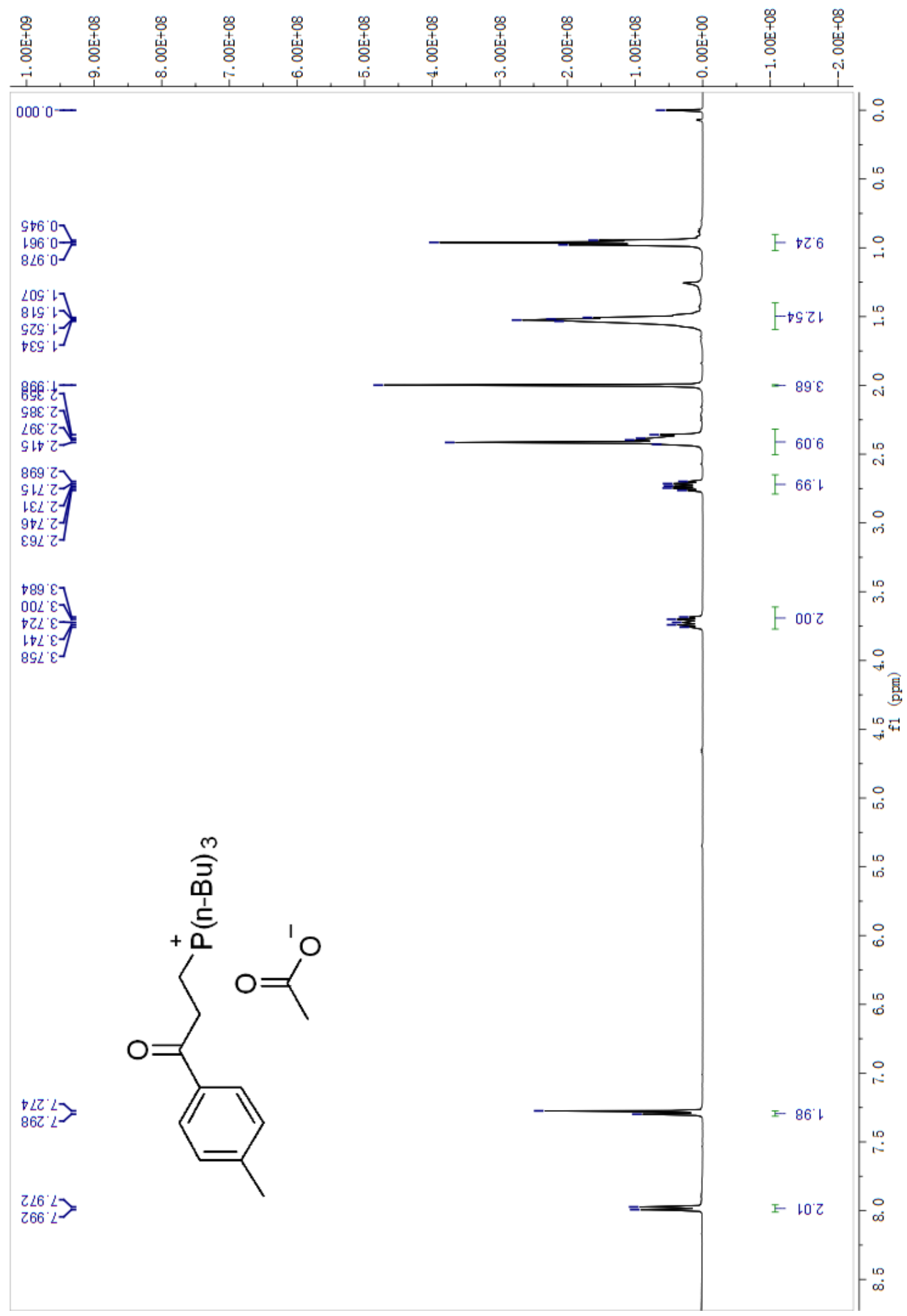


${ }^{13}$ C NMR Spectrum of Tributyl(3-oxo-3-(4-tolyl)propyl)phosphonium acetate 6

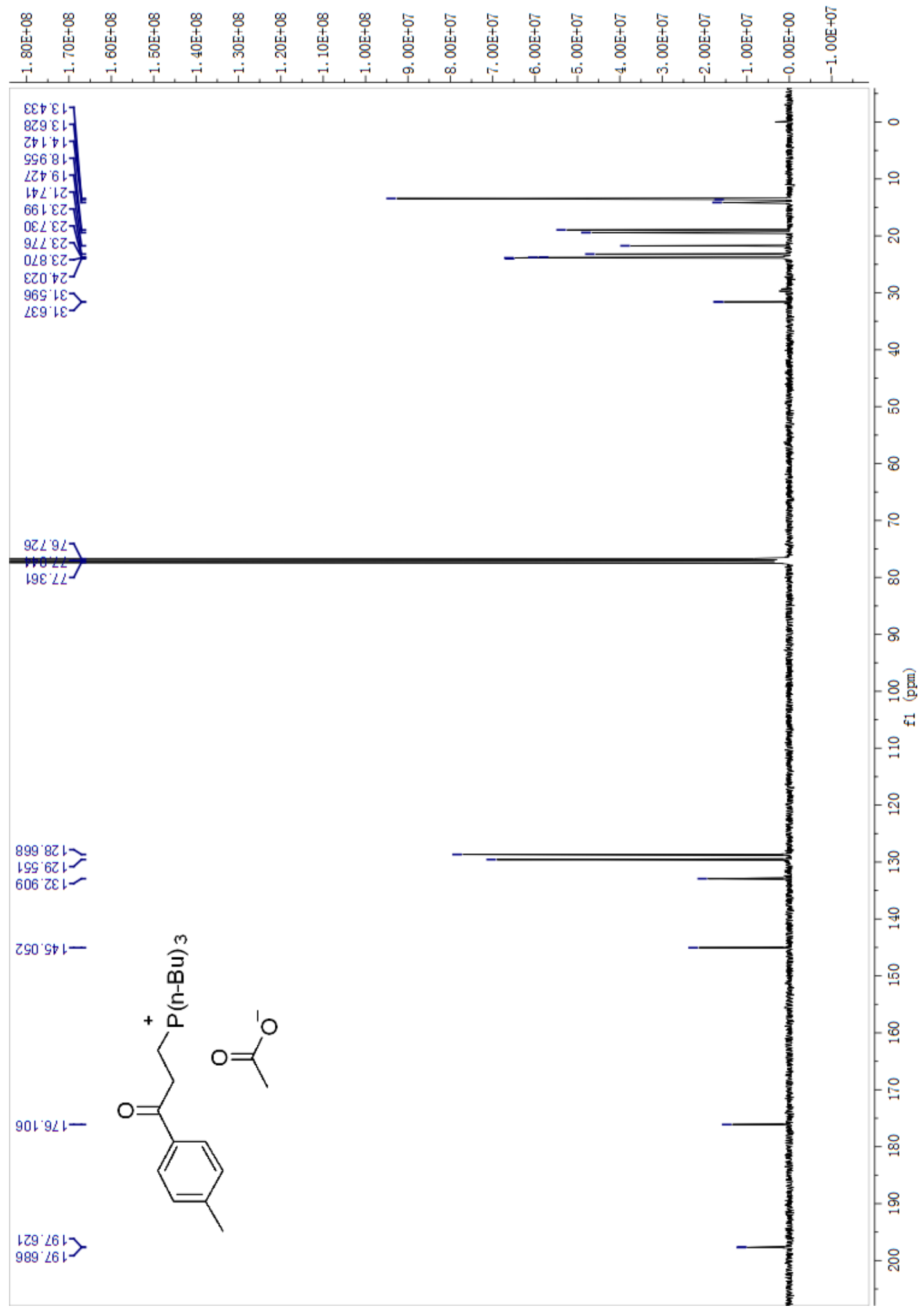


${ }^{31}$ P NMR Spectrum of Tributyl(3-oxo-3-(4-tolyl)propyl)phosphonium acetate 6

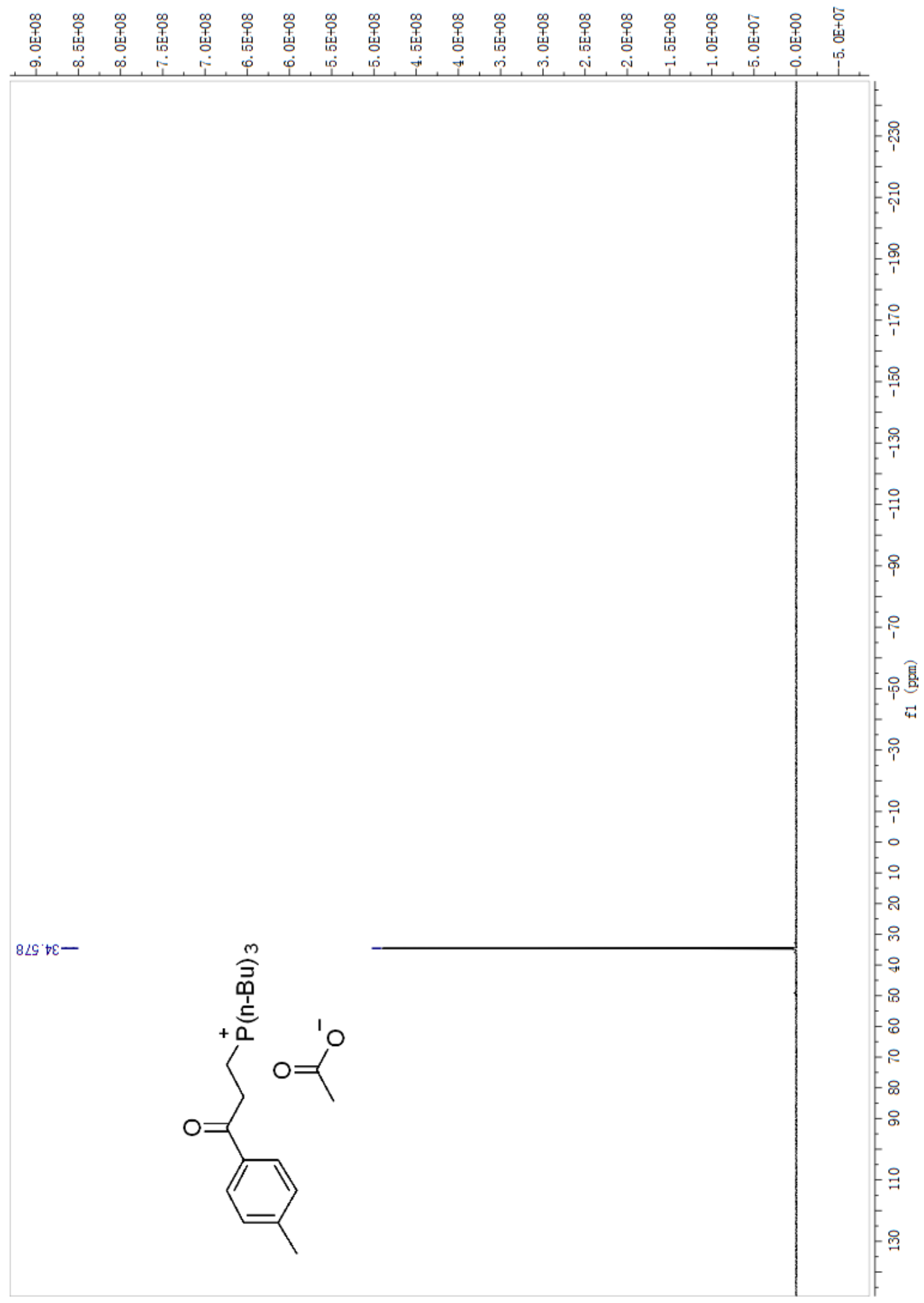


MS(ESI) Spectrum of Tributyl(3-oxo-3-(4-tolyl)propyl)phosphonium acetate 6

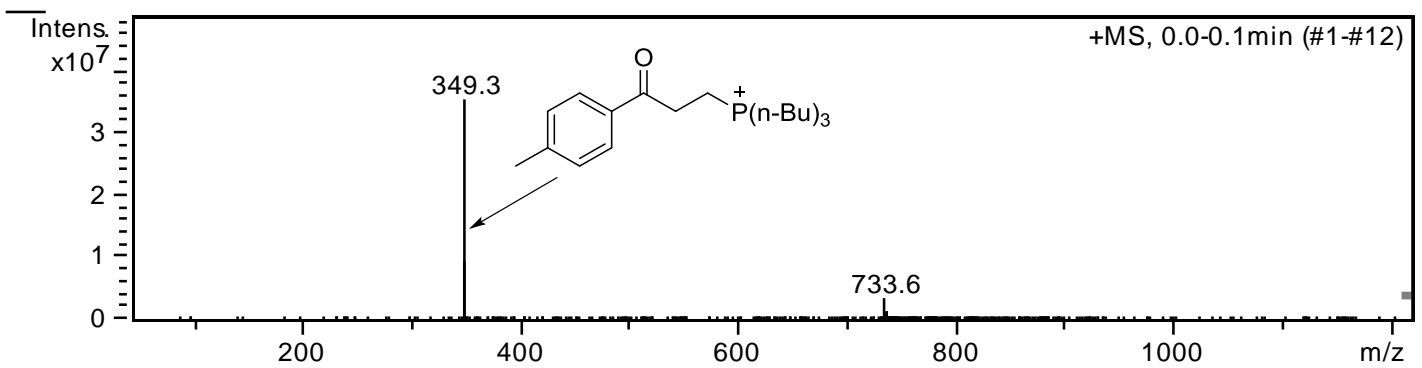

